\title{
THE EFFECTS OF OBESITY ON RESULTANT KNEE JOINT LOADS FOR GAIT AND CYCLING
}

\author{
A Thesis \\ presented to \\ the Faculty of California Polytechnic State University, \\ San Luis Obispo
}

In Partial Fulfillment

of the Requirements for the Degree

Master of Science in Mechanical Engineering

by

Juan David Gutierrez-Franco

June 2016 
C2016

Juan David Gutierrez-Franco

ALL RIGHTS RESERVED 
COMMITTEE MEMBERSHIP

TITLE: The Effects Of Obesity On Resultant Knee Joint Loads For Gait And Cycling

AUTHOR: Juan David Gutierrez-Franco

DATE SUBMITTED: June 2016

COMMITTEE CHAIR: Hemanth Porumamilla, Ph.D.

Associate Professor of Mechanical Engineering

COMMITTEE MEMBER: Stephen Klisch, Ph.D.

Professor of Mechanical Engineering

COMMITTEE MEMBER: Scott Hazelwood, Ph.D.

Professor of Biomedical Engineering 


\section{ABSTRACT \\ The Effects Of Obesity On Resultant Knee Joint Loads For Gait And Cycling Juan David Gutierrez-Franco}

Osteoarthritis $(\mathrm{OA})$ is a degenerative disease of cartilage and bone tissue and the most common form of arthritis, accounting for US\$10.5 billion in hospital charges in 2006. Obesity (OB) has been linked to increased risk of developing knee OA due to increased knee joint loads and varus-valgus misalignment. Walking is recommended as a weightloss activity but it may increase risk of knee $\mathrm{OA}$ as $\mathrm{OB}$ gait increases knee loads. Cycling has been proposed as an alternative weight-loss measure, however, lack of studies comparing normal weight (NW) and OB subjects in cycling and gait hinder identification of exercises that may best prevent knee OA incidence. The objective of this work is to determine if cycling is a better weight-loss exercise than gait in OB subjects as it relates to knee OA risk reduction due to decreased knee loads. A stationary bicycle was modified to measure forces and moments at the pedals in three dimensions. A pilot experiment was performed to calculate resultant knee loads during gait and cycling for NW $(n=4)$ and OB $(n=4)$ subjects. Statistical analyses were performed to compare knee loads and knee angles, and to determine statistical significance of results $(p<0.05)$. Cycling knee loads were lower than gait knee loads for all subjects $(p<0.033)$. OB axial knee loads were higher than NW axial knee loads in gait $(p=0.004)$ due to the weight-bearing nature of gait. No differences were observed in cycling knee loads between NW and OB subjects, suggesting cycling returns $\mathrm{OB}$ knee loads and biomechanics to normal levels. The lack of significant results in cycling could be due to the small sample size used or because rider weight is supported by the seat. Limitations to this study include small sample size, soft tissue artifact, and experimental errors in marker placement. Future studies should correct these limitations and find knee joint contact force rather than knee resultant loads using 
EMG-driven experiments. In conclusion, cycling loads were lower than gait loads for NW and OB subjects suggesting cycling is a better weight-loss exercise than gait in the context of reducing knee OA risk.

Keywords: Cycling, gait, obesity, knee loading, internal joint loads, biomechanics, motion capture 


\section{ACKNOWLEDGMENTS}

Thanks to Dr. Stephen Klisch and Dr. Scott Hazelwood for the mentorship and opportunities given to me during the last four years. The research experience at UCSD helped me grow as a scientist and the experience developing the HMB Lab at Cal Poly has made a better engineer.

Thanks to Dr. Hemanth Porumamilla for the mentorship and support in the project. The advice I received from him will help me in future projects and in my personal life.

Thanks to my lab mates, past and current, for helping with the project and for sharing in the frustrations this project brought. Students in the Human Motion Biomechanics lab played an important role in the development of this study. Luke Kramer assisted in the design and machining of the pedal assembly. Karim Dudum and Jake Deschamps installed the Motion Analysis system and helped developed gait experimental protocols. Eshan Dandekar provided kinesiology knowledge to develop cycling experimental protocols and aided in marker set placement. Jim Darke, Alejandro Gonzalez-Smith, Daniel Montoya, Grigoriy Orekhov, and Quint de Kleijn assisted with the biomechanics experiments. Michelle Ermidoro performed ADAMS analysis to quantify pedal mass effect on cycling data.

A special thanks to Karla Elias for her continued support over the last eight years. Thanks to friends and family who supported me, that understood when I had to put work on my projects, and who pushed me to finish the project strong. 


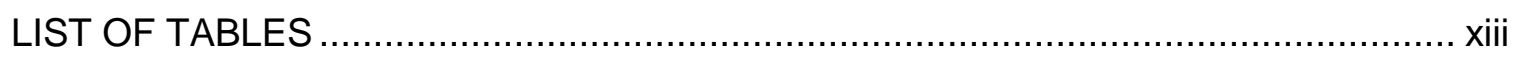

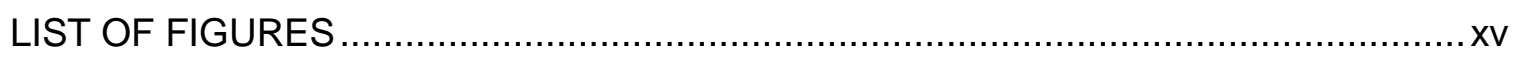

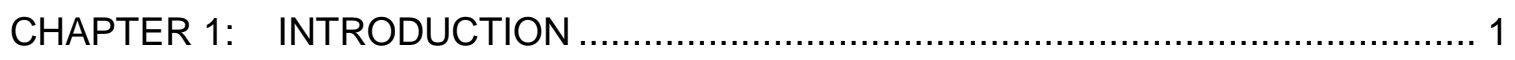

1.1 PROBLEM

1.2 PAST WORK

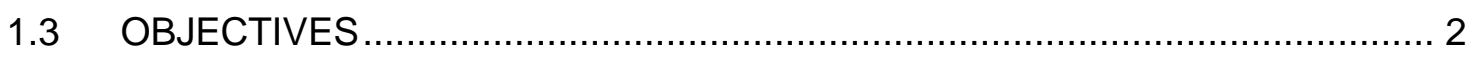

CHAPTER 2: METHODS

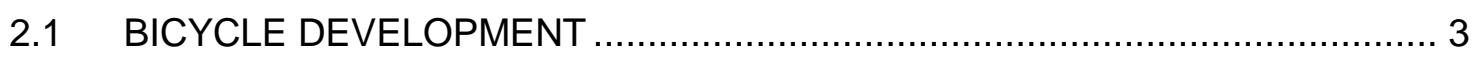

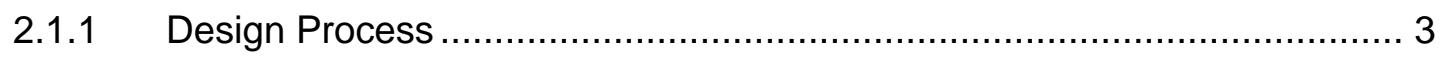

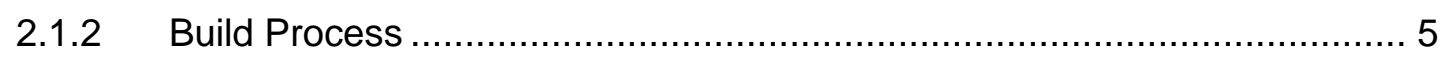

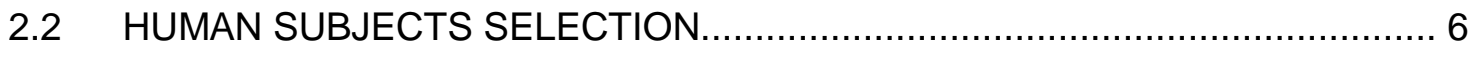

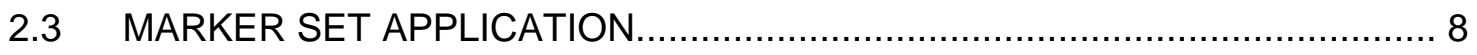

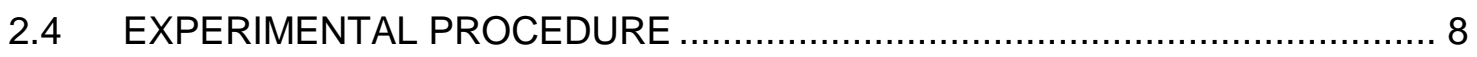

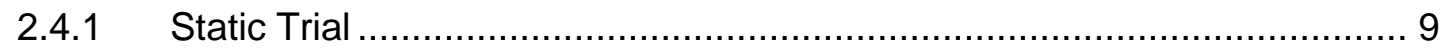

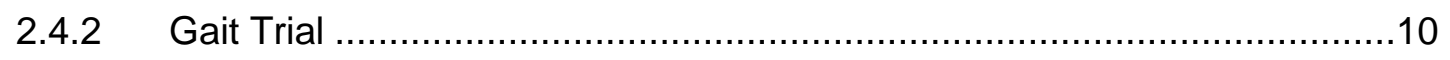

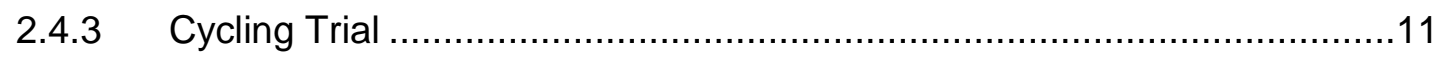

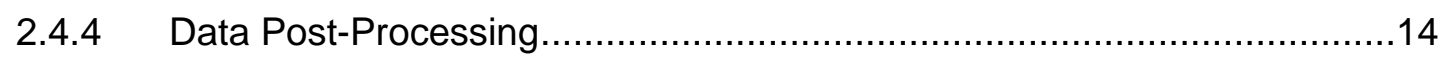

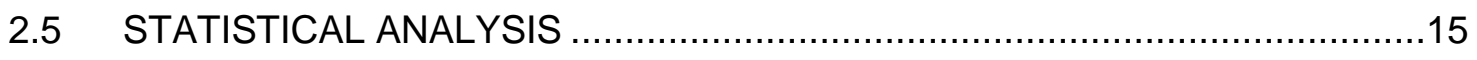

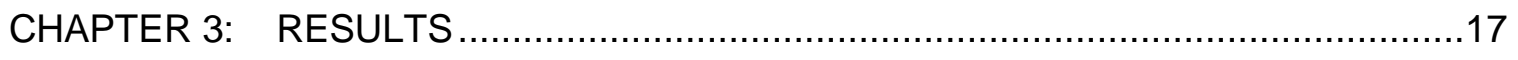

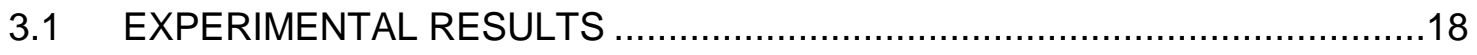




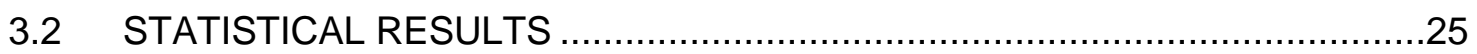

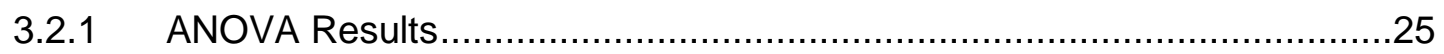

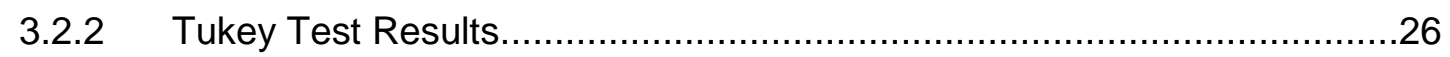

3.2.2.1 Exercise vs. BMI (Repeated Measures ANOVA + Tukey Test): ............26

3.2.2.2 NW or OB vs. Exercise (1 way ANOVA + Tukey Test):.......................26

3.2.2.3 Gait or Cycling vs. BMI (1 way ANOVA + Tukey Test): $\ldots \ldots \ldots \ldots \ldots \ldots \ldots \ldots \ldots . . .27$

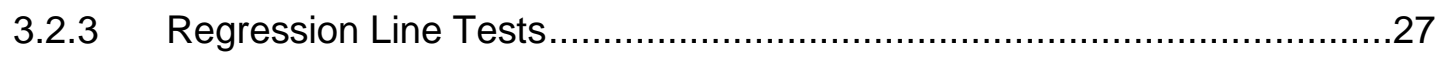

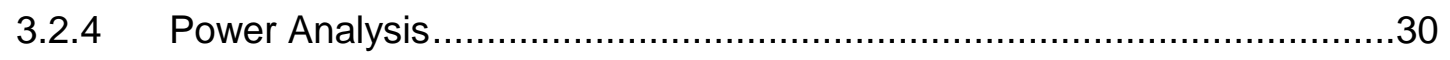

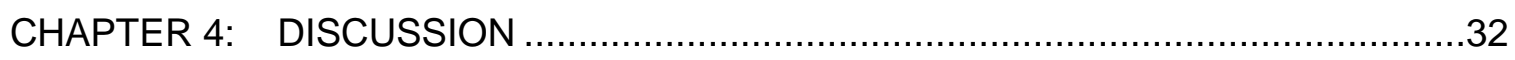

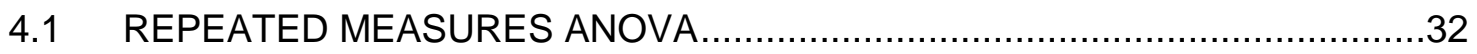

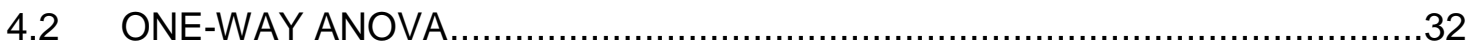

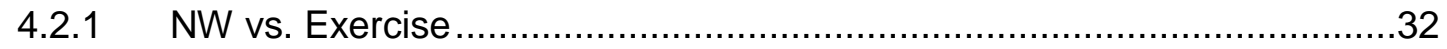

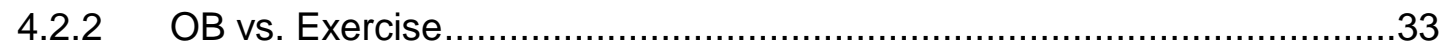

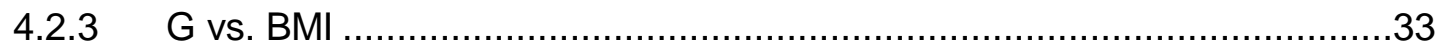

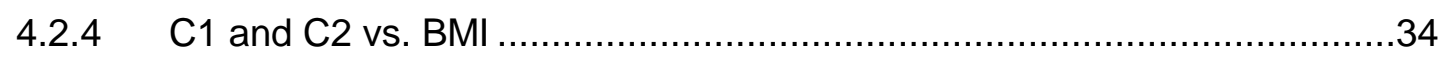

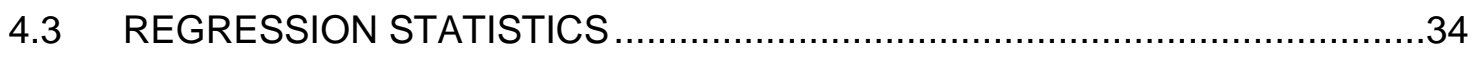

4.3.1 T-Test on the Slope of the Regression Line..........................................34

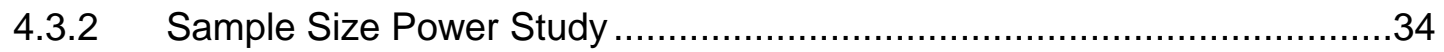

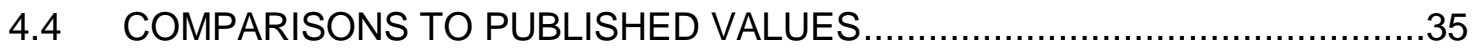

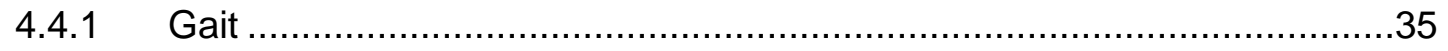

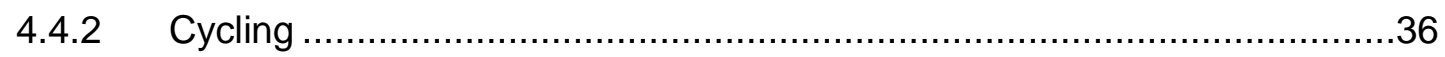




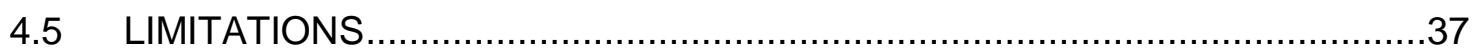

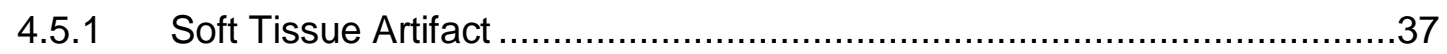

4.5.2 Marker Set Placement ....................................................................

4.5.3 Resultant Load vs. Joint Contact Force ................................................38

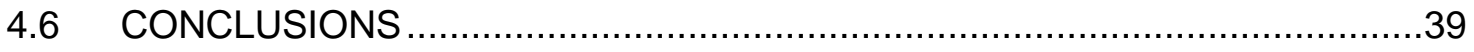

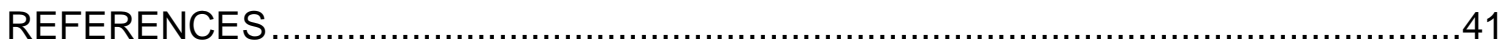

\section{APPENDICES}

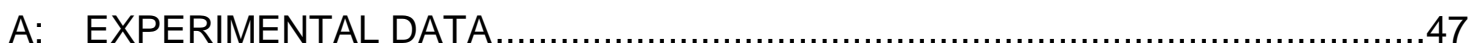

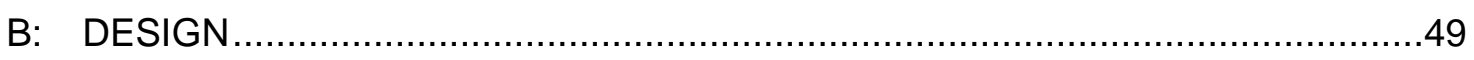

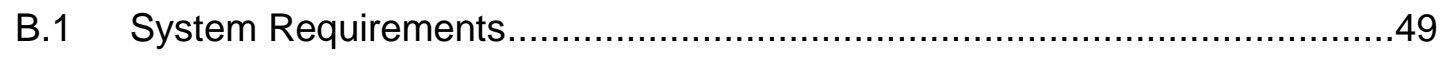

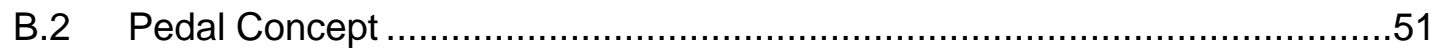

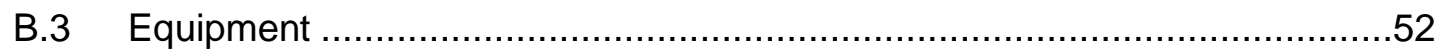

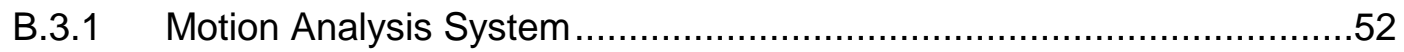

B.3.2 Load Cells

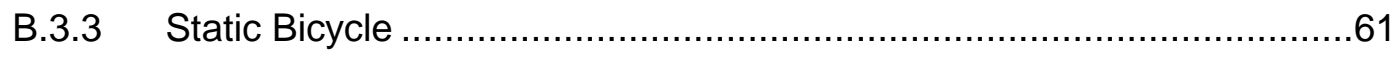

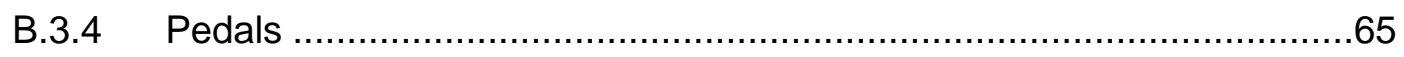

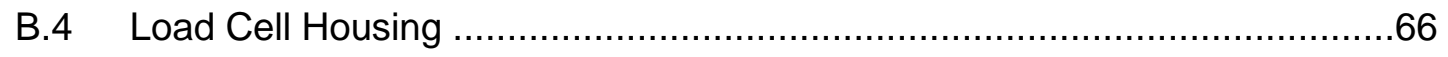

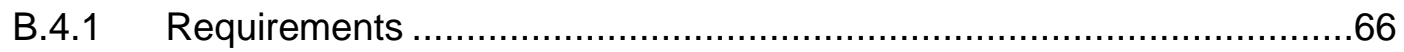

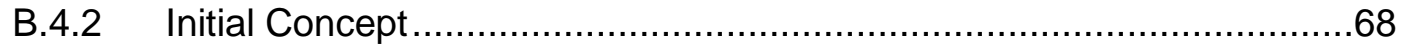

B.4.3 Load Cell Housing Geometry ……..................................................

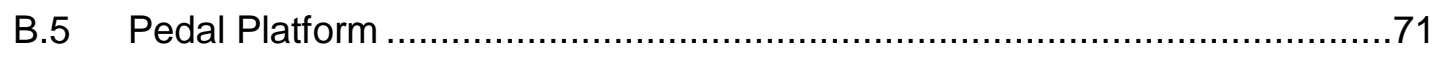




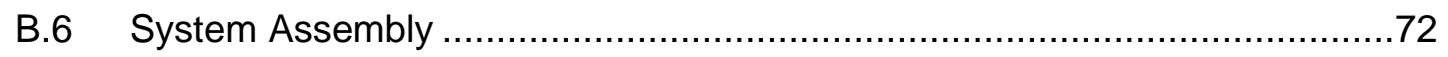

B.7 Integration with Motion Analysis System …………................................73

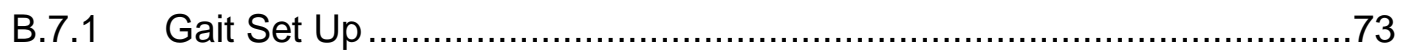

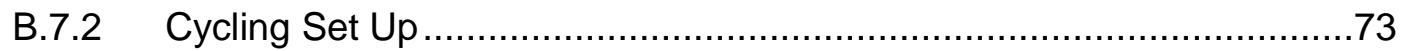

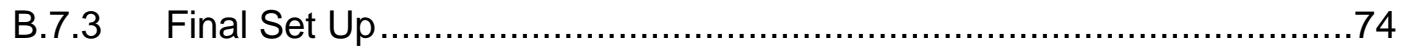

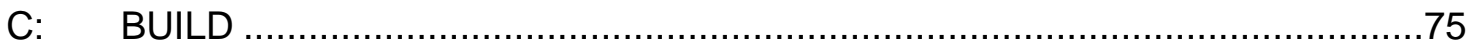

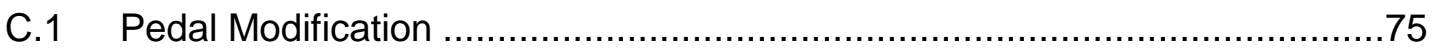

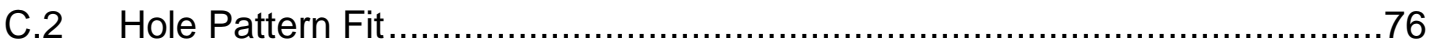

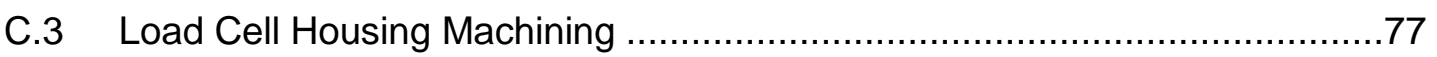

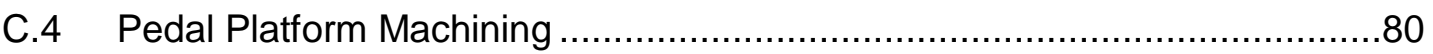

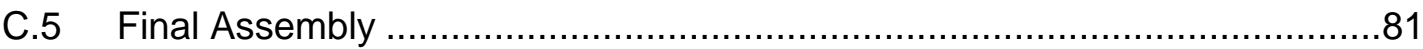

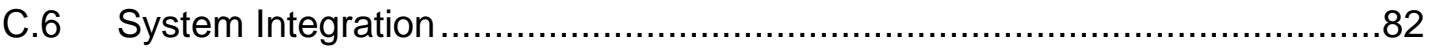

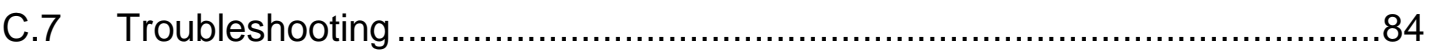

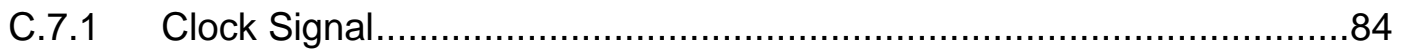

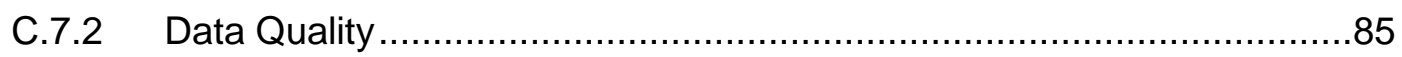

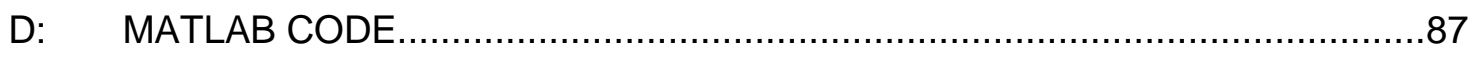

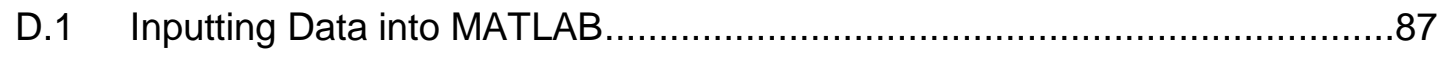

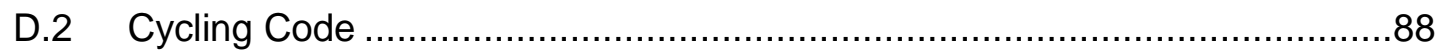

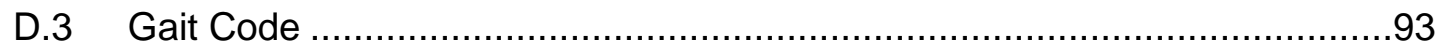

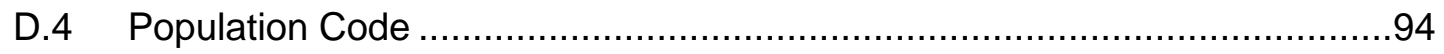

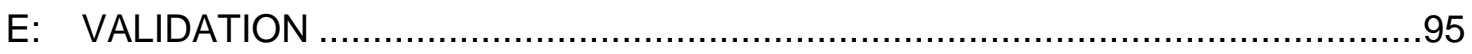




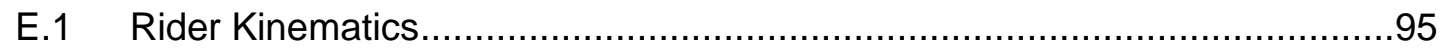

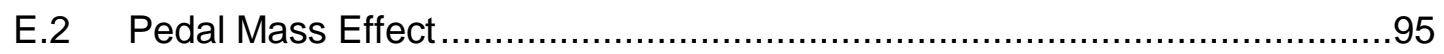

E.3 Measure Loads in Three Dimensions....................................................98

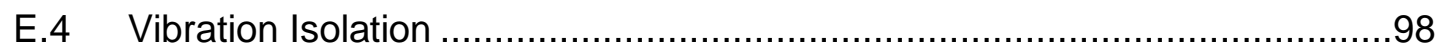

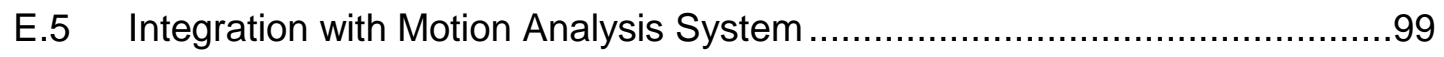

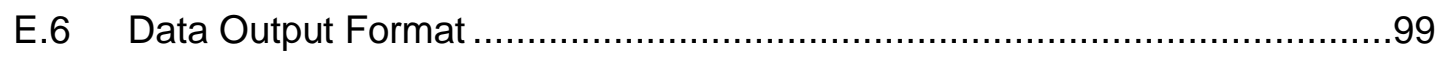

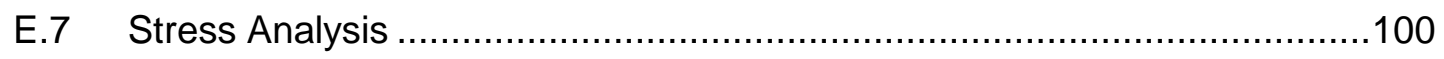

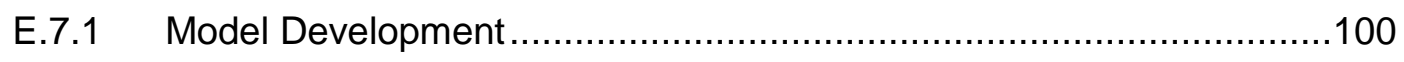

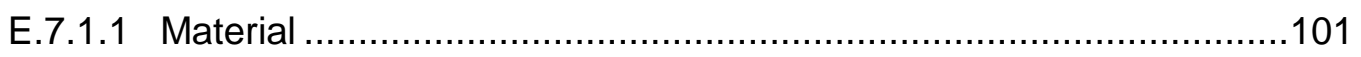

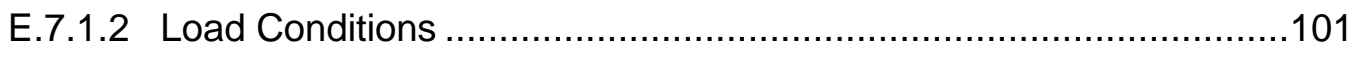

E.7.1.3 Boundary Conditions ...............................................................102

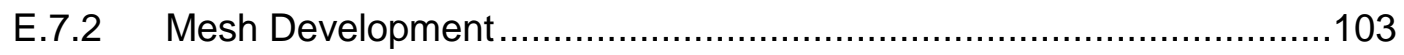

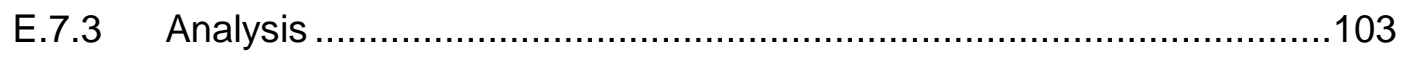

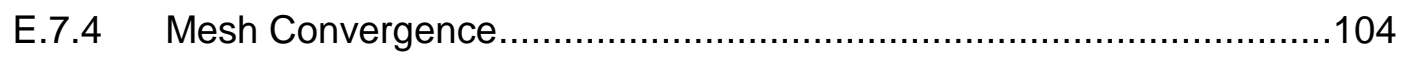

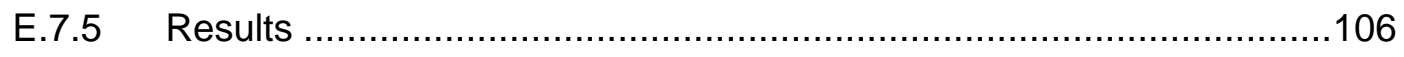

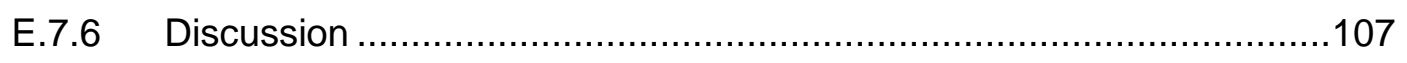

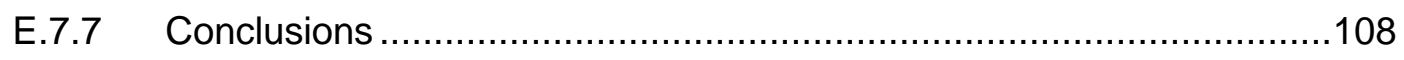

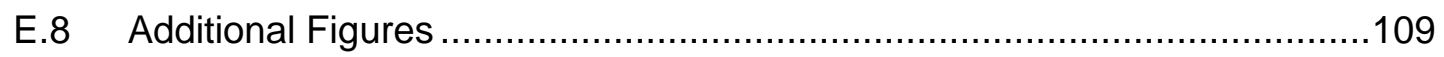

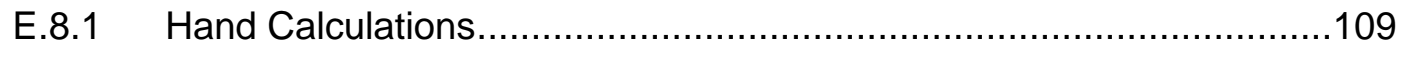

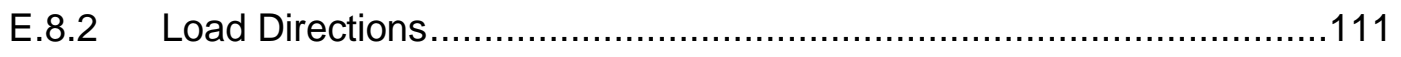

E.8.3 Degrees of Freedom vs. Seed Size ………….............................113 


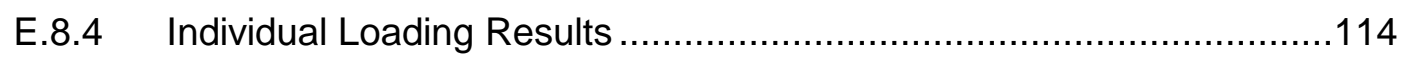




\section{LIST OF TABLES}

Table

Page

Table 2.1 Physical characteristics of subjects per population. 8

Table 3.1 Knee force, moment, and angle naming and direction

description.

Table 3.2 Reduced experimental results. Maximum values shown are

Mean \pm 1 SD

Table 3.3 ANOVA tests p-values. BMI vs Ex shows values for the repeated measures ANOVA. All other fields represent the oneway ANOVA tests. .25

Table $3.4 \quad$ T-test on the slope of the regression line for Exercise vs. BMI.

* denotes statistically significant results $(p<0.05) .+$ denotes

marginally significant results $(0.05<\mathrm{p}<0.10)$. 27

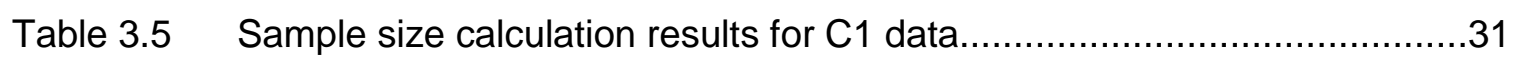

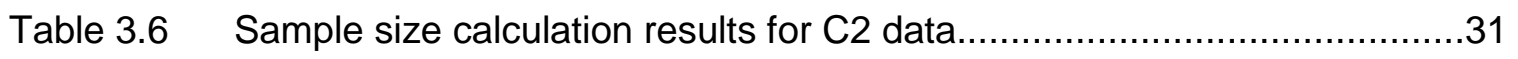

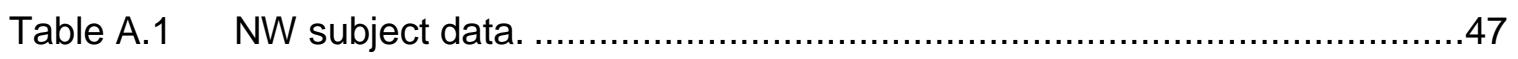

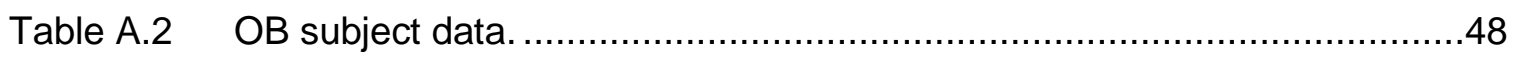

Table B.1 System requirements and testing plan..........................................49

Table B.2 Helen Hayes marker set description. .................................................56

Table B.3 AMTI AD2.5D-250 load cell maximum physical capacity........................61

Table B.4 Description of static bicycles considered.......................................64

Table C.1 Marker list for the cycling marker set based on the Helen Hayes

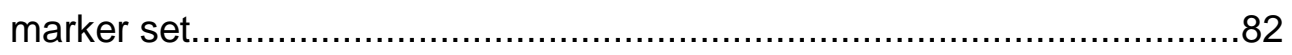

Table D.1 MATLAB cycling code expected data format...................................... 89

Table D.2 MATLAB gait code expected data format........................................94

Table E.1 Magnitude of forces created for FEA analysis.................................102 
Table E.2 Variables taken into consideration when selecting seed size. Green shows selected seed size. Difference calculated with results from previous seed size.................................................105

Table E.3 Stress, safety factor, and percent difference resulting from analysis. 


\section{LIST OF FIGURES}

$\begin{array}{lll}\text { Figure } & \text { Page }\end{array}$

Figure 2.1 Concept of load cell location within pedal body............................... 4

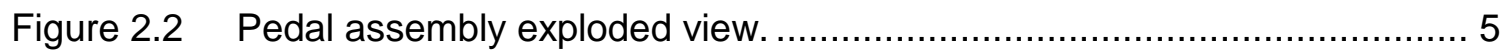

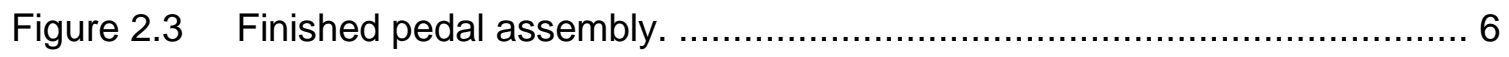

Figure 2.4 Helen Hayes marker set on subject. ........................................... 8

Figure 2.5 Cortex representation of a subject after the Sky Script is run.

Body segments are created based on the identified markers. ................. 9

Figure 2.6 Upright stationary bicycle location and set up for biomechanics experiments.

Figure 2.7 Starting position for cycling experiments. The feet of the rider

are on the frame of the bike to allow for load cell auto-zero. .13

Figure 2.8 Pedal strap is bent under itself to avoid blocking a market....................13

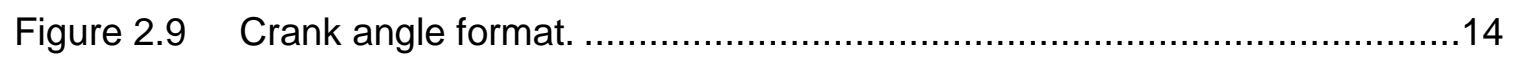

Figure 3.1 Calculated knee joint forces for normal weight and obese populations in gait and cycling. A.) Anterior force. B.) Lateral force. C.) Compressive force. Note values are mean and 1 SD.

Figure 3.2 Calculated knee joint moments for normal weight and obese populations in gait and cycling. A.) Valgus moment. B.) Extension moment. C.)External rotation moment. Note values are mean and 1 SD.

Figure 3.3 Calculated knee joint angles for normal weight and obese populations in gait and cycling. A.) Valgus angle. B.) Flexion angle. C.) Internal rotation angle. Note values are mean and 1 SD. 
Figure 3.4 Averaged gait data for NW and OB populations.

Figure 3.5 Averaged cycling data for NW and OB population at both

cycling intensities.

Figure 3.6 Knee forces for cycling and gait vs. BMI. A.) Anterior-Posterior

force. B.) Medial-Lateral force. C.) Axial (compressive) force...

Figure 3.7 Knee moments for cycling and gait vs. BMI. A.) Varus-Valgus

moment. B.) Flexion-Extension moment. C.) Internal-External

Rotation moment.

Figure B.1 Assembly with load cell on top of pedal.........................................51

Figure B.2 Proposed load cell position within pedal body.................................52

Figure B.3 Retro-reflective marker on subject. ............................................53

Figure B.4 Cortex graphical interface. Gait trial shown with markers

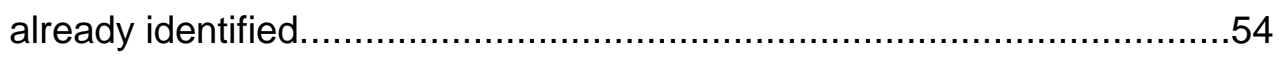

Figure B.5 Helen Hayes marker position. ................................................... 55

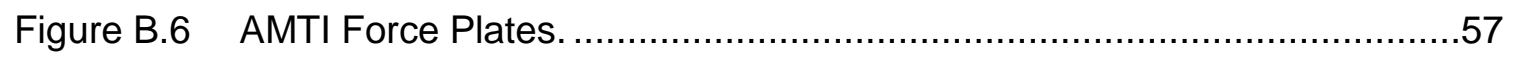

Figure B.7 Load cell and signal conditioner selected. Images from amti.biz.

Figure B.8 Life Fitness Lifecycle GX. Upright static bicycle selected. Images from lifefitness.com.

Figure B.9 Bicycles considered for project. A.) Schwinn IC2. B.) Precor UBK 615. C.) Life Fitness Lifecycle GX. D.) Life Fitness C1 Lifecycle. E.) Life Fitness C3 Lifecycle. Images obtained from website of each manufacturer.

Figure B.10 Tioga MT-ZERO pedals with Tioga ZEROaxle bearings. Image from tiogausa.com. .65

Figure B.11 Initial idea for load cell housing. 68 
Figure B.12 Final shape for load cell housing. No details are defined at this

point.......

Figure B.13 Model of pedal. Holes to accurately position in red. Bottom left

hole is used as reference point for dimensioning.

Figure B.14 Pedal hole pattern dimensions. All dimensions in inches. 69

Figure B.15 Load cell housing model. .70

Figure B.16 Pedal platform. .71

Figure B.17 Pedal assembly exploded view. .72

Figure B.18 Pedal assembly collapsed view. .72

Figure B.19 Layout of HMB Lab. .73

Figure B.20 Set up and connections needed to run a cycling biomechanics experiment. .74

Figure B.21 Equipment connections needed for experiments. Not in actual location as in the lab. .74

Figure C.1 Pedal after machining. .75

Figure C.2 A.) Wood fit test. Circled holes are used for attaching load cell housing to pedal. B.) Aluminum plate fit test. Pedal is bolted to the plate confirming the dimensions of the holes. .76

Figure C.3 Pedal housing being machined.... .77

Figure C.4 Drilling set up for pedal attachment holes. Blue tape on drill bit quickly tells depth of perforation. .78

Figure C.5 Thread tapping set up using vertical milling machine. .78

Figure C.6 Finished pedal housing. A.) and B.) show finished pedal housing. C.) shows load cell fit with housing. D.) shows housing, load cell and pedal fit. .79

Figure C.7 Pedal platform with foot strap. .80 
Figure C.8 $\quad$ A.) Housing attached to the pedal and load cell. B.) Finished right pedal assembly.

Figure C.9 Pedal marker set. .83

Figure C.10 Op-amp set up to fix triggering issue with clock signal. .85

Figure C.11 Bad quality data from initial testing. A.) Bad knee loads is repeated during several trials. B.) Load cell data missing parts.

Figure D.1 Cortex output files format. A.) DATA format. B.) TRC file format.

Figure D.2 Crank angle format. .90

Figure D.3 Cycling MATLAB code plots. A.) Data for both legs plotted against crank angle. B.) Data for three trials plotted to check for repeatability. .92

Figure E.1 ADAMS model of the crank and pedal system. .96

Figure E.2 Results of pedal mass effect in ADAMS. A.) Angular velocity reached by the model. B.) Torque requested by the controller.

Figure E.3 Pedal data recorded in three dimensions. .98

Figure E.4 Cycling data versus crank angle. A.) MATLAB output for all knee forces, moments, and angles. B.) Close up on vertical knee load. .99

Figure E.5 Load cell housing model in Abaqus. 100

Figure E.6 Partition of load cell housing and boundary conditions applied on top surface. 101

Figure E.7 Load cell housing meshed in Abaqus...........................................103

Figure E.8 Node used for mesh convergence. .104 
Figure E.9 Stress versus DOF. Note the last data point seems to converge best but there is a great increase in DOF.

Figure E.10 Nodes used to determine stresses..........................................106

Figure E.11 Combined loading results from FEA analysis...............................107 


\section{CHAPTER 1: INTRODUCTION}

\subsection{PROBLEM}

Cartilage tissue supports and distributes high loads, stabilizes and guides joint motions, and lubricates joints to provide low friction and reduce wear [1]. Osteoarthritis $(\mathrm{OA})$ is a degenerative disease of bone and cartilage and is the most common form of arthritis [2]. More than $70 \%$ of total hip and knee replacements are due to OA [2]. OA accounted for US $\$ 10.5$ billion in hospital charges in 2006 [3]. Studies have concluded that abnormal gait kinematics may cause initiation of knee $O A$ [4]. Obesity $(\mathrm{OB})$ has been identified as a risk factor for developing knee OA [2] [3] [5] [6] [7]. One identified reason why obesity increases risk of knee OA is increased knee loads accompanied by varus malalignment via increased abduction angles [8] [9].

Walking for 30 minutes or jogging for 20 minutes have been proposed as exercise regimens for weight-loss [10]. The benefits of losing weight to reduce risk of knee OA through knee load reduction have been documented. Messier et al. reported a 1:4 ratio of weight loss to load reduction, meaning for every pound of body weight reduced, the knee loads are reduced by 4 pounds [6]. However, gait is a weight-bearing exercise and OB increases knee loads that link obesity to OA [5]. Seated cycling has been proposed as a weight-loss alternative to gait since cycling produces reduced knee loads [11]. However, the lack of knowledge regarding knee biomechanics for obese subjects in weight-loss exercises other than walking serves as a barrier for identifying weight-loss exercises that may best prevent knee OA. 


\subsection{PAST WORK}

There exists a lack of studies that have directly compared knee loads for obese subjects in gait and cycling. Some studies have looked at the effect of obesity on knee loads during gait. For example, Browning and Kram observed the effects of obesity in gait at different walking speeds [5]. Other studies have explored knee loads during cycling. Davis and Hull modified a bicycle to measure foot loads [12] and Ruby et al. developed a three dimensional model for estimating knee loads during cycling [13]. Studies that used rider weight as a factor focused on the effect of rider mass on the frame of the bicycle [14], however, no studies were found that explored the effects of obesity on knee loads during cycling.

\subsection{OBJECTIVES}

The long term goal of this work is to determine if cycling is a better weight-loss exercise to walking for $\mathrm{OB}$ subjects in the context of reducing $\mathrm{OA}$ incidence via a reduction in knee loads. As a crucial first step towards achieving that long-term goal, the objectives of this study are to (1) modify an upright stationary bicycle to measure forces and moments at the pedals in three dimensions, (2) conduct pilot experiments with a motion capture system to calculate internal knee joint loads during cycling, and (3) compare knee loads for normal weight and obese populations during gait and cycling. The results will be used to design a more comprehensive study of kinetic and kinematic differences in gait, cycling, and elliptical training for normal weight and obese subjects. 


\section{CHAPTER 2: METHODS}

As discussed in chapter 1 , obese subjects have higher knee loads while walking compared to normal weight individuals [5]. Higher knee loads are linked with increased risk for knee OA [5] [6]. Since cycling has been shown to produce lower knee loads than gait [11], cycling may be a preferred weight-loss exercise. Knee loads are defined as resultant knee joint forces and moments produced by ground reaction forces (GRF) and inertial effects and, thus, do not include the effects of muscle forces which normally result in higher joint contact loads. Thus, the hypotheses of this study are (1) cycling produces lower knee resultant loads than gait for both normal weight and obese subjects, (2) obese subjects will have higher knee loads than normal weight subjects when cycling, and (3) obese subjects will have higher knee loads than normal weight subjects when walking. To address the hypotheses, the objectives of this study are to 1) design, build, and implement 3-dimensional load measuring pedals (measure forces and moments) and 2) compare knee resultant loads and knee angles during gait and cycling for normal weight and obese subjects. Protocols pre-approved by the Cal Poly Human Subject Committee were followed to minimize risk to human subjects.

\subsection{BICYCLE DEVELOPMENT}

An upright stationary bicycle was developed to perform cycling biomechanics experiments. The bicycle is integrated with a full Motion Analysis system and measures three force components and three moment components at the pedals. The following section briefly describes the design and build process. A detailed description of the design and build process is provided in Appendix B and Appendix C, respectively.

\subsubsection{Design Process}

A set of requirements were developed to improve the quality of the data measured in cycling experiments. The bicycle must not change rider kinematics. The bike pedal must 
measure three force components and three moment components and the data must be filtered to reduce noise artifacts. The bike must be integrated with the motion analysis system present at the HMB lab. The data should be outputted against crank angle (a standard format). The modified pedal must support the expected loads.

The static bicycle chosen is the Life Fitness LifeCycle GX (Life Fitness, Rosemont, IL). This bike was chosen mainly for its ease to retrofit and repeatability in resistance level selection. Two AMTI AD2.5D-250 load cells (AMTI, Watertown, MA) were selected to measure the forces and moments at the pedals. These load cells measure forces and moments in three-dimensions and use GEN 5 signal conditioners to filter data. To keep rider kinematics similar to use with the stock pedals, it was decided to use spindle-less pedals and locate the load cells through the pedal body so the top pedal assembly is at

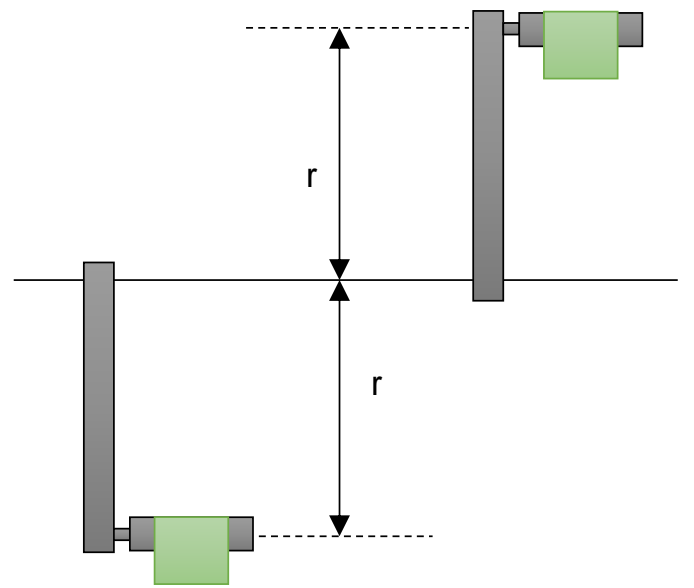

Figure 2.1 Concept of load cell location within the same location as the original pedal pedalbody.

surface (see figure 2.1). Thus the distance between the feet and the crank center is the same as with the stock pedal at Top Dead Center (TDC) and at Bottom Dead Center (BDC). Tioga MT-ZERO pedals were selected because they have no spindle and have oversized bearings that can support the expected loads. Load cell housings were created to attach the load cell to the pedal. The load cell housing geometry was developed to place the foot of the rider at the original location of the pedal surface.

The HMB lab has a full Motion Analysis system (Motion Analysis Corporation, Santa Rosa, CA). The motion analysis system uses the motion analysis software Cortex. The AMTI load cells are integrated with Cortex via a USB connection. Cortex is able to 
track the location and orientation of the load cells. The load cell integration with Cortex and the motion analysis system is described in detail in Appendix B.7. A custom MATLAB code was created to output data against crank angle. This code also organizes, interpolates, and averages data for all subjects. The MATLAB code is described in Appendix D. Stress analysis was performed to demonstrate that the load cell housing created can withstand the expected loads. This analysis and other validation tests for the requirements mentioned above are discussed in Appendix $\mathrm{E}$.

\subsubsection{Build Process}

The exploded view of the pedal assembly is shown in figure 2.2. The modified pedal assembly is designed to easily be put on any standard bicycle crank. The load cell is attached to the load cell housing. The housing is attached to the pedals. A pedal platform was developed to allow for strapped cycling. The pedal platform is attached at the top of the load cell. Only the pedal platform is in contact with the top of the load cell to allow for reliable and repeatable measurements.

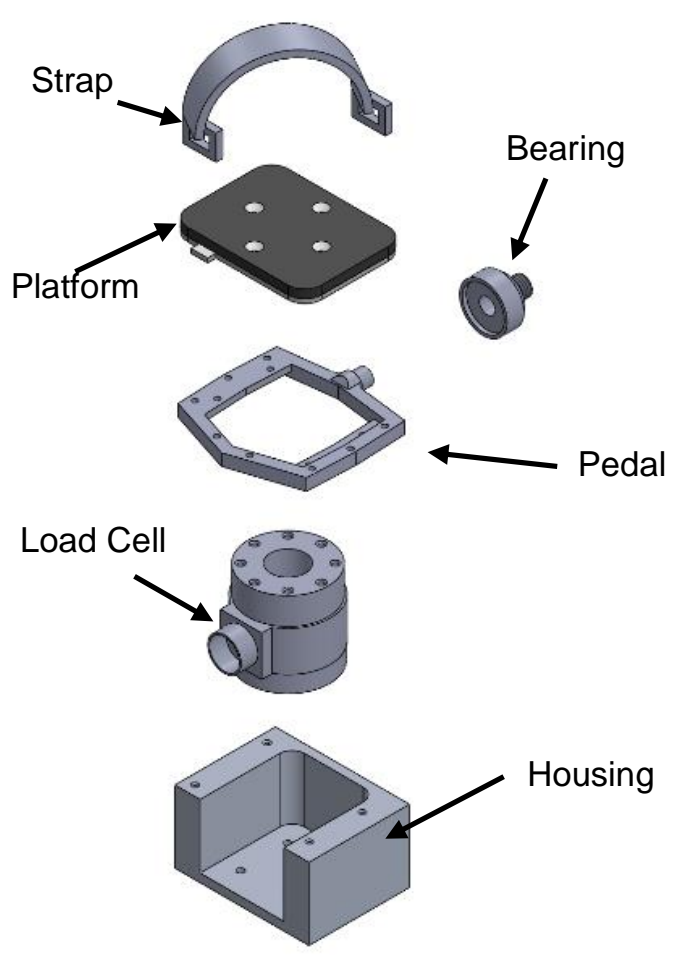

Figure 2.2 Pedal assembly exploded view. 
Figure 2.3 shows the pedal assembly. The load cell housing was fabricated from a block of aluminim 6061 . The machining was done in a vertical milling machine. The TIOGA pedals had the center of the pedal body removed with a pneumatic cutoff wheel and was deburred using a Dremel. The pedal platforms were made from an aluminum plate with a layer of nitrile rubber. The aluminum provided rigidity whie the rubber provided comfort and grip for the rider.

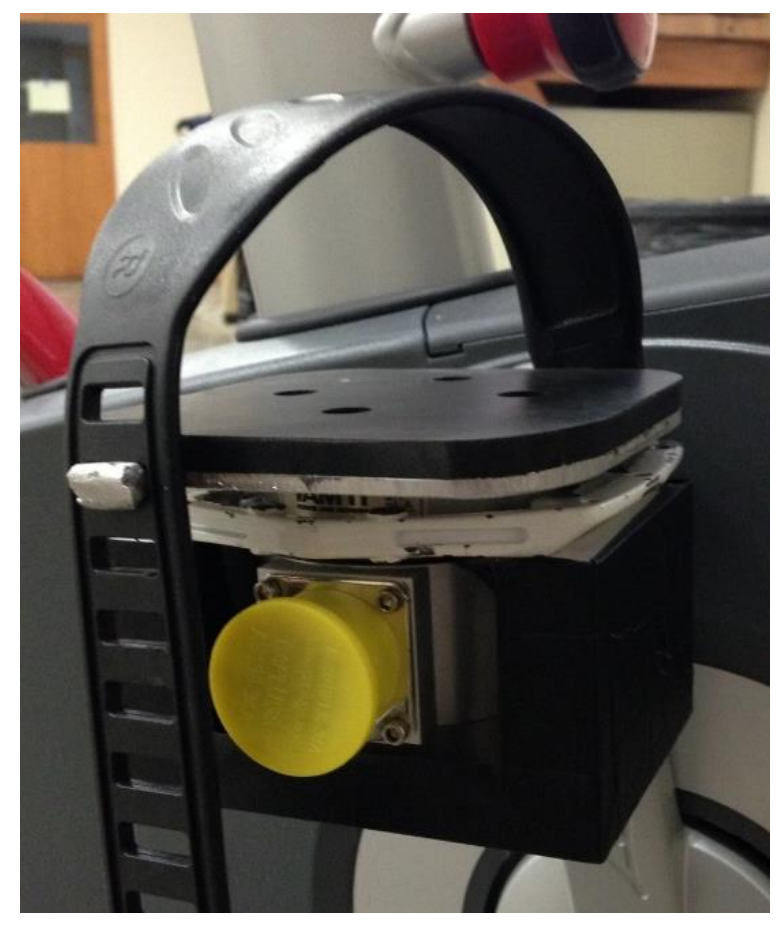

Figure 2.3 Finished pedal assembly.

Cycling expeirments were performed once the pedal assembly was attached on the stationary bicycle and the load cells were recognized by Cortex. The following sections describe the gait and cycling biomechanics experiments performed.

\subsection{HUMAN SUBJECTS SELECTION}

Two subject populations were recruited: normal weight $(N W ; n=4)$ and obese $(\mathrm{OB} ; \mathrm{n}=4)$. Body Mass Index $(\mathrm{BMI})$ was used to determine a subject's membership to a population (see table 2.1). BMI is calculated using equation 2.1

$$
B M I=\frac{\text { weight }(\mathrm{kg})}{\operatorname{height}^{2}\left(\mathrm{~m}^{2}\right)}
$$

Volunteers were given an overview of the study and eligibility was determined by asking questions pertaining to medical history and ability to engage in physical activity. Eligible volunteers received a detailed description of the study and consent forms were reviewed. Informed consent and photographic release were obtained. All subjects had to 
answer and pass all questions in the Physical Activity Readiness Questionnaire (PAR-Q) in order to participate in this study. Finally, the subjects completed preliminary tests including body weight, height, and knee joint Q-angle measurements (angle between lines created by the ASIS, the midpoint of the patella, and the tibial tuberosity). All subjects that participated in this study were male in order to avoid gender-related biomechanical differences that are known to exist in gait [15]. Inclusion and exclusion criteria are described below:

$\underline{\text { Inclusion Criteria }}$

- $\mathrm{NW}: \mathrm{BMI}=18.5-25.0 \mathrm{~kg} / \mathrm{m}^{2} ; \mathrm{OB}: \mathrm{BMI}=30.0-40.0 \mathrm{~kg} / \mathrm{m}^{2}$

- Age: 18 - 40 years

- English speaking

\section{Exclusion Criteria}

- Cardiovascular or respiratory disease

- Metabolic disease or complication

- Weight loss or weight gain in previous 6 months

- History of major psychiatric illness, drug abuse, or unsafe dieting practices

- Major medical conditions that prohibit physical activity

- Pregnant women or women expecting or trying to become pregnant

- Pre-existing conditions producing abnormal knee loading (e.g. varus-valgus misalignment)

- Other joint injuries, etc. (other than overweight/obese) 
Table 2.1 Physical characteristics of subjects per population.

\begin{tabular}{|l|r|r|}
\hline \multirow{2}{*}{ Item } & \multicolumn{2}{c|}{ Mean \pm SD } \\
\cline { 2 - 3 } & \multicolumn{1}{c|}{ NW } & \multicolumn{1}{c|}{ OB } \\
\hline Age (years) & $22.3 \pm 1.9$ & $23.3 \pm 2.1$ \\
\hline Height $(\mathrm{m})$ & $1.85 \pm 0.06$ & $1.81 \pm 0.03$ \\
\hline Mass $(\mathrm{kg})$ & $79.9 \pm 5.8$ & $120.7 \pm 5.8$ \\
\hline BMI $\left(\mathrm{kg} / \mathbf{m}^{2}\right)$ & $23.5 \pm 2.8$ & $36.7 \pm 1.8$ \\
\hline
\end{tabular}

\subsection{MARKER SET APPLICATION}

To be able to use the Sky Scripts in Cortex that model the human body, the lower body Helen Hayes $(\mathrm{HH})$ marker set was selected. This marker set models the legs, which is all that is needed to find knee joint loads. The lower body $\mathrm{HH}$ marker set is described in detail in Appendix B.3.1. Subjects wore compression clothing and the markers were placed at standard anatomical landmarks. Figure 2.4 shows a subject wearing compression clothing and with the lower body Helen Hayes marker set.

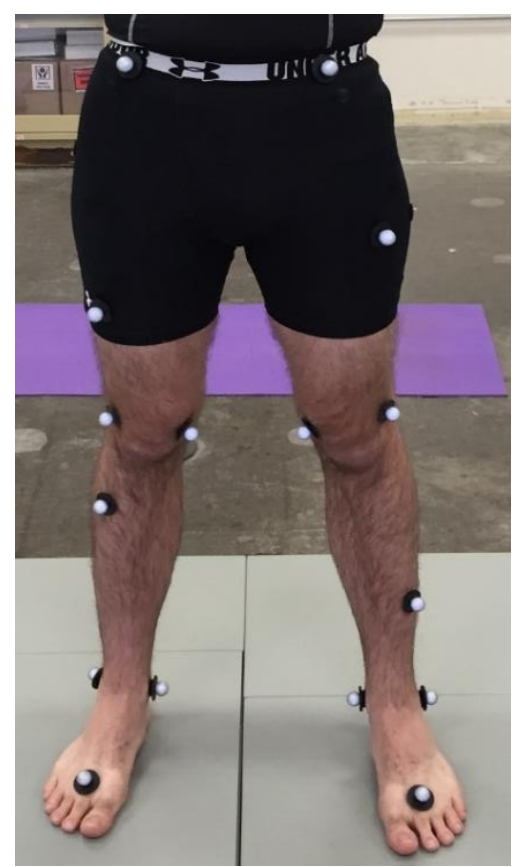

Figure 2.4 Helen Hayes marker set on subject.

\subsection{EXPERIMENTAL PROCEDURE}

The experimental procedure consisted of three parts: Static Trial, Gait, and Cycling experiments. A description of each part is given below. Every subject, regardless of the population, must have their data be untraceable to their personal information (name, address, age, etc.). To this end, subjects were identified with a naming convention. This naming format starts with the four digit year of the data capture, followed by the first three letters of the month, and ending with the two-digit date of the day the trial took place. To account for multiple subjects coming into the lab on the same day, a number is added at the end to identify which captures correspond to which subject. For example, 2015Nov23- 
2 is the second subject whose data was recorded on November 23, 2015. Three sets of captures are taken for all subjects; to identify them, the words "Static", "Gait\#", and "Cycling\#" are added after the date and separated with an underscore. The numbers after Gait and Cycling are explained in their appropriate section.

A full motion analysis system (Motion Analysis Corporation, Santa Rosa, CA) was used for these experiments. The motion analysis system includes the motion analysis software Cortex, eight near-infrared cameras, two AMTI Accugait force plates (AMTI, Watertown, MA), and a modified upright stationary bicycle (Life Fitness, Rosemont, IL). This system is described in detail in the appendix B.3.1. The full design, build, and validation of the modified static bicycle is found in Appendix B through Appendix $E$.

\subsubsection{Static Trial}

The static trial refers to a short motion capture (about 3 seconds). The subject must have the retro-reflective markers on in the lower body $\mathrm{HH}$ marker set pattern to record the static trial. The subject was asked to stand in the center of the lab over the Accugait force plates (in the capture volume). The capture is taken as the subject stands still with the arms raised to avoid the hands from covering any markers. The markers were identified and their trajectories were processed (rectified, cubic joined, and smoothed - see Appendix B.3.1 for more information). The “HH_StaticToDynamic.Sky" Sky Script under the KinTools RT library in Cortex was run to create the knee and ankle joint centers and to define the axis of rotation of these joints. Figure 2.5 shows the visual result of running this script in Cortex.

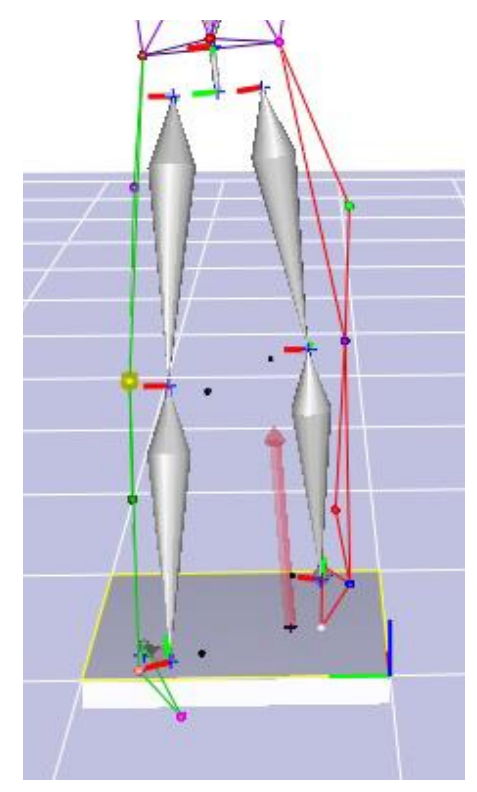

Figure $2.5 \quad$ Cortex representation of a subject after the Sky Script is run. Body segments are created based on the identified markers. 


\subsubsection{Gait Trial}

Once the static trial was taken, the medial markers (knee and ankle) were removed. Since the knee and ankle joints travel near the corresponding joint on the opposite leg, the subjects may expand their stance to avoid hitting the medial markers together. This stance expansion is not desired as it is a change from normal gait biomechanics. Removing the four medial markers removes this artifact. The "HH_StaticToDynamic.Sky" was used because the medial markers were removed. Cortex uses the lateral and medial knee and ankle markers to define the axis of rotation for each joint. If the medial markers are removed, Cortex uses this Sky Script to define the axis of rotation of the joint with respect to the remaining markers.

While the static trial was being processed in Cortex, the subjects were asked to walk across the force plates to figure out a starting position such that their gait was not altered due to targeting the force plates. Gait trial recordings were started when the subject felt comfortable with the starting distance and had completed a few successful gait trials. For each subject, three successful trials were recorded for averaging. The trial number was listed at the end of the file name. For example, 2015Nov23-2_Gait3 contains the data for the third gait trial for the second subject recorded on November 23, 2015.

Once three trials were recorded, the captures were trimmed to only contain frames where the subject is in the capture volume. The markers were identified and postprocessed in Cortex (using the "rectify trajectories", "cubic join missing frames", and "smooth trajectories" options). The inverse dynamics solver was activated to calculate knee loads. Data was exported in TRC files (marker positions - kinematic data) and in DATA files (knee loads and Ground Reaction Force data - kinetic data). These file extensions are explained in B.3.1. The frames with force plate data corresponding to the first and last heel strike in the trial were noted. These frames were inputted into the 
MATLAB code to create a percent stride vector and allow proper data formatting for plotting. The MATLAB code is explained in detail in Appendix D.

\subsubsection{Cycling Trial}

After three gait trials were captured, the modified upright static bicycle was moved into the capture volume. The bicycle was placed pointing towards the HMB computer as seen in the schematic in figure B.20 (Appendix B.7). Care was taken so that the bicycle ground supports were not resting on a force plate. The wires that connect the load cells to the GEN 5 signal conditioners were extended outward, perpendicular to the orientation of the bike in the room to minimize effects of cable inertia or tension on the pedals. The ground supports of the bike were adjusted so the bike does not rock back and forth during use. This set up is shown in figure 2.6. A step stool was provided to facilitate getting on and off the bicycle. The pedals should not be used as a step to get on or off the bike as overloading may occur risking the integrity of the load cells placed at the pedals. The stationary bicycle was sized for each subject by adjusting the seat and handle bar positions so that when the pedal is at bottom dead center (BDC) the knee is almost fully extended, but not locked into extension. The ball of the foot should rest above of the axis of rotation of the pedal.

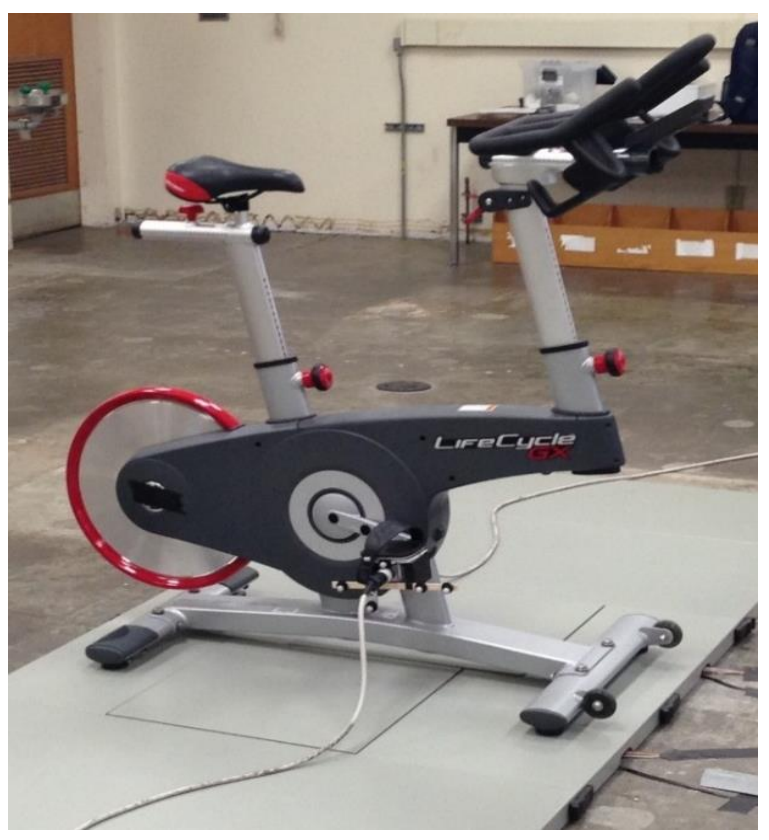

Figure 2.6 Upright stationary bicycle location and set up for biomechanics experiments.

Two cycling captures were recorded for each subject. Each capture contains three crank cycles (trials) used for averaging and processing. Subjects were asked to reach 70 RPM and hold that cadence as steady as possible on both captures. This cadence was 
chosen because it is considered a moderate cadence. The first capture, named "Cycling1", corresponds to cycling at a resistance level of 10 out of 20 . This resistance level is considered low intensity. The high inertia of the flywheel at the back of the bike makes it so that little effort is needed to maintain a steady cadence. This high inertia was also a cause of concern as the pedals may remain in motion if the feet are removed suddenly. If this happens the pedals may strike the feet or legs of the rider causing injuries. The riders were told to slow down the bike in a controlled manner when the trial is finished to avoid injury. The emergency brake was recommened as the best way to slow the bicycle down. The second trial, named "Cycling2", corresponds to a cycling resistance of 15 out 20. This resistance level is considered moderate as pilot experiments showed could be held by an average person for about 30 minutes.

The subjects started with their feet up on the frame of the bike so that nothing was resting or touching the pedals (see figure 2.7). The ten pedal markers had to be seen at the first frame of the trial. The first frame was used to auto-zero the load cell data. If the ten markers were not seen, Cortex cannot create a virtual model of the load cells and therefore cannot locate the force vector from the load cells in the virtual representation of the lab space. Without this vector, auto-zeroing cannot be done. The load cells were also auto-zeroed by pressing auto-zero botton on the GEN 5 signal conditioners. With autozeroing done and the markers visible, the capture was started. At this point, the subject was able to put their feet on the pedals and HMB lab staff then strapped the subject to the pedals. The long end of the pedal strap was flipped into itself to keep it from covering a marker (see figure 2.8). As the HMB staff walked away from the bike, the load cells wires were extended outwards, and the motion capture was started. 


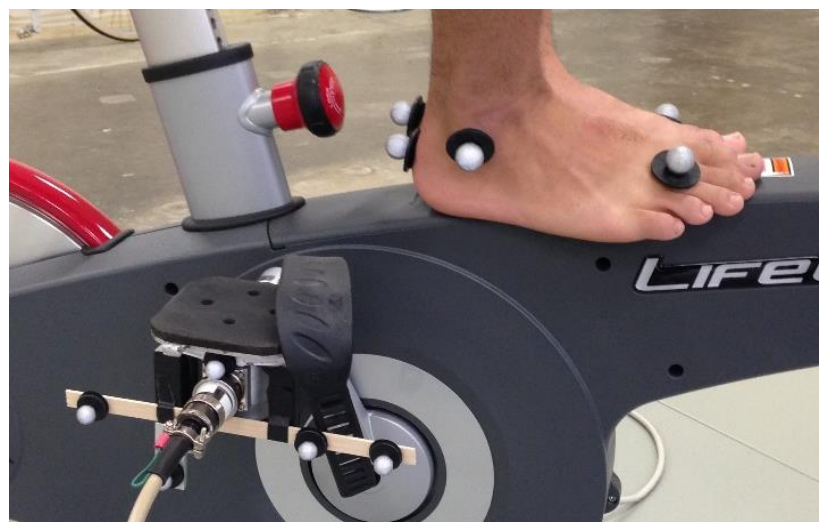

Figure 2.7 Starting position for cycling experiments. The feet of the rider are on the frame of the bike to allow for load cell auto-zero.

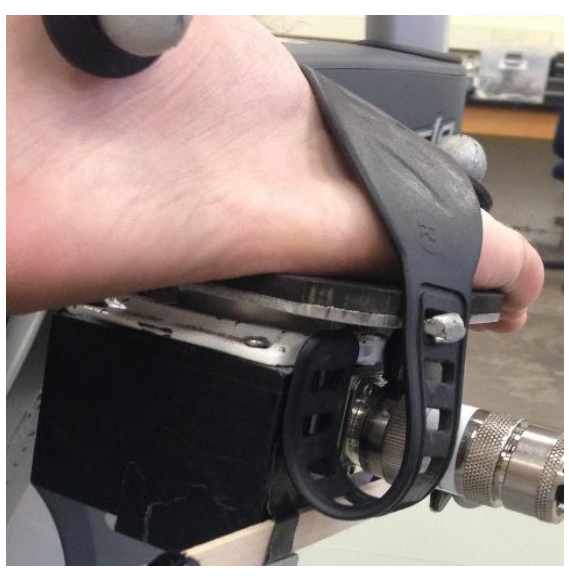

Figure 2.8 Pedal strap is bent under itself to avoid blocking a market.

The subject was asked to start pedaling to reach 70 RPM and to maintain that cadence with a resistance level of 10 out of 20 . Subjects obtained feedback on their cadence from the computer display of the bicycle. The subjects were asked to anounce when 70 RPM was being held constant, i.e. that the subject was in steady state cycling. The recording time of this event was noted. After this point, and extra 5 to 15 seconds were allowed to pass to ensure steady state was reached. From this point on, 15 more seconds of steady state cycling were recorded and the capture was ended. Lastly, the subject was unstrapped from the pedals. Only the data during the last 15 seconds of steady state cycling was used for processing. No trimming of the capture was done. To select this data, the frames corresponding to this time location were noted and inputted into the MATLAB code. The process of the last two paragraphs was repeated once more with a resistance level of 15 out of 20 (to record the second capture - Cycling2).

Marker trajectories were processed similarly to the gait data. The only difference was that cycling data had ten extra markers for the pedals, which changes the marker set used in Cortex. Once again, TRC and DATA files were exported to use in MATLAB. In cycling, a crank cycle (full revolution) is a trial, therefore many trials were recorded in a single capture. In gait, each capture contains a single trial. 


\subsubsection{Data Post-Processing}

The kinetic (force plate and load cell data) and kinematic (camera data or marker trajectories) data were exported as DATA and TRC files, respectively. These files were brought into a custom MATLAB code for post-processing of the data. The code formatted the TRC and DATA files for use in MATLAB, then selected the data from the range of frames given in the user input. The crank angle and percent stride vectors were created based on TRC data. The crank angle format is shown in figure 2.9. Top dead center (TDC) is defined as zero degrees. Percent stride starts at heel strike. The data was organized in matrices with the format expected by the rest of the code. Interpolation was used to have all the data shown at the same crank angles for cycling and percent stride for gait. The code averaged data for three gait trials and three cycling trials with both resistance levels and plotted the averaged data. Knee results (three force components, three moment components, and three angles) were plotted against crank angle for cycling data and percent stride for gait data. Another MATLAB code averaged the data for populations (NW and $\mathrm{OB}$ ) and exported maximum and minimum values for all knee forces, moments, and angles for each subject in a Comma Separated Values (CSV) file that was later used in the statistical analysis. A full discussion and detailed description of the MATLAB code is given in Appendix D.

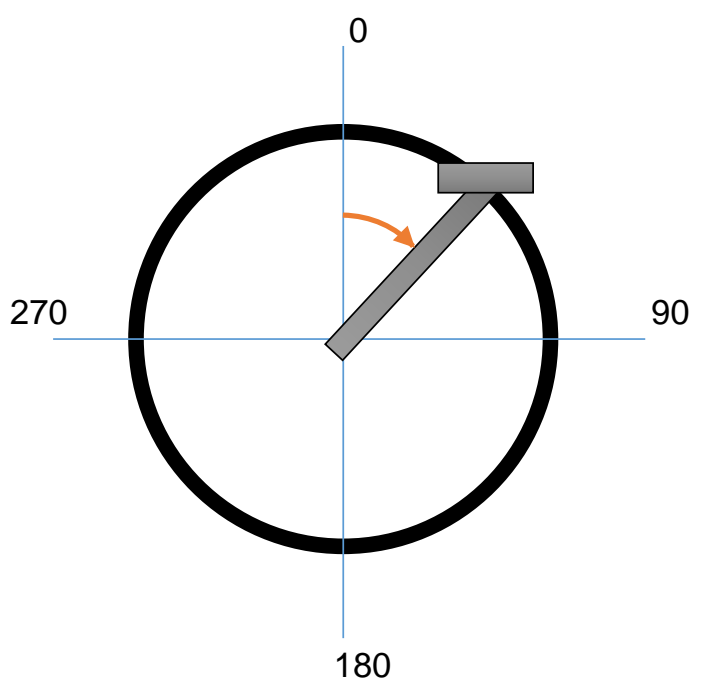

Figure 2.9 Crank angle format. 


\subsection{STATISTICAL ANALYSIS}

The maximum and minimum values of all sets of data were opened in Excel. The magnitude of the maximum and minimum values for each load of each subject were compared. The load with the largest magnitude was selected. The largest axial load was always compression, whereas for Anterior-Posterior and Medial-Lateral shear loads the magnitude and not direction was considered most important to study in the context of cartilage damage. The values with largest magnitude in each force, moment, and angle for each subject were organized in Excel for subsequent statistical analysis. There were two factors in the analysis: BMI (NW and $\mathrm{OB})$ and Exercise. The exercise factor had three levels: Gait, Cycling1 (resistance level of 10 - low intensity cycling) and Cycling2 (resistance level of 15 - moderate intensity cycling). These exercises are hereafter referred to as $\mathrm{G}, \mathrm{C} 1$ and $\mathrm{C} 2$.

Minitab 17 (Minitab Inc., State College, PA) was used for statistical analysis of experimental data. A repeated measures ANOVA (Analysis of Variance) was done to explore the interactions between BMI and Exercise. The repeated factor was the exercise level because each subject performed all the exercises. The data was also organized in different ways to run one-way ANOVA tests. Three one-way ANOVAs were performed for $\mathrm{C} 1, \mathrm{C} 2$, and $\mathrm{G}$ versus $\mathrm{BMI}$ to look for differences between $\mathrm{OB}$ and NW in each exercise. Two one-way ANOVAs were performed for OB and NW versus Exercise to look for differences between $\mathrm{G}, \mathrm{C} 1$, and $\mathrm{C} 2$ in NW and $\mathrm{OB}$ populations. These latter one-way ANOVAs were conducted so that if a difference is observed in the NW data, it would be checked against the OB data to see if the result is observed in both populations. For example, if the vertical knee force in the $\mathrm{OB}$ data is larger for $\mathrm{G}$ than it is for $\mathrm{C} 1$ or $\mathrm{C} 2$, then the same load is compared in the NW data to see if this trend or result is observed as well. Statistical significance was defined as $p<0.05$. Any significant result seen at any 
step in the ANOVA tests was followed up with a Post-hoc Tukey test. Next, the maximum values of all loads for all subjects were plotted against BMI. A t-test was done on the slope of the regression line. The t-test was performed in Excel, using the "Regression" option under the "Data Analysis" menu. Again, statistical significance was define as $p<0.05$. The t-test on the slope of the regression line was done to look for correlations between maximum loads and BMI. The slope of the regression line shows if there is a trend in the data. If the slope of the regression line is equal to zero, no trend is present in the data. This t-test shows if the slope of the regression line is statistically different to zero.

As a final test, a power study was performed to obtain sample sizes needed to observe significant differences in each knee load (force and moments) for both populations. The "Two sample using average values" calculator by DSS Research [16] was used for this analysis. This calculator requires the average and standard deviation (SD) for the two groups being compared. In this case, the groups were NW and OB. The alpha or confidence level, $\alpha$, was set to $5 \%$ which corresponds to a $95 \%$ confidence interval. The beta error level was set to $20 \%$ which corresponds to $80 \%$ statistical power. 


\section{CHAPTER 3: RESULTS}

The gait and cycling biomechanics experiments investigated the effects of BMI and Exercise (Ex) type on knee joint loads and angles. The experimental results were summarized using mean and one standard deviation. Reduced results are reported below. Comprehensive experimental data is listed in Appendix A.

The positive directions for Anterior-Posterior $\left(F_{A-P}\right)$ and Medial-Lateral $\left(F_{M-L}\right)$ force components were the anterior and lateral directions, respectively. The axial $\left(F_{A x}\right)$ component was defined as positive for compression. Valgus, extension, and external rotation were positive directions for Varus-Valgus ( $\left.\mathrm{M}_{\mathrm{V}-\mathrm{V}}\right)$, Flexion-Extension ( $\left.\mathrm{M}_{\mathrm{F}-\mathrm{E}}\right)$, and Internal-External Rotation ( $\left.\mathrm{M}_{\mathrm{IR}-\mathrm{ER}}\right)$ moments, respectively. Similarly, valgus, flexion, and internal rotation were defined as positive directions for Varus-Valgus (V-V angle), FlexionExtension (F-E angle), and Internal-External Rotation (IR-ER angle) angles, respectively. Table 3.1 describes the naming convention of knee forces, moments, and angles.

Table 3.1 Knee force, moment, and angle naming and direction description.

\begin{tabular}{|l|l|l|}
\hline \multicolumn{1}{|c|}{ Load } & Name & Positive Direction \\
\hline Anterior-Posterior Force & $\mathrm{F}_{\mathrm{A}-\mathrm{P}}$ & Anterior \\
\hline Medial-Lateral Force & $\mathrm{F}_{\mathrm{M}-\mathrm{L}}$ & Lateral \\
\hline Compression Force & $\mathrm{F}_{\mathrm{AX}}$ & Compression \\
\hline Varus-Valgus Moment & $\mathrm{M}_{\mathrm{V}-\mathrm{V}}$ & Valgus \\
\hline Flexion-Extension Moment & $\mathrm{M}_{\mathrm{F}-\mathrm{E}}$ & Extension \\
\hline Internal-External Rotation Moment & $\mathrm{M}_{\mathrm{IR}-\mathrm{ER}}$ & External Rotation \\
\hline Varus-Valgus Angle & V-V Angle & Valgus \\
\hline Flexion-Extension Angle & F-E Angle & Flexion \\
\hline Internal-External Rotation Angle & IR-ER Angle & Internal Rotation \\
\hline
\end{tabular}




\subsection{EXPERIMENTAL RESULTS}

The mean and standard deviation (SD) for each maximum knee force, moment, and angle are reported for each exercise and population (Mean \pm 1 SD) in table 3.2 and represented in figures 3.1 - 3.3. Figure 3.1 shows the force component results, figure 3.2 depicts the results for the moment components, while figure 3.3 compares knee angles against BMI and exercise factors. It can be observed from the figures that gait produced larger forces and moments than cycling in all components. The data also indicated OB subjects had higher forces and moments in all components than NW subjects in gait. However, this trend was not as prevalent when comparing NW and OB loads in cycling (C1 or $\mathrm{C} 2$ ). When looking at cycling data only, there is not a noticeable increase in knee joint loads between $\mathrm{C} 1$ and $\mathrm{C} 2$ as well as between NW and OB subjects. Last, knee joint angle magnitudes were comparable to each other in cycling for both BMI levels. In gait, however, the angle differences between NW and OB were accentuated. Figure 3.4 displays the averaged gait data for NW and OB subjects. Figure 3.5 shows the averaged cycling data for NW and OB at both cycling intensities (C1 and C2). 


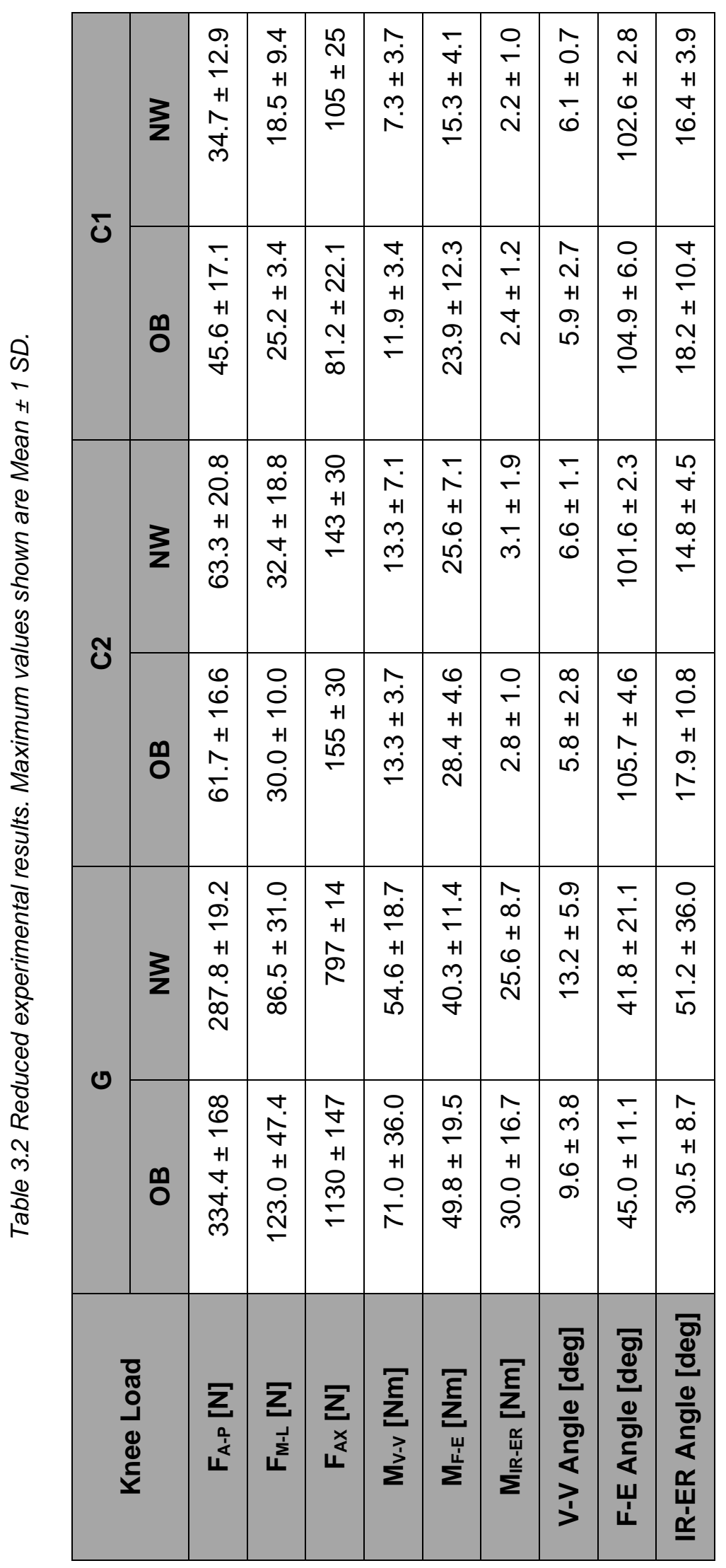



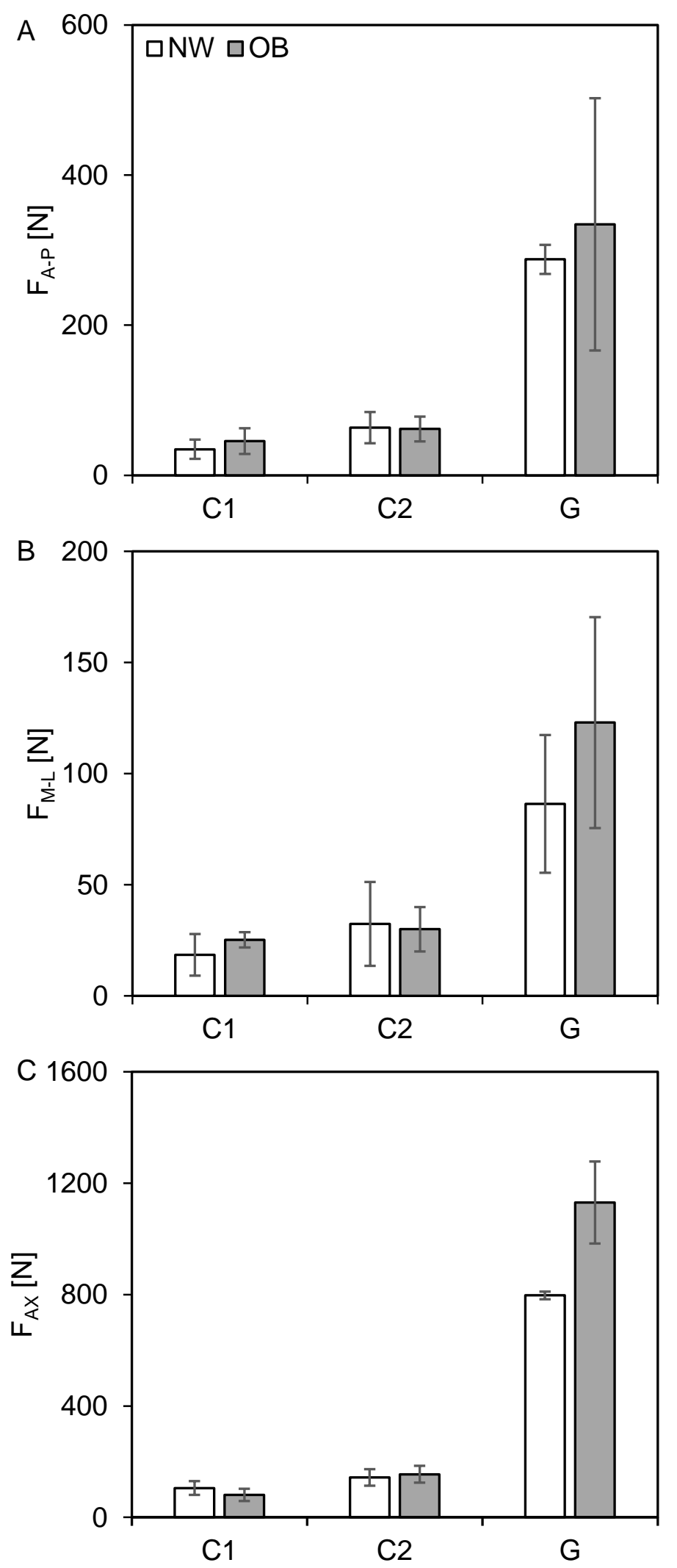

Figure 3.1 Calculated knee joint forces for normal weight and obese populations in gait and cycling. A.) Anterior force. B.) Lateral force. C.) Compressive force. Note values are mean and $1 S D$. 

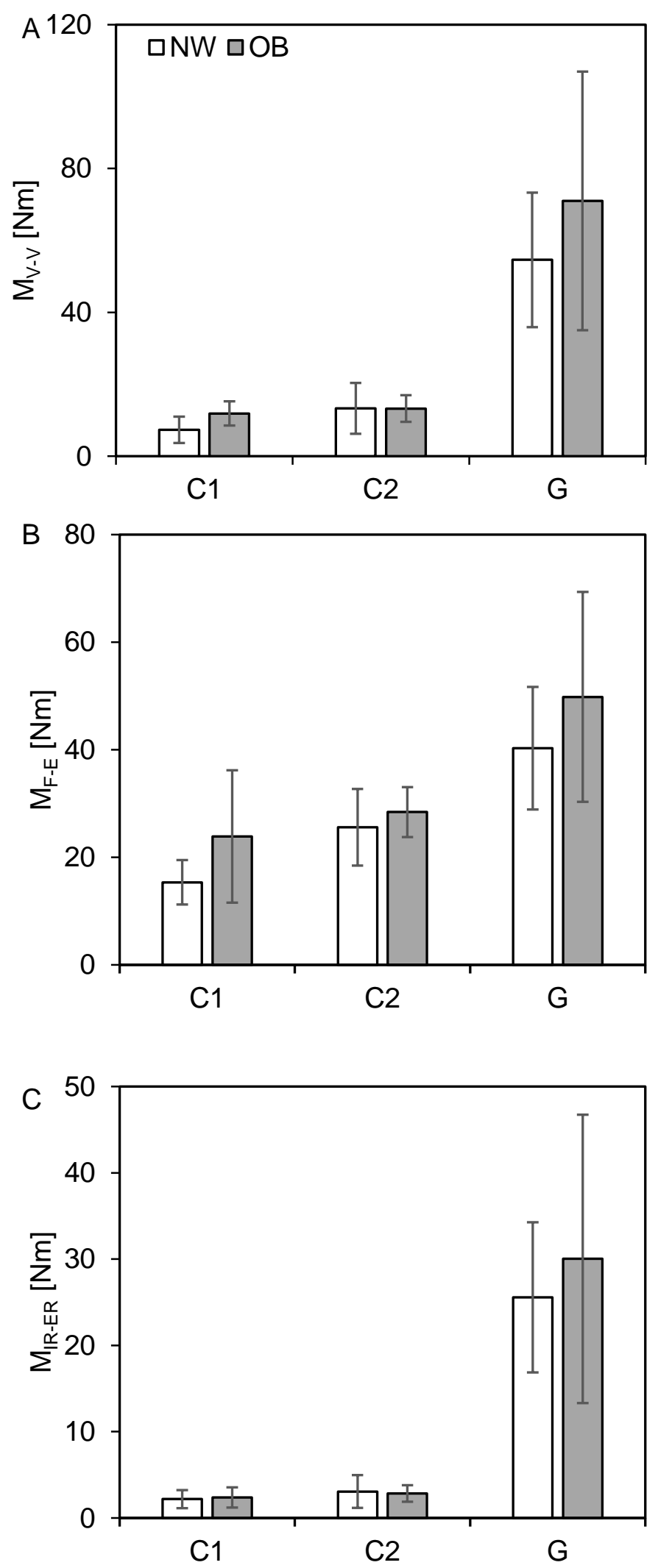

Figure 3.2 Calculated knee joint moments for normal weight and obese populations in gait and cycling. A.) Valgus moment. B.) Extension moment. C.) External rotation moment. Note values are mean and $1 S D$. 

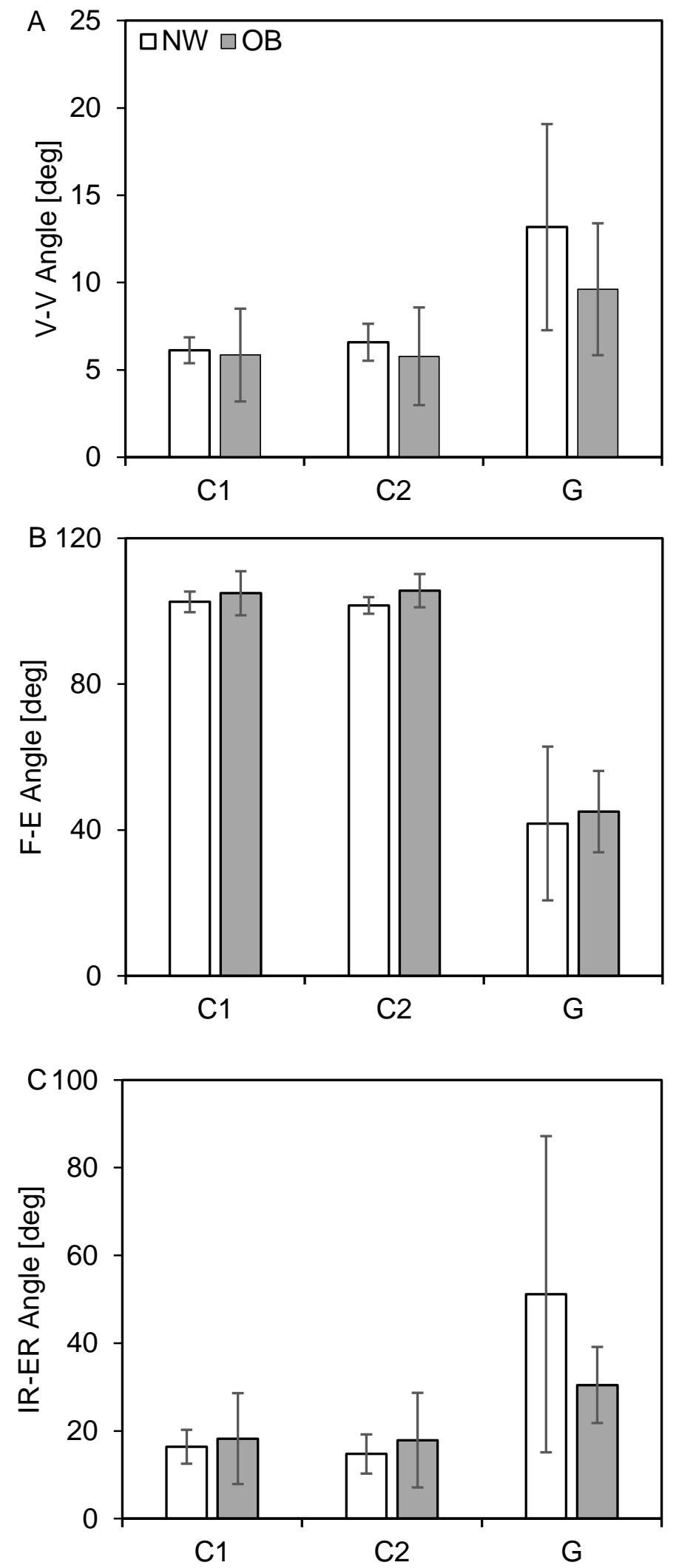

Figure 3.3 Calculated knee joint angles for normal weight and obese populations in gait and cycling. A.) Valgus angle. B.) Flexion angle. C.) Internal rotation angle. Note values are mean and $1 S D$. 


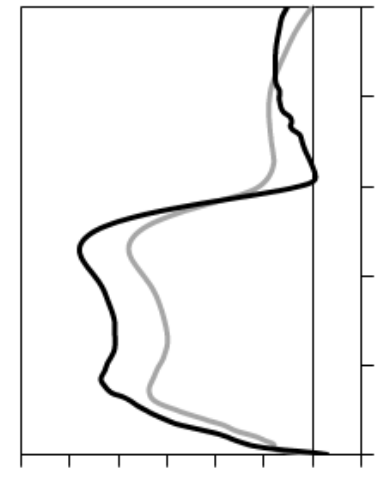

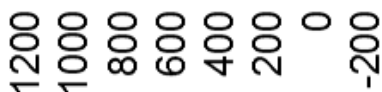
$[N]^{X \forall} y$
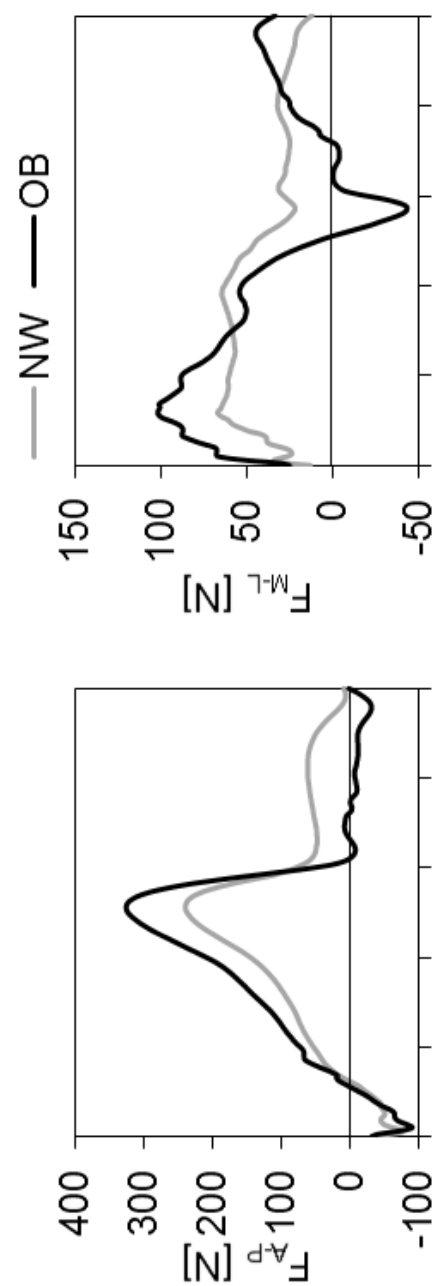
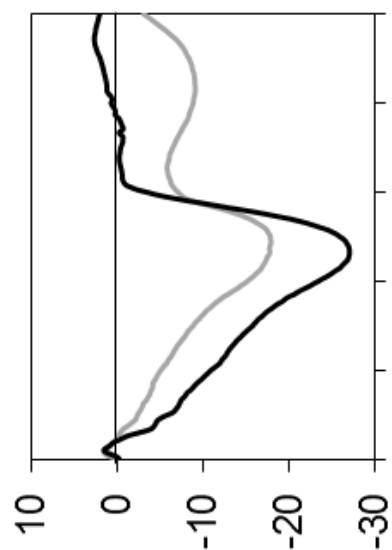

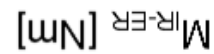
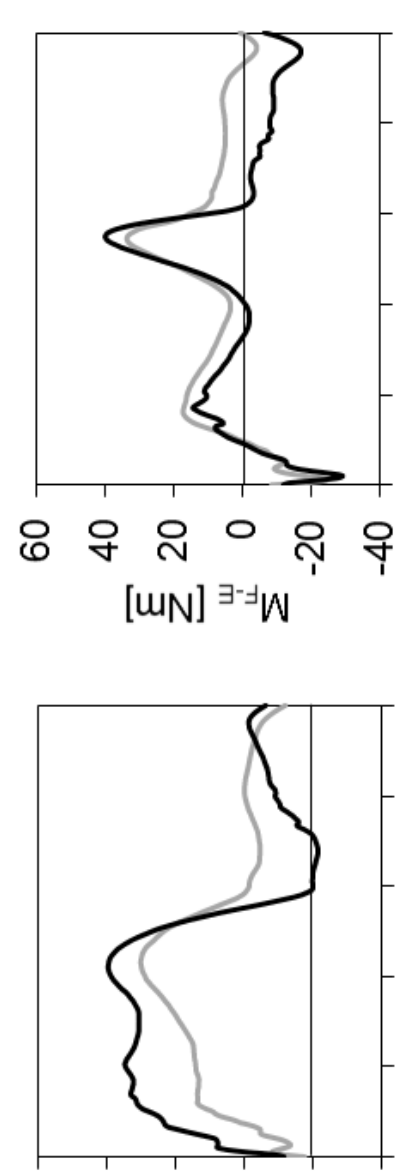

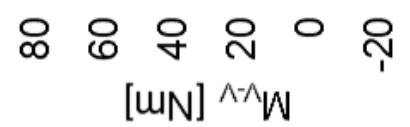

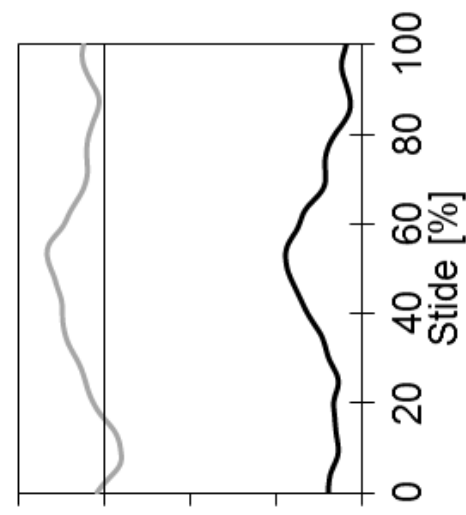

으 은 우 우

[бəр] ә|би $\forall$ y $\exists-y \mid$

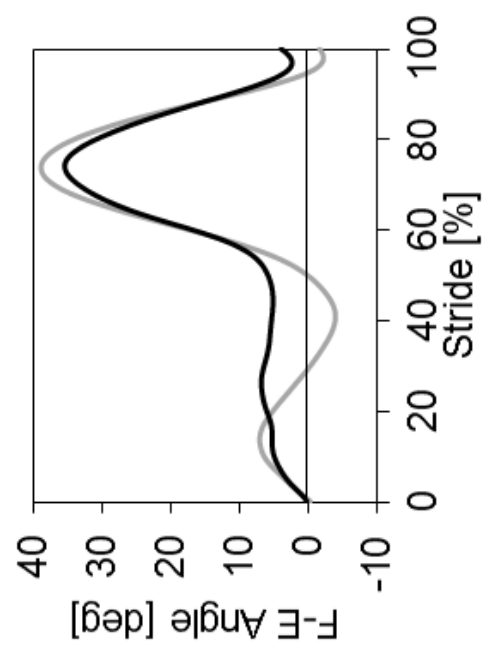

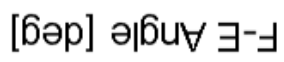

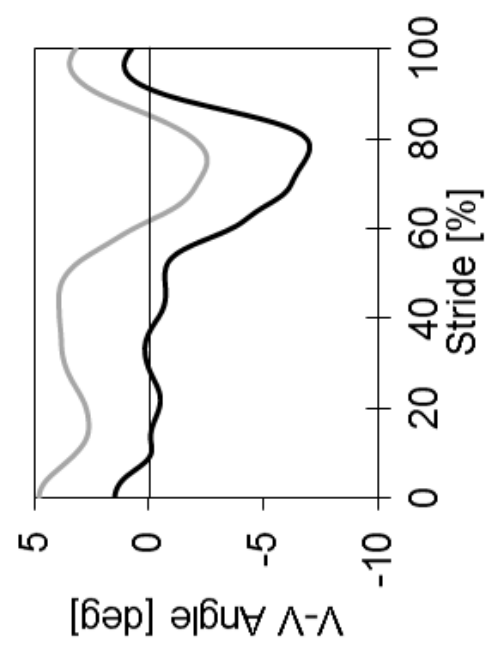

Figure 3.4 Averaged gait data for NW and OB populations. 

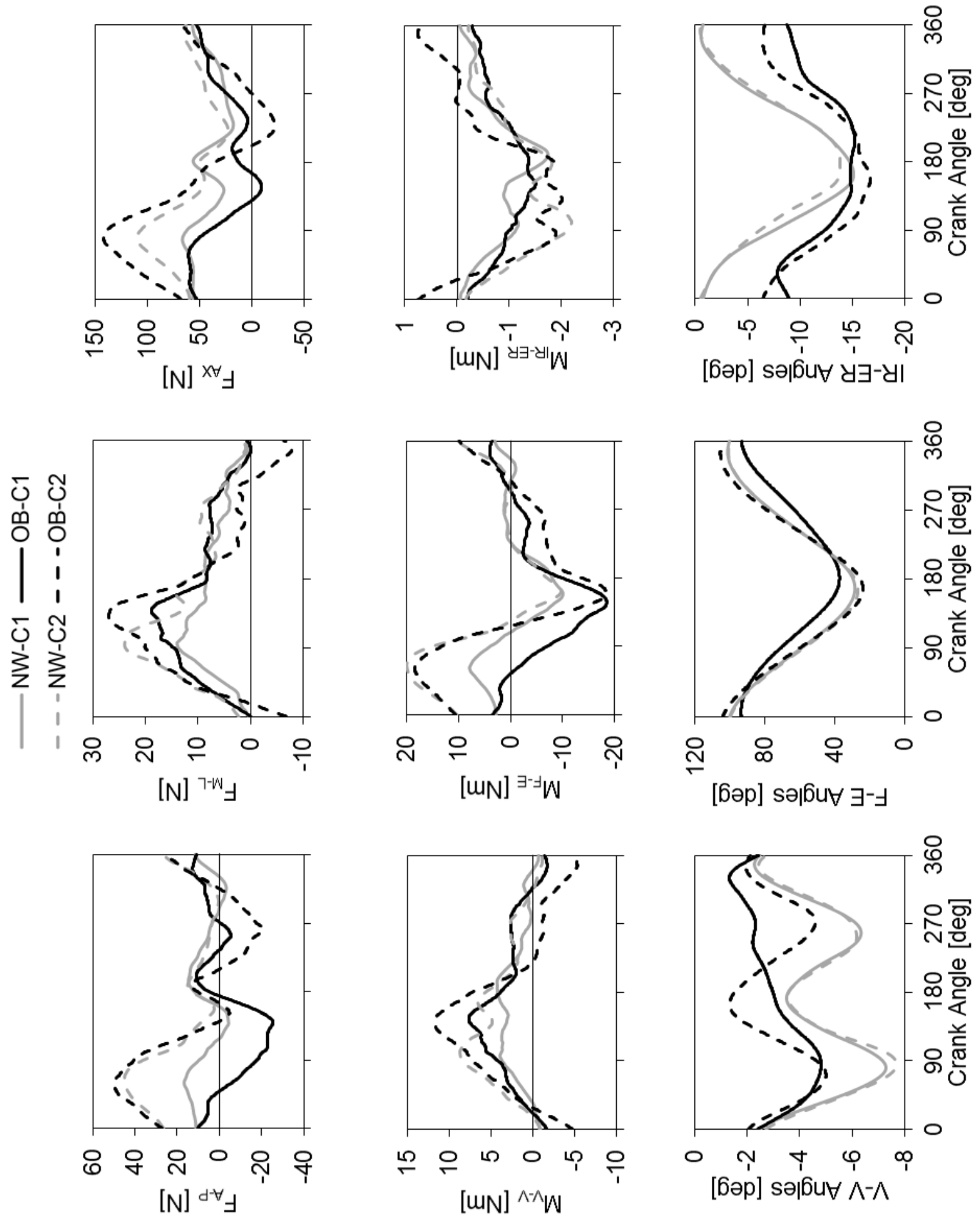

Figure 3.5 Averaged cycling data for NW and $O B$ population at both cycling intensities. 


\subsection{STATISTICAL RESULTS}

\subsubsection{ANOVA Results}

Three ANOVA tests were run. The p-values for all ANOVA test performed are listed in table 3.3. Statistical significance is indicated with an asterisk $(p<0.05)$. Marginal significance is reported with a plus sign $(0.05<p<0.10)$. The repeated measures ANOVA test only showed statistically different interactions for the vertical force component. Likewise, the one-way ANOVA test for G vs. BMI showed significance for the vertical knee force only. Meanwhile, the NW vs. Ex and OB vs. Ex one-way ANOVA tests showed significance in almost every level, meaning that the exercises performed showed statistical differences when compared to each other, regardless of BMI. Last, the C1 vs. BMI and C2 vs. BMI one-way ANOVA tests showed no significance at every level, meaning that were no statistically significant differences found in the forces, moments, and knee angles, regardless of BMI or cycling intensity.

Table 3.3 ANOVA tests p-values. BMI vs Ex shows values for the repeated measures ANOVA. All other fields represent the one-way ANOVA tests.

\begin{tabular}{|c|c|c|c|c|c|c|}
\hline $\mathbf{p}-\mathbf{v a l u e s}$ & BMI vs Ex & NW v Ex & OB v Ex & G v BMI & C1 v BMI & C2 v BMI \\
\hline $\mathbf{F}_{\text {A-P }}$ & 0.782 & $<0.001^{*}$ & $0.004^{*}$ & 0.601 & 0.346 & 0.906 \\
\hline $\mathbf{F}_{\mathrm{M}-\mathrm{L}}$ & 0.320 & $0.004^{*}$ & $0.001^{*}$ & 0.244 & 0.228 & 0.831 \\
\hline $\mathbf{F}_{\mathrm{AX}}$ & $<0.001^{*}$ & $<0.001^{*}$ & $<0.001^{*}$ & $0.004^{*}$ & 0.196 & 0.596 \\
\hline $\mathbf{M}_{\mathrm{V}-\mathrm{v}}$ & 0.633 & $0.001^{*}$ & $0.005^{*}$ & 0.450 & 0.119 & 0.986 \\
\hline $\mathbf{M}_{\mathrm{F}-\mathrm{E}}$ & 0.745 & $0.006^{*}$ & $0.053+$ & 0.433 & 0.236 & 0.529 \\
\hline $\mathbf{M}_{\text {IR-ER }}$ & 0.816 & $<0.001^{*}$ & $0.004^{*}$ & 0.653 & 0.820 & 0.845 \\
\hline V-V Angle & 0.611 & $0.033^{*}$ & 0.194 & 0.350 & 0.850 & 0.610 \\
\hline F-E Angle & 0.983 & $<0.001^{*}$ & $<0.001^{*}$ & 0.793 & 0.501 & 0.159 \\
\hline IR-ER Angle & 0.269 & $0.063+$ & 0.183 & 0.307 & 0.751 & 0.612 \\
\hline
\end{tabular}

${ }^{*}$ denotes statistically significant results $(p<0.05)$

+ indicates marginally significant results $(0.05<p<0.10)$ 


\subsubsection{Tukey Test Results}

The Tukey tests results are outlined below. Gait and cycling ( $\mathrm{C} 1$ or $\mathrm{C} 2)$ data for normal weight subjects are named NW-G and NW-C\#, respectively. Likewise, gait and cycling data for obese subjects are named OB-G and OB-C\#.

\subsubsection{Exercise vs. BMI (Repeated Measures ANOVA + Tukey Test):}

The only statistical significance observed in the repeated ANOVA test was found in the $F_{A X}$ force component $(p<0.001)$. Tukey test revealed OB-G axial knee force was larger than NW-G. Furthermore, it was found that NW-G had higher loads than all cycling trials (NW-C2, OB-C2, NW-C1, and OB-C1).

\subsubsection{NW or OB vs. Exercise (1 way ANOVA + Tukey Test):}

The Tukey test revealed that gait had larger loads than cycling in almost all load components for both NW and OB subjects $(p<0.033)$. NW-G had larger loads than NW-

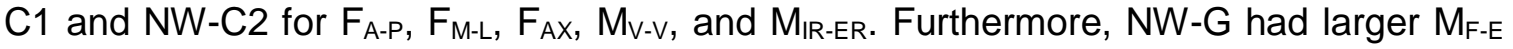
magnitude than NW-C1. Similarly, OB-G showed increased loads than OB-C1 and OB-

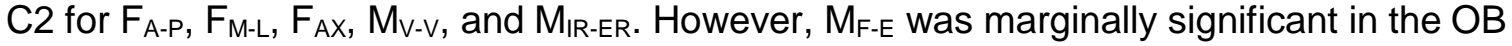
trials, suggesting larger sample sizes may show differences in this component. It is worth nothing that there are significant differences in $\mathrm{M}_{\mathrm{F}-\mathrm{E}}$ in NW but not in $\mathrm{OB}$ trials.

There were significant differences observed in knee angles. NW-C2 and NW-C1 showed larger magnitudes for F-E angles than NW-G. NW-C1 also showed larger V-V angles than NW-G. Furthermore, OB-C2 and OB-C1 had larger F-E angles than OB-G. The V-V angle significance observed in NW subjects was not found in OB subjects. There was no statistical differences among other levels, however, marginally significant results were observed in IR-ER angle for NW subjects $(p=0.063)$ and $M_{F-E}$ for OB subjects $(p=$ $0.053)$. 


\subsubsection{Gait or Cycling vs. BMI (1 way ANOVA + Tukey Test):}

There were no statistically significant differences observed in cycling (C1 or $\mathrm{C} 2)$ between $\mathrm{OB}$ and NW. Only the $\mathrm{F}_{\mathrm{Ax}}$ was significant in $\mathrm{G}$ between NW and $\mathrm{OB}$. It was observed that OB-G $>$ NW-G $(p=0.004)$.

\subsubsection{Regression Line Tests}

Figure 3.6 plots knee force components for $\mathrm{G}, \mathrm{C} 1$, and $\mathrm{C} 2$ against BMI. Figure 3.7 plots knee moment components for the same exercise factors. The $\mathrm{R}^{2}$ values are shown for all sets of data. The highest $R^{2}$ value is 0.8218 which was observed in $F_{A X}$ gait data. The p-values for the t-test analyses of the regression slopes are shown in table 3.4. The results showed that only the trend for the compression force, $F_{A X}$, for gait was significant $(p=0.002)$, i.e. the slope of the regression line of $F_{A X} v s$. BMI for $G$ is statistically different than zero. The slope of the regression line for $\mathrm{F}_{\mathrm{AX}}$ for gait is 24.8 , meaning that as $\mathrm{BMI}$ increases, the compressive load in the knee increases as well. Last, marginally significant results include $F_{M-L}$ in $G(p=0.084)$ and $M_{V-V}$ in $C 1(p=0.076)$.

Table 3.4 T-test on the slope of the regression line for Exercise vs. BMI. * denotes statistically significant results $(p<0.05)$. + denotes marginally significant results $(0.05<p<0.10)$.

\begin{tabular}{|c|c|c|c|c|c|c|}
\hline \multirow{2}{*}{ Exercise } & \multicolumn{5}{|c|}{$\mathbf{p}$-values for slope of regression line Exercise vs BMI } \\
\cline { 2 - 7 } & $\mathbf{F}_{\text {A-P }}$ & $\mathbf{F}_{\mathbf{M}-\mathrm{L}}$ & $\mathbf{F}_{\text {AX }}$ & $\mathbf{M}_{\mathrm{V}-\mathrm{V}}$ & $\mathbf{M}_{\mathrm{F}-\mathrm{E}}$ & $\mathbf{M}_{\text {IR-ER }}$ \\
\hline C1 & 0.322 & 0.168 & 0.311 & $0.076+$ & 0.248 & 0.751 \\
\hline C2 & 0.817 & 0.835 & 0.835 & 0.889 & 0.629 & 0.747 \\
\hline G & 0.450 & $0.084+$ & $0.002 *$ & 0.218 & 0.422 & 0.352 \\
\hline
\end{tabular}



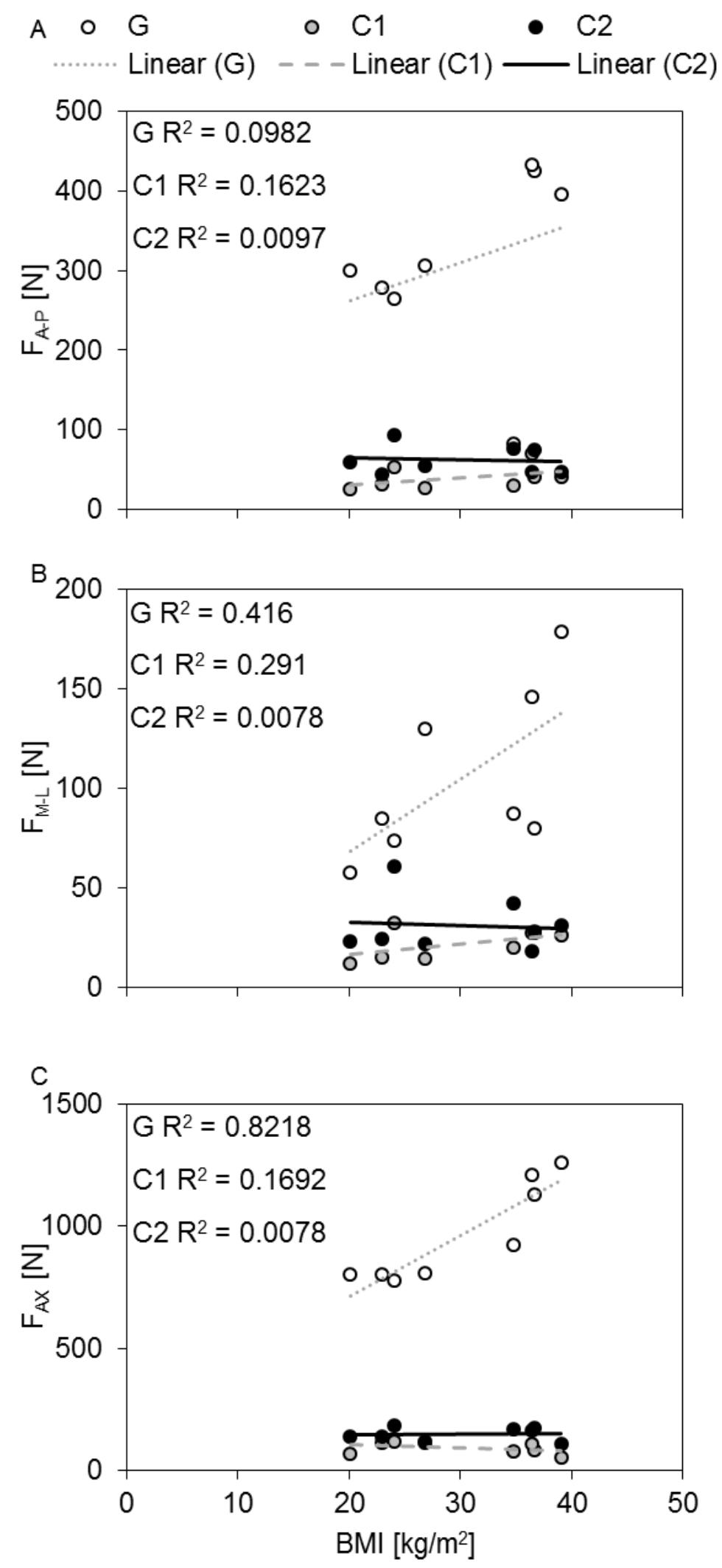

Figure 3.6 Knee forces for cycling and gait vs. BMI. A.) Anterior-Posterior force. B.) MedialLateral force. C.) Axial (compressive) force. 


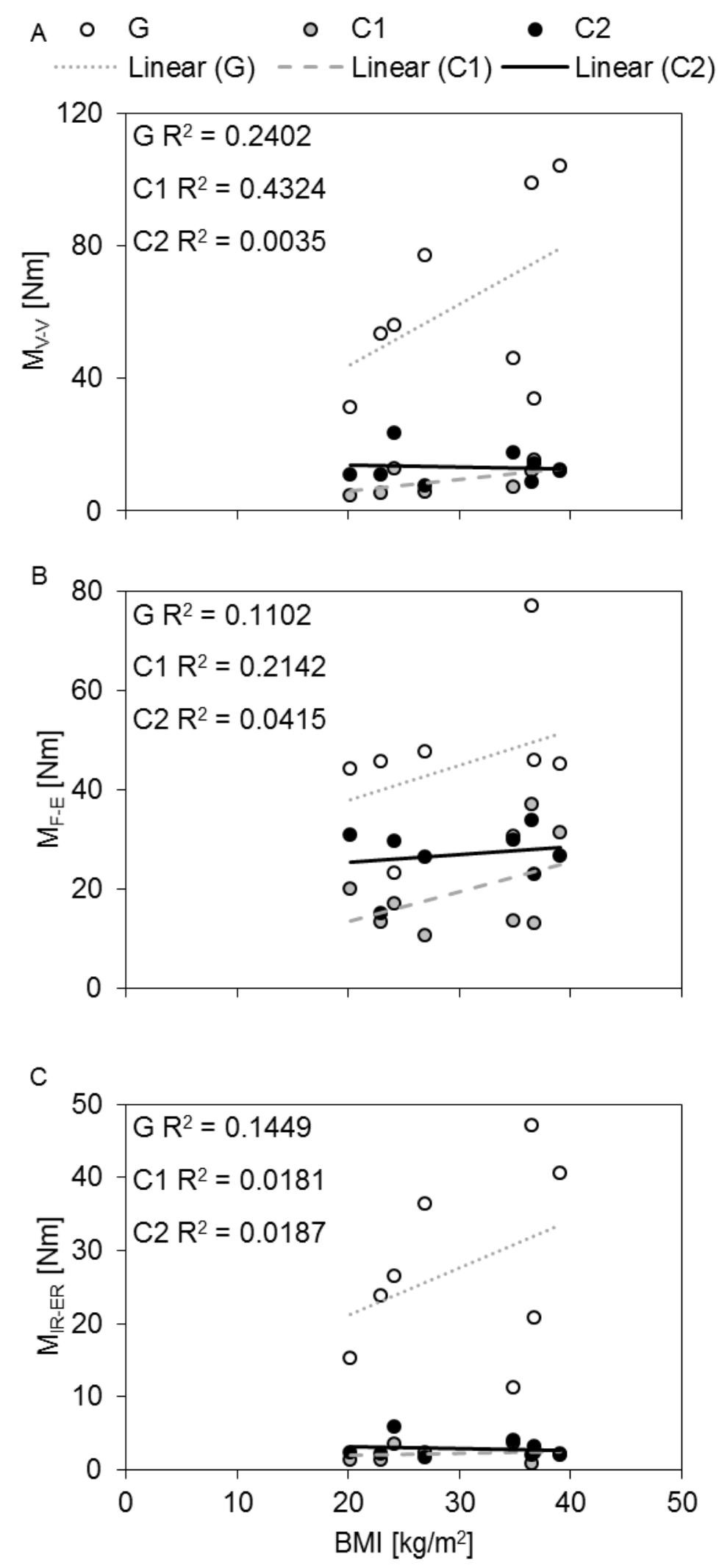

Figure 3.7 Knee moments for cycling and gait vs. BMI. A.) Varus-Valgus moment. B.) FlexionExtension moment. C.) Internal-External Rotation moment. 


\subsubsection{Power Analysis}

The ANOVA and t-test analyses showed there were some factors and interactions that were marginally significant $(0.05<p<0.10)$, suggesting a larger sample size might have shown additional statistical differences. These values were the IR-ER angle for the NW vs. Ex one-way ANOVA ( $p=0.063$ ), the MF-E for the OB vs. Ex one-way ANOVA ( $p$ $=0.053)$, and in the t-test analyses of the regression slopes, $F_{M-L}$ in gait $(p=0.084)$ and the $\mathrm{Mv}_{\mathrm{v}}$ in $\mathrm{C} 1(\mathrm{p}=0.076)$. As a last step, a power analysis was done on the cycling data to find out the sample sizes needed to see statistical differences not observed in this analysis due to having too small of a sample size. Table 3.5 and table 3.6 show the averages, SD, and sample size (SS) calculated for $\mathrm{C} 1$ and $\mathrm{C} 2$ data, respectively. For $\mathrm{C} 1$ data, the lateral and compressive force as well as the varus and extension moments have sample sizes under 14 meaning a viable experiment could be done to find these statistical

differences. For C2 data only the flexion angle has a small sample size $(n=10)$ suggesting a statistical difference may be present. 
Table 3.5 Sample size calculation results for C1 data.

\begin{tabular}{|c|r|r|r|r|r|}
\hline & \multicolumn{2}{|c|}{ NW } & \multicolumn{2}{c|}{ OB } & \multicolumn{1}{c|}{ SS } \\
\cline { 2 - 6 } & \multicolumn{1}{|c|}{ Ave } & \multicolumn{1}{c|}{ SD } & \multicolumn{1}{c|}{ Ave } & \multicolumn{1}{c|}{ SD } & \\
\hline $\mathbf{F}_{\text {A-P }}$ & 34.7 & 12.9 & 45.6 & 17.1 & 24 \\
\hline $\mathbf{F}_{\mathrm{M}-\mathrm{L}}$ & 18.5 & 9.4 & 25.2 & 3.4 & 14 \\
\hline $\mathbf{F}_{\mathrm{AX}}$ & 105.2 & 24.6 & 81.2 & 22.1 & 12 \\
\hline $\mathbf{M}_{\mathrm{V}-\mathrm{v}}$ & 7.3 & 3.7 & 11.9 & 3.4 & 7 \\
\hline $\mathbf{M}_{\mathrm{F}-\mathrm{E}}$ & 15.3 & 4.1 & 23.9 & 12.3 & 14 \\
\hline $\mathbf{M}_{\text {IR-ER }}$ & 2.2 & 1.0 & 2.4 & 1.2 & 438 \\
\hline V-V Angle & 6.1 & 0.7 & 5.9 & 2.7 & 637 \\
\hline F-E Angle & 102.6 & 2.8 & 104.9 & 6.0 & 48 \\
\hline IR-ER Angle & 16.4 & 3.9 & 18.2 & 10.4 & 224 \\
\hline
\end{tabular}

Table 3.6 Sample size calculation results for C2 data.

\begin{tabular}{|c|r|r|r|r|r|}
\hline & \multicolumn{2}{|c|}{ NW } & \multicolumn{2}{c|}{ OB } & \multicolumn{1}{c|}{ SS } \\
\cline { 2 - 6 } & \multicolumn{1}{|c|}{ Ave } & \multicolumn{1}{c|}{ SD } & \multicolumn{1}{c|}{ Ave } & \multicolumn{1}{c|}{ SD } & \\
\hline $\mathbf{F}_{\text {A-P }}$ & 63.3 & 20.8 & 61.7 & 16.6 & 1641 \\
\hline $\mathbf{F}_{\mathrm{M}-\mathrm{L}}$ & 32.4 & 18.8 & 30.0 & 10.0 & 498 \\
\hline $\mathbf{F}_{\text {AX }}$ & 143.0 & 29.6 & 154.8 & 30.4 & 79 \\
\hline $\mathbf{M}_{\mathrm{V}-\mathrm{V}}$ & 13.3 & 7.1 & 13.3 & 3.7 & 77584 \\
\hline $\mathbf{M}_{\mathrm{F}-\mathrm{E}}$ & 25.6 & 7.1 & 28.4 & 4.6 & 55 \\
\hline $\mathbf{M}_{\text {IR-ER }}$ & 3.1 & 1.9 & 2.8 & 1.0 & 592 \\
\hline V-V Angle & 6.6 & 1.1 & 5.8 & 2.8 & 86 \\
\hline F-E Angle & 101.6 & 2.3 & 105.7 & 4.6 & 10 \\
\hline IR-ER Angle & 14.8 & 4.5 & 17.9 & 10.8 & 86 \\
\hline
\end{tabular}




\section{CHAPTER 4: DISCUSSION}

\subsection{REPEATED MEASURES ANOVA}

Only the axial knee load was significant in the repeated measures ANOVA ( $p<$ 0.001). The Tukey test showed that gait had larger knee loads than cycling ( $C 1$ and $C 2)$ for OB and NW subjects. Furthermore, the axial load for OB gait was higher than NW gait. This suggests that OB subjects in gait have the highest axial knee loads, followed by NW subjects in gait, and cycling creates the lowest axial loads. Gait is a weight-bearing activity therefore a higher axial load compared to cycling was expected. Likewise, OB subjects must carry more mass than NW subjects, increasing the axial knee loads. However, finding no differences for cycling data between NW and OB subjects was not expected. This could be due to the small sample size used in this experiment. The power analysis suggested that statistically significant differences could be found in $F_{M-L}, F_{A X}, M_{V-V}$, and $M_{F-}$ E with a sample size of 14 subjects and in $F_{A-P}$ with 24 subjects for C1. Statistically significant differences could be found in F-E angle for $\mathrm{C} 2$ with only 10 subjects.

\subsection{ONE-WAY ANOVA}

\subsubsection{NW vs. Exercise}

The one-way ANOVA test found significant differences for almost every variable measured $(p<0.033)$. Tukey tests showed that G loads were higher than cycling loads at both intensities for all force components and for the valgus and external rotation moments. The extension moment had a higher magnitude for $\mathrm{G}$ when compared with $\mathrm{C} 1$ while no significant difference was observed with $\mathrm{C} 2$. The knee angle data showed G had higher valgus angles than C1. Lastly, FE angles were larger in cycling than in gait. The three knee force components and the valgus and external rotation moments were higher for $\mathrm{G}$ than cycling. This was expected as gait is a weight-bearing exercise. The extension moment showed no significant difference between $\mathrm{C} 2, \mathrm{C} 1$ and $\mathrm{G}$. 
Last, the knee flexion angles were larger in cycling than in gait. This is expected because gait does not require large flexion angles while cycling uses a larger range of flexion angles. It was also found that the valgus angle is larger for $\mathrm{G}$ than it is in $\mathrm{C} 1$. Due to the way the valgus angle (and internal rotation angle) are defined in Cortex and the limitations discussed below, the results for knee angles should be interpreted with caution.

\subsubsection{OB vs. Exercise}

Similarly to the NW vs Ex one-way ANOVA, all knee force components and the valgus and external rotation moments were larger for $G$ than cycling, regardless of cycling intensity $(p<0.005)$. The flexion angle was larger for cycling than for gait. There were no significant differences for the extension moment. The extension moment mean values for $\mathrm{C} 1, \mathrm{C} 2$, and $\mathrm{G}$ were $23.9 \mathrm{Nm}, 28.4 \mathrm{Nm}$, and $49.8 \mathrm{Nm}$, respectively. It can be seen that these values were closer to each other than in the case of $C 1$ and $G$ on the previous ANOVA test, which could cause the statistical significance to disappear. It is worth nothing that the extension moment in this ANOVA test was marginally significant for $\mathrm{G}, \mathrm{C} 1$, and C2 for OB subjects $(p=0.053)$, therefore having more subjects might bring out statistical significance.

\subsubsection{G vs. BMI}

This ANOVA test showed that OB axial gait loads were higher than NW gait loads $(p=0.004)$, confirming a portion of the findings in the repeated measures ANOVA. This difference could be due to higher body mass carried by OB subjects since gait is a weightbearing exercise. No other statistically significant results were observed. 


\subsubsection{C1 and C2 vs. BMI}

No statistically significant results were observed from these ANOVA tests. It was expected that $O B$ subjects would have higher loads during cycling than NW subjects. The lack of this trend could be due to the small sample size or to OB effects (increased mass and inertial effects) not being as dominant since the majority of the weight of the subject is rested on the seat and handle bars and not supported by the knee joint. A power analysis was done to estimate the sample size needed to find statistically significant results (see section 4.3.2).

\subsection{REGRESSION STATISTICS}

\subsubsection{T-Test on the Slope of the Regression Line}

It was expected that differences between NW and OB subjects in cycling would be observed, however, this was not the case. The only statistically significant result in the regressions test was found in $F_{A X}$ data for gait $(p=0.002)$. This suggests that $F_{A X}$ loads in gait increase as BMI increases likely due to the weight-bearing nature of the activity. This was also expected as the one-way ANOVA indicated statistically significant results at this level $(p=0.004)$. Marginally significant results were also observed in $F_{M-L}$ for $G(p=0.084)$ and $\mathrm{M}_{\mathrm{v}-\mathrm{v}}$ for $\mathrm{C} 1(\mathrm{p}=0.076)$. The corresponding one-way ANOVA tests for $\mathrm{C} 1$ and $\mathrm{G}$ did not show statistically significant or marginally significant results for these levels, however, these were the lowest non-significant $p$-vales in the test, which could suggest that larger sample sizes could reveal significant differences at these levels. The fact that $\mathrm{Mv}_{\mathrm{v}-\mathrm{v}}$ is marginally significant in $\mathrm{C} 1$ and not significant in $\mathrm{C} 2$ could be due to the cycling intensity dominating the load response and covering inertial effects in knee loads.

\subsubsection{Sample Size Power Study}

The sample size power study showed several knee loads in C1 could show statistically significant differences between NW and OB subjects if a larger sample size is 
used. These loads include $F_{A-P}$ (24 subjects), $F_{M-L}$ (14 subjects), $F_{A X}$ (12 subjects), MV-V (7 subjects), and $\mathrm{M}_{\mathrm{F}-\mathrm{E}}$ (14 subjects). The test also suggested that the F-E angle in $\mathrm{C} 2$ could exhibit statistically significant results with a sample size of 10 subjects per population (NW and $\mathrm{OB}$ ). Some of these sample sizes require sizeable resources to perform these experiments. For example $\mathrm{F}_{\mathrm{A}-\mathrm{P}}$ for $\mathrm{C} 1$ would require close to 50 subjects be tested (24 NW, $24 \mathrm{OB}$ ). Other loads not mentioned include $\mathrm{F}-\mathrm{E}$ angle in $\mathrm{C} 1$ (48 NW, $48 \mathrm{OB}$ ), and $\mathrm{F}_{\mathrm{AX}}$ (79 NW, 79 OB) and $\mathrm{M}_{\mathrm{F}-\mathrm{E}}(55 \mathrm{NW}, 55 \mathrm{OB})$ in C2. Other values required larger sample sizes, suggesting that there is not a statistically significant difference or trend in these results.

\subsection{COMPARISONS TO PUBLISHED VALUES}

\subsubsection{Gait}

Gait data was compared to two published studies. Lerner et al. explored the effects of walking speed on knee resultant loads [17]. All experimental forces and moments followed the same trend as the values published by Lerner even if the same force or moment magnitudes, or exact percent stride location was not matched. $F_{A-P}$ was mostly anterior, $F_{M-L}$ was mostly lateral, and $F_{A X}$ was compressive for all percent stride values . $F_{A-}$ $\mathrm{P}$ and $\mathrm{F}_{\mathrm{M}-\mathrm{L}}$ switch over (to posterior and medial, respectively) occurred at similar percent stride values when compared to the published values. All loads had low magnitudes after $\sim 60 \%$ stride, which was also seen in the published values. The low loads occur during the swing phase of gait. Any slight differences between experimental and published data could be attributed to the different walking speeds used by Lerner et al. and the use of normal weight subjects. Browning and Kram explored the effects of obesity in knee flexion angles at different walking speeds [5]. The maximum F-E angle occurred at about $70 \%$ stride in experimental and published data for NW and OB subjects. There was a local maximum for F-E angles at about $20 \%$ stride seen on both sets of data for NW subjects. This effect 
was not seen in experimental $O B$ data. The published values showed decreased $O B$ walking speed decreased the local maximum at $20 \%$ stride until it is almost gone at about $0.5 \mathrm{~m} / \mathrm{s}$. Any differences in the published values with experimental results could be due to the different walking speeds used in the experiments. Browning and Kram used a walking speed range of $0.5 \mathrm{~m} / \mathrm{s}$ to $1.75 \mathrm{~m} / \mathrm{s}$, while this study used self-selected walking speeds. The average NW self-selected gait speed was $1.17 \pm 0.10 \mathrm{~m} / \mathrm{s}$, while average gait OB self-selected speed was $1.17 \pm 0.05 \mathrm{~m} / \mathrm{s}$ (Mean $\pm 1 \mathrm{SD}$ ).

\subsubsection{Cycling}

Cycling data was compared to data published by Ruby et al. [13]. It was noted that all cycling knee forces and moments increased at around the same crank angle values, maintaining a similar shape throughout the crank angle range. $F_{A-P}, F_{M-L}$, and $F_{A X}$ had maximum loads between 70 and 90 degrees crank angle and minimum values at around 200-250 degrees crank angle. This trend was seen in the published data though the magnitude of the peaks was larger in published data and the crank angles for these peaks did not match experimental results exactly. These differences could be explained by the sample size, rider weight, and rider experience. This study used 4 NW and 4 OB subjects whose riding experience was defined as amateur. Meanwhile, Ruby et al. used 11 NW subjects and rider experience ranged from commuter to Category 3 racer [13]. The experienced riders may increase the peak values for each knee load and ride different to amateurs which could create maximum and minimum peaks at different crank angles. 


\subsection{LIMITATIONS}

This study is limited by several factors. Due to this care must be taken when analyzing the results obtained.

\subsubsection{Soft Tissue Artifact}

The knee joint load calculations assume that the markers track bone position which is modeled as a rigid body. However, the markers are placed on top of muscle and adipose tissue, whose movement may differ from the true path of the bone (rigid body). This effect is referred to as soft tissue artifact (STA) and is a primary source of error that propagates in the knee joint load results [18] [19] [20]. This study did not correct for STA. A few ways have been proposed to deal with STA. Benoit et al. proposed a standard error of measurement to compensate for STA errors in marker position [18]. Andriacchi et al. developed a cluster method to reduce the effect of STA in kinematic data [19].

\subsubsection{Marker Set Placement}

The Helen Hayes marker set was used to measure knee angles. Misalignment of markers on anatomical landmarks may introduce crosstalk between knee angles. Figure 3.4 shows an increase in gait $\mathrm{V}-\mathrm{V}$ and $\mathrm{F}-\mathrm{E}$ angle magnitudes at around $70 \%$ to $80 \%$ stride. Figure 3.5 shows a synchronized increase in magnitude for all knee angles during cycling at a crank angles close to 180 degrees. Knee axes misalignment could cause parts of the larger F-E angles to be recorded as $\mathrm{V}-\mathrm{V}$ or IR-ER, increasing these angles at the same crank angle as the large F-E angles occur. While knee F-E angle has a large range of motion, $\mathrm{V}-\mathrm{V}$ and IR-ER angles have a smaller range of motion which makes them more susceptible to marker position error. This error could come from the accuracy of the motion analysis cameras or from the accuracy of the marker placement on anatomical landmarks. For instance, the definition of the knee joint axes has a large effect on the $\mathrm{V}-\mathrm{V}$ angle and has been attributed to the variation in V-V angle values in the literature [21]. Misplacement 
of knee markers combined with errors in marker position due to camera accuracy change the definition of the knee joint axes in Cortex which in turn changes the calculated V-V and IR-ER angles. The poor accuracy in V-V and IR-ER knee angles could explain the 25 degree difference in cycling IR-ER angles. For these reasons, care must be taken when analyzing the knee angle results as the measurements are not reliable enough to confidently measure V-V and IR-ER knee joint angles.

Marker placement errors in $\mathrm{OB}$ subjects are likely incremented due to increased adipose tissue, especially in the abdominal area, which made anatomical landmark location challenging. Also, once an anatomical position is found, the adipose tissue makes the marker be placed farther away from that landmark than in NW subjects which could change subject modeling and calculated results in Cortex. The ASIS markers are the most affected by these issues. Last, the adipose tissue increases the effect of STA in OB subjects. There were not corrections for these effects in this study.

\subsubsection{Resultant Load vs. Joint Contact Force}

Cortex calculates three force components and three moment components. These resultant loads are the forces and moments needed at the knee joint to balance the force and moment equations described in Appendix B. These equations use Ground Reaction Forces (GRF) and kinematic data only. Resultant forces differ from joint contact loads, or the load seen by the cartilage tissue. To calculate joint contact force the muscle forces must be included in the analysis. Muscle forces are the largest contributors to the joint contact force between the femur and the tibia [22]. In order to accurately determine the effect of BMl and Exercise on the cartilage tissue, the joint contact force must be estimated, not the resultant knee loads. This can and should be done in future studies using OpenSim static optimization solver, possibly using EMG-driven analyses [23]. 
Electromyography (EMG) is often used in biomechanics experiments because of the relationship between muscle EMG and muscle tension. EMG can give information in muscle activation and is the primary signal to describe muscular system input [24]. EMG was not used in this study. The use of EMG data could improve the modeling done by Cortex by introducing muscle forces. Besides GRF and inertial effects, muscle forces increase the compressive knee loads. EMG data can improve the accuracy of the estimated knee joint contact force by accounting for which muscles are activated and estimating the force applied by each of them.

\subsection{CONCLUSIONS}

The specific objectives of this study were to determine if (1) cycling produces lower knee resultant loads when compared to gait for normal weight and obese subjects, (2) obese subjects produce higher knee resultant loads than normal weight subjects while cycling, and (3) obese subjects have higher knee resultant loads than normal weight individuals in gait. This study's objectives were aimed at determining if cycling is a preferred weight-loss exercise to walking due to decreased knee resultant loads which may reduce the risk of developing knee OA. It was found that cycling, even at moderate intensities, has lower resultant load magnitudes than gait for normal weight and obese subjects. Also, it was found that obese subjects have higher axial knee loads than normal weight subjects for gait. Finally, the results suggest there are no differences in knee resultant loads between normal weight and obese subjects in cycling. When comparing cycling and gait as potential weight control exercises, cycling produced lower knee loads for both NW and OB subjects and, furthermore, cycling substantially reduced or eliminated differences in knee loads observed between NW and OB subjects (as observed in gait) thereby restoring $\mathrm{OB}$ knee biomechanics to normal levels. In conclusion, the results suggest cycling to be a preferred weight-loss exercise (as compared to walking) for obese 
subjects as knee resultant forces and moments are lower, but more work needs to be done to address the limitations, especially correcting for STA, calculating joint contact loads instead of joint resultant loads, and increasing the sample size. 


\section{REFERENCES}

[1] L. A. Setton, D. M. Elliot and V. C. Mow, "Altered mechanics of cartilage with osteoarthritis: human osteoarthritis and an experimental model of joint degeneration," Osteoarthritis and Cartilage, vol. 7, no. 1, pp. 2-14, 1999.

[2] D. T. Felson, "Weight and osteoarthritis," The American Journal of Clinical Nutrition, vol. 63, no. 3, pp. 430S-2S, 1996.

[3] M. R. Sowes and C. A. Karvonen-Gutierrez, "The evolving role of obesity in knee osteoarthritis," Curr Opin Rheumatol, vol. 22, no. 5, pp. 533-7, 2010.

[4] T. Andriacchi and M. Annegret, "The role of ambulatory mechanics in the initiation and progression of knee osteoarthritis," Curr Opin Rheumatol., vol. 18, no. 5, pp. 514-8, 2006.

[5] R. C. Browning and R. Kram, "Effects of Obesity on the Biomechanics of Walking at Different Speeds," Medicine and Science in Sports and Exercise, vol. 39, no. 9, pp. 1632-41, 2007.

[6] S. P. Messier, D. J. Gutekunst, C. Davis and P. DeVita, "Weight Loss Reduces Knee-Joint Loads in Overweight and Obese Older Adults With Knee Osteoarthritis.," Arthritis \& Rheumatism, vol. 52, no. 7, pp. 2026-32, July 2005.

[7] R. Lee and W. F. Kean, "Obesity and Knee Osteoarthritis," Inflammopharmacol, vol. 20, pp. 53-8, 2012. 
[8] L. Sharma, C. Lou, S. Cahue and D. D. Dunlop, "The Mechanism of the Effect of Obesity in Knee Osteoarthritis," Arthritis \& Rheumatism, vol. 43, no. 3, pp. 568-75, 2000.

[9] S. P. Messier, "Osteoarthritis of the knee and associated factors of age and obesity: effects on gait," Med Sci Sports Exerc., vol. 26, no. 12, pp. 1446-52, 1994.

[10] W. L. Haskell, I.-M. Lee, R. R. Pate, K. E. Powell, S. N. Blair, B. A. Franklin, C. A. Macera, G. W. Heath, P. D. Thompson and A. Bauman, "Physical Activity and Public Health: Updated Recommendation for Adults From the American College of Sports Medicine and the American Heart Association," Circulation, vol. 116, no. 9, pp. 1081-93, 2007.

[11] M. Ericson, "On the biomechanics of cycling. A study of joint and muscle load during exercise on the bicycle ergometer.," Scand J Rehabil Med Suppl, vol. 16, pp. 1-43, 1986.

[12] M. L. Hull and R. R. Davis, "Measurement of Pedal Loading in Bicycling: I. Instrumentation," J. Biomechanics, vol. 14, no. 12, pp. 843-56, 1981.

[13] P. Ruby, M. L. Hull and D. Hawkins, "Three-Dimensional Knee Joint Loading During Seated Cycling," J. Biomechanics, vol. 25, no. 1, pp. 41-53, 1992.

[14] C. Stone and M. L. Hull, "The Effect of Rider Weight on Rider-Induced Loads During Common Cycling Situations," J. Biomechanics, vol. 28, no. 4, pp. 365-75, 1995.

[15] D. C. Kerrigan, M. K. Todd and U. D. Croce, "Gender Differences in Joint Biomechanics During Walking Normative Study in Young Adults.," American Journal of Physical Medicine \& Rehabilitation, vol. 77, no. 1, 1998. 
[16] "Sample Size Calculators," DSS Research, [Online]. Available: https://www.dssresearch.com/KnowledgeCenter/toolkitcalculators/samplesizecalc ulators.aspx. [Accessed 23 February 2015].

[17] Z. F. Lerner, D. J. Haight, M. S. Demers, W. J. Board and R. C. Browning, "The Effects of Walking Speed on Tibiofemoral Loading Estimated Via Musculoskeletal Modeling," J Appl Biomech., vol. 30, no. 2, pp. 197-205, 2014.

[18] D. L. Benoit, D. K. Ramsey, M. Lamontagne, L. Xu, P. Wretenberg and P. Renstrom, "Effect of skin movement artifact on knee kinematics during gait and cutting motions measured in vivo.," Gait \& Posture, vol. 24, pp. 152-64, 2006.

[19] T. P. Andriacchi, E. J. Alexander, M. K. Toney, C. Dyrby and J. Sum, "A Point Cluster Method for In Vivo Motion Analysis: Applied to a Study of Knee Kinematics," Journal of Biomechanical Engineering, vol. 120, no. 6, pp. 743-9, 1998.

[20] J. P. Holden, J. A. Orsini, K. L. Siegel, T. M. Kepple, L. H. Gerber and S. J. Stanhope, "Surface movement errors in shank kinematics and knee kinetics during gait.," Gait \& Posture, vol. 5, pp. 217-27, 1997.

[21] M. P. Kadaba, H. K. Ramakrishnan and M. E. Wootten, "Measurement of Lower Extremity Kinematics During Level Walking.," Journal of Orthopaedic Research, vol. 8, no. 3, pp. 383-92, 1990.

[22] K. B. Shelburne, M. R. Torry and M. G. Pandy, "Contributions of Muscles, Ligaments, and the Ground-Reaction Force to Tibiofemoral Joint Load During Normal Gait," Journal of Orthopaedic Research, vol. 24, no. 10, pp. 1983-90, 2006. 
[23] S. L. Delp, F. C. Anderson, A. S. Arnold, P. Loan, A. Habib, C. T. John, E. Guendelman and D. G. Thelen, "OpenSim: Open-Source Software to Create and Analyze Dynamics Simulations of Movement," IEEE Transactions on Biomedical Engineering, vol. 54, no. 11, pp. 1940-50, 2007.

[24] D. A. Winter, Biomechanics and Motor Control of Human Movement, 4th ed., Hoboken, New Jersey: John Wiley \& Sons, Inc., 2009.

[25] M. Analysis, Cortex 5.0 Reference Manual, 2013.

[26] Motion Analysis, KinTools RT User's Manual, 2013.

[27] T. E. ToolBox, "Alluminum Alloys - Mechanical Properties," [Online]. Available: http://www.engineeringtoolbox.com/properties-aluminum-pipe-d_1340.html. [Accessed 2015].

[28] McMaster-Carr, "Multipupose 6061 Aluminum," [Online]. Available: http://www.mcmaster.com/\#standard-aluminum-sheets/=12ndafd. [Accessed 2015].

[29] R. R. Davis and M. L. Hull, "Measurement of Pedal Loading in Bicycling: II. Analysis and Results," J. Biomechanics, vol. 14, no. 12, pp. 857-72, 1981.

[30] R. J. Gregor, P. R. Cavanagh and M. LaFortune, "Knee Flexor Moments During Propulsion in Cycling-A Creative Solution to Lombard's Paradox.," J. Biomechanics, vol. 18, no. 5, pp. 307-16, 1985. 
[31] P. Ruby, M. L. Hull, K. A. Kirby and D. W. Jenkins, "The Effect of Lower-Limb Anatomy on Knee Loads During Seated Cycling," J. Biomechanics, vol. 25, no. 10, pp. 1195-207, 1992.

[32] R. Redfield and M. L. Hull, "On the Relation Between Joint Moments and Pedaling Rates at Constant Power in Bicycling," J. Biomechanics, vol. 19, no. 4, pp. 317-29, 1986.

[33] M. L. Hull and M. Jorge, "A Method for Biomechanical Analysis of Bicycle Pedalling," J. Biomechanics, vol. 18, no. 9, pp. 631-44, 1985.

[34] M. Jorge and M. L. Hull, "Analysis of EMG Measurements During Bicycle Pedalling," J. Biomechanics, vol. 19, no. 9, pp. 683-94, 1986.

[35] R. J. Gregor, J. P. Broker and M. M. Ryan, "The biomechanics of cycling.," Exerc Sport Sci Rev., vol. 19, pp. 127-69, 1991.

[36] D. J. Haight, Z. F. Lerner, W. J. Board and R. C. Browning, "A Comparison of Slow, Uphill and Fast, Level Walking on Lower Extremity Biomechanics and Tibiofemoral Joint Loading in Obese and Nonobese Adults," Journal of Orthopaedic Research, vol. 32, no. 2, pp. 324-30, 2014.

[37] T. F. Boyd, R. R. Neptune and M. L. Hull, "Pedal and Knee Loads Using a MultiDegree-of-Freedom Pedal Platform in Cycling," J. Biomechanics, vol. 30, no. 5, pp. 505-11, 1997.

[38] C. S. Gregersen and M. L. Hull, "Non-driving intersegmental knee moments in cycling computed using a model that includes three-dimensional kinematics of the 
shank/foot and the effect of simplifying assumptions," Journal of Biomechanics, vol. 36, no. 6, pp. 803-13, 2003.

[39] B. J. Fregly, F. E. Zajac and C. A. Dairaghi, "Crank inertial load has little effect on steady-state pedaling coordination," J. Biomechanics, vol. 29, no. 12, pp. 1559-67, 1996.

[40] R. F. Reiser II, M. L. Peterson and J. P. Broker, "Instrumented bicycle pedals for dynamic measurement of propulsive cycling loads," Sports Engineering, vol. 6, pp. 41-8, 2003.

[41] S. A. Kautz and M. L. Hull, "A theoretical basis for interpreting the force applied to the pedal in cycling," J. Biomechanics, vol. 26, no. 2, pp. 155-65, 1993.

[42] N. Zheng, G. S. Fleisig, R. F. Escamilla and S. W. Barrentine, "An analytical model of the knee for estimation of internal forces during exercise," Journal of Biomechanics, vol. 31, no. 10, pp. 963-7, 1998.

[43] J. Velotta, J. Weyer, A. Ramirez, J. Winstead and R. Bahamonde, "Relationship between leg dominance test and type of task," Portuguese Journal of Sport Sciences, vol. 11, no. Suppl. 2, pp. 1035-8, 2011.

[44] A. G. Schneiders, S. J. Sullivan, K. J. O'Malley, S. V. Clarke, S. A. Knappstein and L. J. Taylor, "A valid and reliable clinical determination of footedness," PM\&R, vol. 2, no. 9, pp. 835-41, 2010. 
A: EXPERIMENTAL DATA

\begin{tabular}{|c|c|c|c|c|c|c|c|c|c|c|}
\hline \multirow{3}{*}{$\sum_{z}^{+}$} & $\overline{0}$ & $\begin{array}{l}\dot{\sim} \\
\stackrel{\sim}{ }\end{array}$ & $\stackrel{\text { Q }}{\mp}$ & $\begin{array}{l}\forall \\
\varnothing \\
0\end{array}$ & $\stackrel{\leftrightarrow}{\forall}$ & $\begin{array}{l}\circ \\
\stackrel{\sim}{\circ}\end{array}$ & $\stackrel{\nabla}{\sim}$ & $\stackrel{\circ}{r}$ & $\begin{array}{l}\text { ㅇ } \\
\text { ㅇ }\end{array}$ & ì \\
\hline & ঠ & \begin{tabular}{l}
$\Upsilon$ \\
\hdashline
\end{tabular} & $\overline{\mathfrak{N}}$ & $\begin{array}{l}0 \\
\text { m } \\
\end{array}$ & $\frac{\circ}{\check{r}}$ & $\begin{array}{l}\text { क् } \\
\text { ஜे }\end{array}$ & $\stackrel{+}{\sim}$ & $\stackrel{\mathscr{C}}{N}$ & $\begin{array}{l}\text { ம } \\
\text { बे }\end{array}$ & 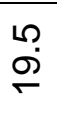 \\
\hline & 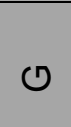 & 今̊ & مَم & 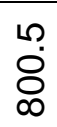 & $\frac{m}{m}$ & $\begin{array}{l}\text { Pִ } \\
\dot{y}\end{array}$ & 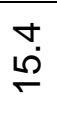 & $\bar{\infty}$ & $\begin{array}{l}\infty \\
\dot{\forall} \\
\end{array}$ & $\frac{\dot{v}}{\mathrm{~N}}$ \\
\hline \multirow{3}{*}{$\sum_{z}^{m}$} & $\overline{0}$ & $\begin{array}{l}\infty \\
\text { లై }\end{array}$ & 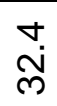 & 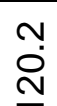 & $\stackrel{\infty}{\stackrel{\infty}{\sim}} \underset{\sim}{ }$ & $\stackrel{N}{N}$ & $\stackrel{\mathscr{\varphi}}{\oplus}$ & $\begin{array}{l}n \\
10\end{array}$ & $\begin{array}{l}\infty \\
\dot{\theta} \\
\varnothing\end{array}$ & $\stackrel{\nabla}{\leftarrow}$ \\
\hline & তิ & $\begin{array}{l}\text { 乃े } \\
\text { ஸे }\end{array}$ & $\begin{array}{l}0 \\
0 \\
0\end{array}$ & $\begin{array}{l}\dot{\forall} \\
\stackrel{\infty}{ }\end{array}$ & ヘָ & స్ & $\begin{array}{l}\text { هి } \\
0\end{array}$ & $\bar{\sigma}$ & $\begin{array}{l}\text { O) } \\
\text { ঠ் }\end{array}$ & $\stackrel{\circ}{\circ}$ \\
\hline & ৫ & $\begin{array}{l}\text { @ } \\
\text { @̊ } \\
\stackrel{N}{N}\end{array}$ & $\stackrel{+}{\sim}$ & $\stackrel{\circ}{N}$ & 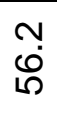 & ભై & $\begin{array}{l}\text { مٌ } \\
\stackrel{N}{ }\end{array}$ & $\begin{array}{l}\infty \\
\sigma^{\prime}\end{array}$ & $\frac{\text { क }}{\text { in }}$ & $\frac{0}{m}$ \\
\hline \multirow{3}{*}{$\sum_{\mathbf{z}}^{\mathrm{N}}$} & $\overline{0}$ & N̦ & $\begin{array}{l}0 \\
\underset{\sim}{+}\end{array}$ & $\stackrel{\check{N}}{\check{\tau}}$ & ○् & $\hat{O}$ & $\stackrel{+}{\sim}$ & iم & $\begin{array}{l}0 \\
\text { 으 }\end{array}$ & $\stackrel{\sigma}{\sigma}$ \\
\hline & $\widetilde{\mho}$ & $\begin{array}{l}0 \\
1 \\
1\end{array}$ & $\frac{0}{\grave{N}}$ & $\stackrel{\circ}{\underset{ \pm}{ \pm}}$ & $\stackrel{\varphi}{r}$ & $\begin{array}{l}0 \\
\stackrel{0}{N} \\
\stackrel{0}{0}\end{array}$ & $\stackrel{\infty}{r}$ & $\check{\Gamma}$ & $\frac{0}{5}$ & 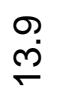 \\
\hline & ৫ & $\begin{array}{l}\mathscr{0} \\
\dot{\rho}\end{array}$ & $\stackrel{\text { N }}{\stackrel{N}{N}}$ & $\begin{array}{l}\forall \\
\infty \\
\infty \\
\infty\end{array}$ & $\stackrel{\Gamma}{N}$ & $\stackrel{\infty}{\stackrel{\infty}{f}}$ & 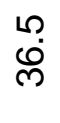 & $\frac{\dot{v}}{\mathrm{~N}}$ & $\begin{array}{l}\text { n̊ } \\
\stackrel{0}{\circ}\end{array}$ & $\begin{array}{l}\stackrel{+}{~} \\
\text { ㅇ }\end{array}$ \\
\hline \multirow{3}{*}{$\sum_{z}^{\Sigma}$} & $\overline{0}$ & $\frac{N}{m}$ & 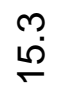 & & $\begin{array}{l}0 \\
0\end{array}$ & $\stackrel{\sim}{\stackrel{n}{m}}$ & $\stackrel{\nabla}{\sim}$ & مִ & 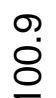 & $\stackrel{\sim}{\stackrel{D}{D}}$ \\
\hline & ত̃ & $\begin{array}{l}\stackrel{2}{8} \\
\dot{y}\end{array}$ & $\stackrel{n}{\stackrel{N}{N}}$ & 吕 & $\stackrel{0}{\mp}$ & $\begin{array}{l}m \\
\stackrel{n}{\sigma}\end{array}$ & $\stackrel{\sim}{\sim}$ & مُ & ه্ & $\begin{array}{l}0 \\
\ddot{\theta}\end{array}$ \\
\hline & ৫ & $\begin{array}{l}0 \\
\infty \\
\stackrel{\infty}{N}\end{array}$ & $\stackrel{\Gamma}{\infty}$ & $\begin{array}{l}\text { ণิ } \\
\text { ণิ }\end{array}$ & $\hat{\tilde{D}}$ & $\begin{array}{l}\infty \\
\stackrel{\rho}{\forall}\end{array}$ & ભ) & $\stackrel{\vec{m}}{\sigma}$ & $\begin{array}{l}0 \\
\text { ○ी }\end{array}$ & ণ্ \\
\hline 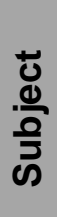 & $\begin{array}{l}0 \\
\frac{\mathscr{D}}{0} \\
\frac{0}{0} \\
\dot{x}\end{array}$ & $\stackrel{0}{\frac{0}{\alpha}}^{\frac{0}{2}}$ & $\underset{L}{\stackrel{4}{L}}$ & $\underset{\leftarrow}{\stackrel{x}{4}}$ & $\sum_{\Sigma}^{3}$ & 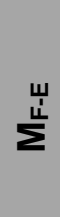 & 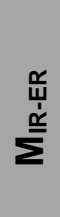 & $\begin{array}{l}\frac{0}{0} \\
\frac{2}{2} \\
⿱ 亠 乂 \\
>\end{array}$ & $\begin{array}{l}\frac{0}{\sigma} \\
\frac{\mathbf{C}}{\mathbf{\alpha}} \\
\text { 山' }\end{array}$ & $\begin{array}{l}\frac{\Phi}{\sigma} \\
\frac{0}{\mathbf{\alpha}} \\
\text { 品 } \\
\text { வ }\end{array}$ \\
\hline
\end{tabular}




\begin{tabular}{|c|c|c|c|c|c|c|c|c|c|c|}
\hline \multirow{3}{*}{ 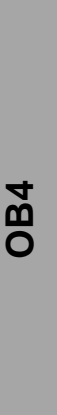 } & $\overline{0}$ & $\begin{array}{l}\infty \\
\stackrel{\infty}{0} \\
\text {. }\end{array}$ & $\check{\grave{N}}$ & $\begin{array}{l}\hat{0} \\
\text { o }\end{array}$ & $\stackrel{n}{N}$ & $\stackrel{\varphi}{\stackrel{\rho}{\sigma}}$ & $\begin{array}{l}\infty \\
\dot{m}\end{array}$ & $\begin{array}{l}\infty \\
0 \\
0\end{array}$ & 옹. & $\stackrel{\circ}{N}$ \\
\hline & ల్ & $\begin{array}{l}\mathscr{0} \\
\stackrel{0}{N}\end{array}$ & 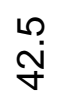 & స్ & $\stackrel{\overbrace{}}{\stackrel{N}{c}}$ & $\begin{array}{l}\text { ब) } \\
\text { న }\end{array}$ & $\stackrel{\circ}{\circ}$ & $\stackrel{\mathscr{0}}{\dot{0}}$ & $\begin{array}{l}\infty \\
\text { 음 }\end{array}$ & $\begin{array}{l}\mathscr{1} \\
\omega^{\circ}\end{array}$ \\
\hline & ऽ & 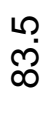 & $\stackrel{\infty}{\infty}$ & $\begin{array}{l}\text { ڤే } \\
\text { مే } \\
\text { Nి }\end{array}$ & $\underset{\dot{\theta}}{\dot{\theta}}$ & $\begin{array}{l}\infty \\
\stackrel{p}{ }\end{array}$ & $\stackrel{\nabla}{\leftarrow}$ & $\underset{\oplus}{+}$ & $\begin{array}{l}\mathscr{\leftrightarrow} \\
\dot{\gamma}\end{array}$ & $\stackrel{\sim}{\stackrel{N}{N}}$ \\
\hline \multirow{3}{*}{$\begin{array}{l}\text { ח̊ } \\
0\end{array}$} & $\overline{0}$ & $\begin{array}{l}\infty \\
\dot{O}\end{array}$ & $\begin{array}{l}\text { @) } \\
\stackrel{N}{ }\end{array}$ & लें & $\stackrel{\widehat{N}}{\stackrel{N}{ }}$ & $\frac{\dot{v}}{m}$ & $\stackrel{\text { m }}{\text { N }}$ & 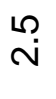 & $\begin{array}{l}\text { g } \\
\text { ठ }\end{array}$ & $\stackrel{m}{\stackrel{m}{\sim}}$ \\
\hline & J & $\begin{array}{l}\mathscr{O} \\
\stackrel{\gamma}{\sigma}\end{array}$ & $\frac{n}{\frac{n}{m}}$ & $\begin{array}{l}\infty \\
\text { هి }\end{array}$ & $\stackrel{\sim}{\sim}$ & $\hat{\stackrel{\omega}{N}}$ & $\stackrel{\circ}{\sim}$ & $\stackrel{\sim}{\sim}$ & $\hat{~}$ & $\stackrel{\text { M }}{\stackrel{N}{\sim}}$ \\
\hline & ర & $\begin{array}{l}\text { में } \\
\text { م্ }\end{array}$ & $\begin{array}{l}\infty \\
\infty \\
\infty \\
\end{array}$ & $\begin{array}{l}\stackrel{\dot{0}}{ } \\
\stackrel{\leftrightarrow}{N}\end{array}$ & $\begin{array}{l}\underset{J}{*} \\
\stackrel{+}{+}\end{array}$ & $\begin{array}{l}\stackrel{\sim}{\sim} \\
\stackrel{\sigma}{\sigma}\end{array}$ & গ̀. & 오 & $\begin{array}{l}\mathscr{0} \\
\stackrel{\rho}{\rho}\end{array}$ & $\begin{array}{l}m \\
\infty \\
\infty\end{array}$ \\
\hline \multirow{3}{*}{ ஸ̃ } & $\overline{0}$ & ?ִ & N & $\begin{array}{l}\text { ڤ̣ } \\
\text { ऽ̊ }\end{array}$ & $\stackrel{\check{\sim}}{\sim}$ & م. & Oٌ & $\begin{array}{l}\infty \\
\infty \\
\infty\end{array}$ & $\begin{array}{l}\infty \\
0\end{array}$ & $\begin{array}{l}\text { న } \\
\text { న }\end{array}$ \\
\hline & త & $\stackrel{\circ}{\stackrel{\gamma}{\gamma}}$ & $\begin{array}{l}\underset{\infty}{\infty} \\
\infty\end{array}$ & \begin{tabular}{l}
$\cong$ \\
\multirow{g}{\Xi}{}
\end{tabular} & $\begin{array}{l}\text { の. } \\
\infty\end{array}$ & ભु & $\bar{\sim}$ & $\begin{array}{l}\text { వి } \\
\infty\end{array}$ & $\stackrel{+}{\circ}$ & $\hat{\infty}$ \\
\hline & ৫ & ঙ্ল & 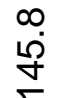 & $\stackrel{\text { N }}{\text { N }}$ & $\bar{\sigma}$ & 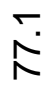 & $\stackrel{\check{\sim}}{\widetilde{\gamma}}$ & ڤு & $\hat{\stackrel{\gamma}{Y}}$ & $\begin{array}{l}\text { क् } \\
\text { ले }\end{array}$ \\
\hline \multirow{3}{*}{$\begin{array}{l}\bar{m} \\
\mathbf{o}\end{array}$} & $\bar{c}$ & مُ & $\stackrel{m}{N}$ & ז' & $\begin{array}{l}\dot{\nabla} \\
\text { ம. }\end{array}$ & $\stackrel{\sim}{\sim}$ & $\stackrel{+}{\sim}$ & $\begin{array}{l}\forall \\
\dot{0}\end{array}$ & $\check{F}$ & 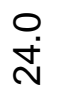 \\
\hline & ช్ & $\begin{array}{l}0 \\
\stackrel{0}{R}\end{array}$ & $\stackrel{\circ}{\stackrel{\infty}{N}}$ & $\begin{array}{l}0 \\
\stackrel{0}{N} \\
\underline{1}\end{array}$ & $\underset{\sim}{\stackrel{\sim}{+}}$ & $\overline{\mathscr{N}}$ & $\stackrel{\sim}{\sim}$ & $\underset{⿱ ⺌}{\forall}$ & $\begin{array}{l}\infty \\
\stackrel{\infty}{0} \\
\frac{1}{c}\end{array}$ & 옹 \\
\hline & ৫ & 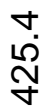 & $\check{\circ}$ & $\hat{N}$ & $\begin{array}{l}\text { ○े } \\
\text { ले }\end{array}$ & $\dot{\varphi}$ & $\begin{array}{l}\text { @) } \\
\text { ¿ }\end{array}$ & $\stackrel{\infty}{N}$ & $\frac{5}{\sigma}$ & $\stackrel{+}{\stackrel{\leftrightarrow}{N}}$ \\
\hline 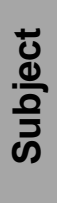 & 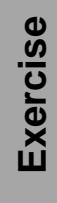 & 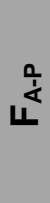 & 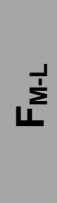 & $\underset{\leftarrow}{\stackrel{x}{\leftarrow}}$ & $\sum_{\Sigma}^{3}$ & $\stackrel{\stackrel{u}{\dot{u}}}{\Sigma}$ & 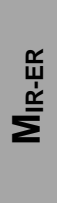 & $\begin{array}{l}\frac{0}{0} \\
\frac{1}{4} \\
⿱ 亠 乂 \\
⿱ 亠 乂\end{array}$ & $\begin{array}{l}\frac{0}{\sigma} \\
\frac{c}{\alpha} \\
\text { 山' } \\
\text { ப' }\end{array}$ & 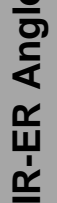 \\
\hline
\end{tabular}




\section{B: DESIGN}

The following sections describe the design process and considerations taken in the development of the pedal system and the modification of the upright static bicycle. This includes the system requirements, the equipment used, the assembly concept, the modifications planned, and the integration of the system with a motion analysis system.

\section{B.1 System Requirements}

The upright static bicycle is intended to be used in biomechanics experiments and thus has to meet certain requirements. These requirements should allow for repeatable, reliable measurements of forces at the feet of the rider, as well as output data in the standard format (against crank angle) to easily compare results with published results. Some requirements include data quality, unmodified biomechanics on the static bicycle compared to normal (unmodified) bicycles, and integration with the Motion Analysis system to be used in cycling biomechanics experiments. Table B.1 lists the requirements defined at the beginning of the project and a planned way to test its fulfillment.

Table B.1 System requirements and testing plan.

\begin{tabular}{|c|l|c|}
\hline Spec & \multicolumn{1}{|c|}{ Parameter Description } & Compliance \\
\hline 1 & Bike must not change rider kinematics & Test \\
\hline 2 & $\begin{array}{l}\text { Wires and added mass (load cells) at pedals must } \\
\text { not change rider feel }\end{array}$ & Test/Analysis \\
\hline 3 & Measure forces and moments in three dimensions & Inspection \\
\hline 4 & Load cell data must not be affected by vibrations & Inspection \\
\hline 5 & Integrate with motion analysis system & Test \\
\hline 6 & Output data in crank angle (standard) & Inspection \\
\hline 7 & $\begin{array}{l}\text { Modified pedal must support expected maximum } \\
\text { loading }\end{array}$ & Analysis \\
\hline
\end{tabular}


Adherence to each of the parameters described above is imperative for the success of the project. More details about each parameter follow:

- Rider feel of the modified bicycle is important to help the riders maintain the cycling position and aid the rider ignore the testing equipment. If the load cells change the foot position with respect to the original position (i.e. no load cells present), kinematics may be changed for the rider, yielding different knee loads. If the rider is conscious of the test equipment, they may alter their biomechanics.

- If the wires attached to the bicycle or the added mass at the pedals by the load cells change the rider experience of the bicycle, the rider may change riding position or riding effort, deviating from normal cycling knee loads.

- The modified pedals must record forces and moments in three orthogonal directions so a three dimensional inverse dynamics problem can be solved looking for resultant knee loading during cycling.

- Vibrations may alter the load cell data, which in turn can propagate through the calculations, giving calculated knee loads that are noisy and/or not usable. Vibrations must be controlled in order to ensure the quality of the data.

- The integration of the modified static bicycle with the motion analysis system already present at the HMB Lab is essential since this is the system used to run biomechanics experiments.

- The output format for the knee load data makes comparing results with published values in the literature possible. These comparisons are needed as a way of validating the results obtained with the system.

- The modified pedal and load cells must be able to support the expected loads. A failure of these parts would stall any study involving the system. A search of published values can help determine an expected range. 
The parameters that have to do with the feel of the modified static bicycle (how riders experience the bicycle) can be validated by testing (asking volunteers to ride the bicycle and provide feedback) and analysis (to see effect of added mass at pedals on rider). Load cell data quality can be inspected by plotting the data obtained from the load cells and checking for three force and moment components as well as smoothness of data (removing noise from data). The integration of the modified bicycle with the motion analysis system can be tested by running an experiment. If an experiment can be performed successfully, the integration with the system is complete. Crank angle output can be done by having a custom code process the data and provide the desired format. Lastly, the ability of the modified pedals to hold expected loading can be checked by preforming stress analysis.

\section{B.2 Pedal Concept}

It was known that load cells had to be used and placed at the petals. A force plate is far too large to be placed in this location. The initial idea was to place the load cells on top of the pedals. This may bring a problem as the distance of the foot with respect to the

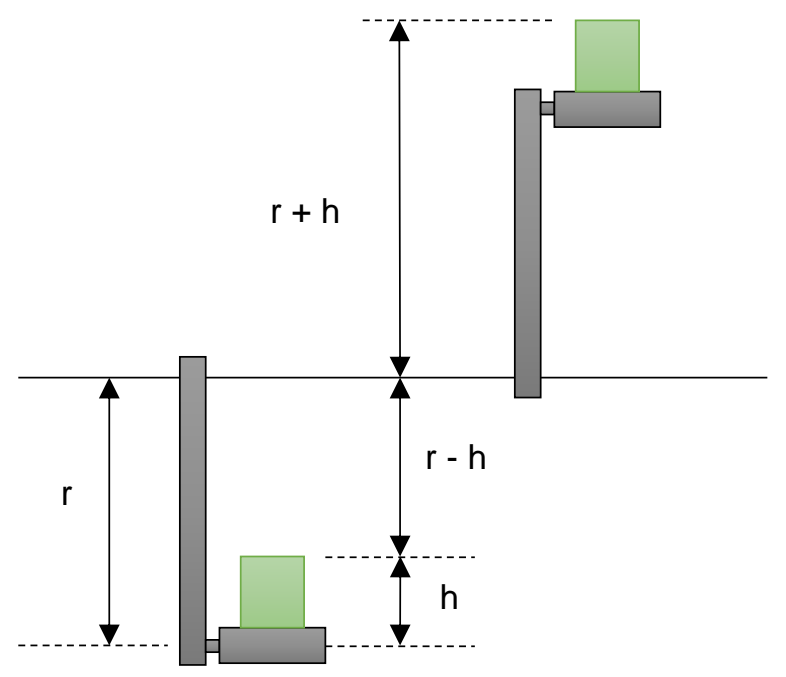

crank center is different when the pedal is at top dead center (TDC) or at bottom dead center (BDC). Figure B.1 describes this. At TDC, the distance from the crank center is larger than when the pedal is at BDC.

Figure B.1 Assembly with load cell on top of pedal. 
To solve this issue the load cell can be lowered into the pedal. This would require that the pedal have no spindle. By locating the load cell so that the foot of the rider is placed at the same location it would be located for a normal, unmodified pedal, the same rider experience

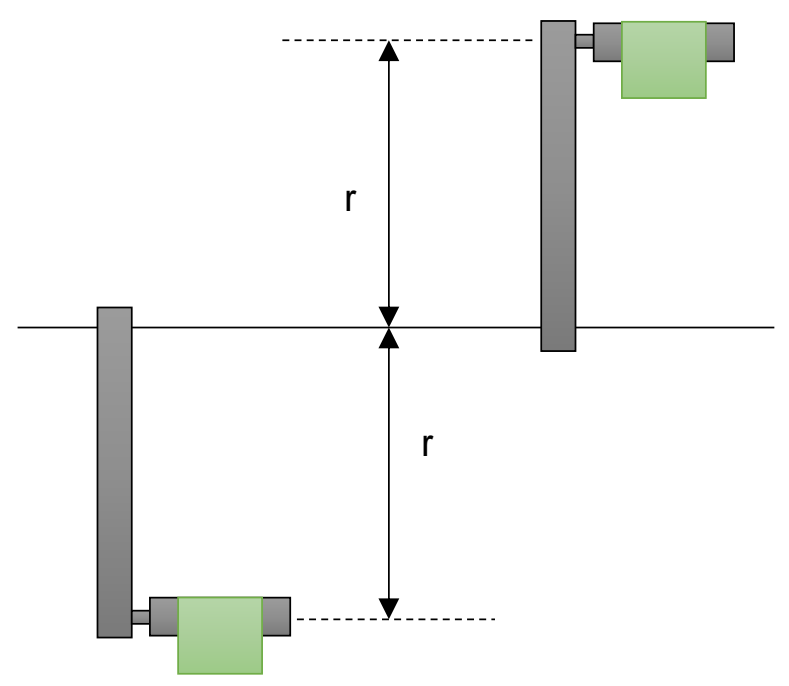

Figure B.2 Proposed load cell position within pedal body.

can be achieved. Figure B.2 shows the geometry with the proposed load cell location. The differences in foot location with respect to the crank center at TDC compared to BDC are greatly reduced. In this figure, the thickness of the pedal is assumed negligible compared to the thickness of the lead cell.

\section{B.3 Equipment}

The equipment selection is described in the following section. Included are the motion analysis system, the load cells, the upright static bicycle, and the pedals. The last three were obtained with the proposed assembly mentioned above in mind. It was important that the static bicycle selected allowed for easy retro-fitting and that the pedals have no spindle and a bearing system that can support the expected loads.

\section{B.3.1 Motion Analysis System}

The HMB Lab has a complete motion analysis system. This system includes the software Cortex, eight near-infrared Owl Cameras (Motion Analysis Corporation, Santa Rosa, CA), AMTI Accugait force plates for gait experiments (AMTI, Watertown, MA), and a NI USB 6218 data acquisition system (National Instruments, Austin, TX). A gait-focused description follows. 
To run biomechanics experiments, subjects have retro-reflective markers attached at specific anatomical landmarks. The markers are located on the body based on the specific marker set used for the experiments. These markers attach to the skin using stickers and Velcro pieces (see figure B.3). The Owl Cameras emit a near-infrared (750 nm wavelength) light using an array of LEDs located around the lens of the camera. This light hits the markers and bounces back into the lens, picking up the position of the markers in the two dimensional field of view of the camera. The cameras are positioned and optimized to pick up markers in the

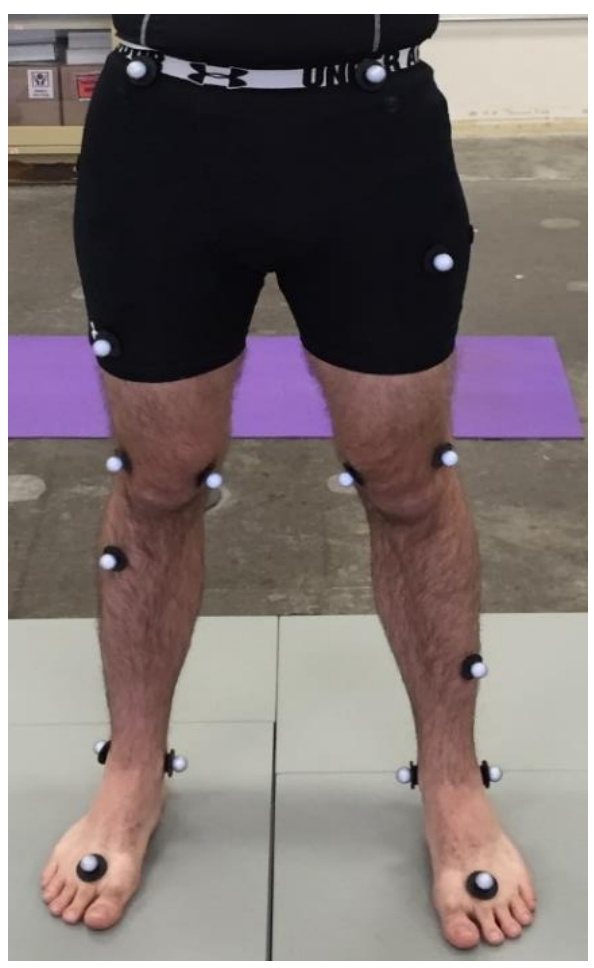

Figure B.3 Retro-reflective marker on subject.

middle of the room, hereby called the capture volume. One camera is defined as the master camera. The master camera outputs the clock signal (square wave with frequency equating frames per second recorded) used for data synchronization.

Cortex is a motion analysis software developed by Motion Analysis Corporation that receives information from the infrared cameras and all the force plates and load cells, synchronizes all kinematic (camera) and kinetic (force plates and load cells) data, and solves the inverse dynamics problem to calculate resultant knee joint loads. This software knows the position and orientation of all the cameras in the system. These settings have been input during the system set up and calibration. The cameras have been represented relative to one another in the software. When a camera picks up a maker, the information is used by Cortex to calculate the position of the marker in the room. At least two cameras must pick up the marker for the system to accurately position it in the global coordinate 
system (virtual representation of the lab space). One camera is able to locate a marker in two dimensions based on its field of view. When two cameras are able to see the same marker, the location of the marker can be found by combining both fields of view. This triangulation requires two cameras to see the marker, but the more cameras see the same marker, the more accurate the positions can be. Once a marker has been located, Cortex stores its position in the global coordinate system using $x, y$, and $z$ directions. Tools in the graphic user interface of the software allow the user to name each marker based on the anatomical position of the marker and expected marker set used. Sometimes a marker can be lost by the system, leaving frames with missing markers or with marker confusion (the system cannot follow the path of the marker correctly). The marker trajectories can be processed using a cubic joint tool (to fill in frames missing position information) and a low pass, two-pass, forth order, zero phase shift Butterworth filter [25] (to smooth marker trajectories and reduce noise). Figure B.4 shows a screen shoot of Cortex in use.

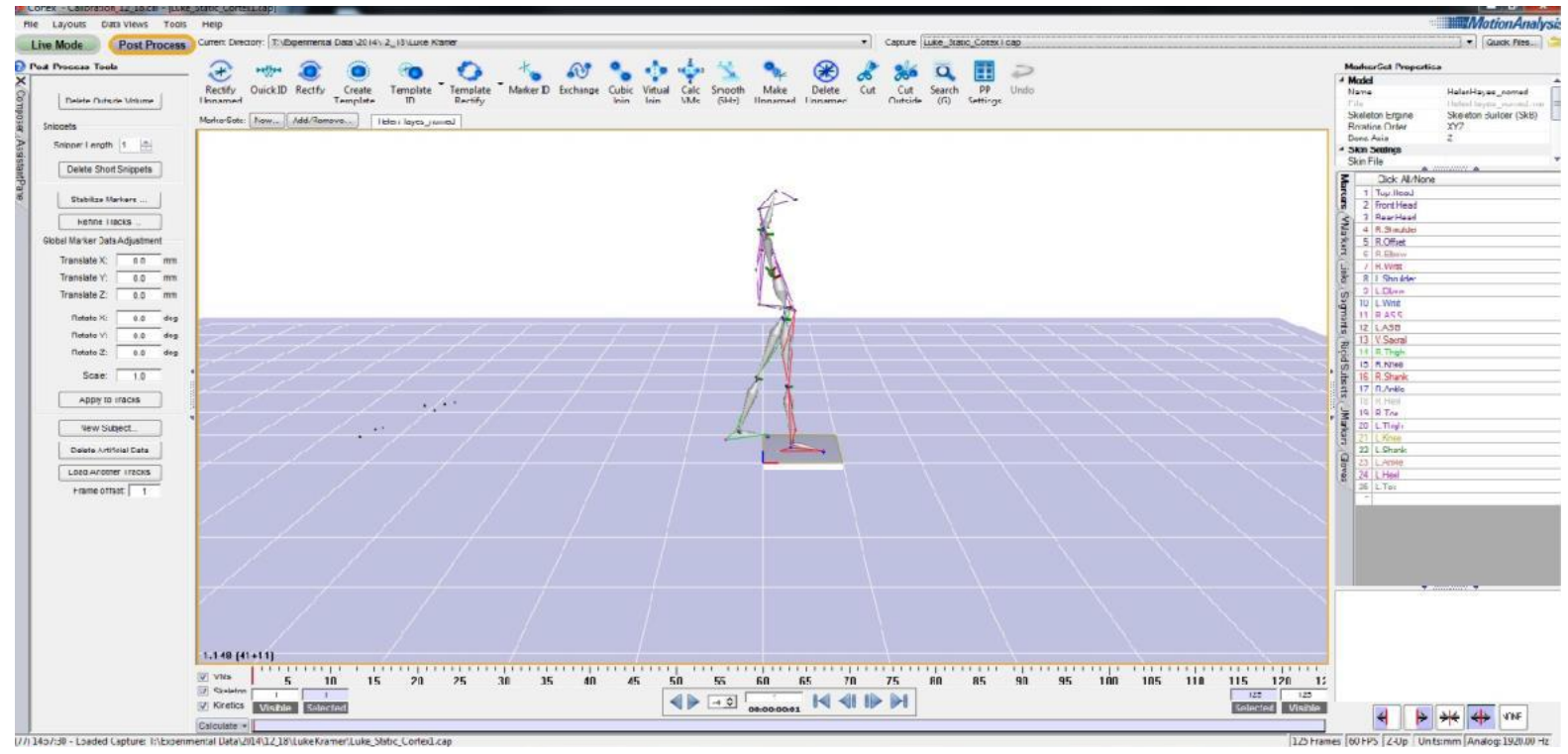

Figure B.4 Cortex graphical interface. Gait trial shown with markers already identified. 
Each marker must be named for the system to recognize it and determine which body part each marker belongs to. This nomenclature is done using a marker set. A marker set is a template for locating retro-reflective markers on subjects at standard anatomical positions. The Helen Hayes $(\mathrm{HH})$ marker set is often used in biomechanics experiments at the HMB Lab. Figure B.5 shows the anatomical locations used in the lower body $\mathrm{HH}$ marker set. Table B.2 shows the name of each marker set based on the numbers in the figure and their rough location. The $\mathrm{HH}$ marker set was selected to use the predefined SkyScript (Cortex code language) files that model the subject based on that marker set. While the $\mathrm{HH}$ marker set is simple to implement and modeling tools are readely available in Cortex, there are some limitations to it. The lateral and medial ankle markers define the axis of rotation for the anke. The heel and toe markers define the direction of the foot. These too markers are insufifient to fully define foot orientiaon, for example rotation of the foot about its axis. Furthermore, the varus/valgus angle at the knee is very small. The error in marker position may be too large to accurately predict this knee angle.
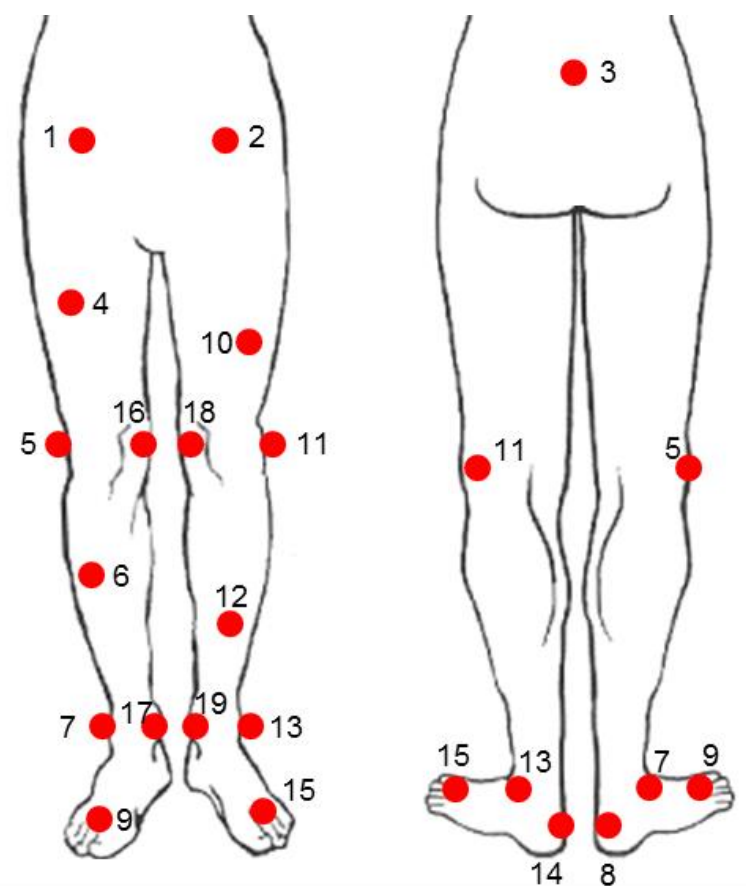

Figure B.5 Helen Hayes marker position. 
Table B.2 Helen Hayes marker set description.

\begin{tabular}{|c|l|l|}
\hline No & \multicolumn{1}{|c|}{ Name } & \multicolumn{1}{c|}{ Anatomical Location } \\
\hline 1 & R.ASIS & Right Anterior Superior lliac Spine \\
\hline 2 & L.ASIS & Left Anterior Superior lliac Spine \\
\hline 3 & V.Sacral & Sacrum \\
\hline 4 & R.Thigh & Right Thigh (Anterior) \\
\hline 5 & R.Knee & Right Lateral Condyle \\
\hline 6 & R.Shank & Right Shank (Anterior) \\
\hline 7 & R.Ankle & Right Lateral Malleolus \\
\hline 8 & R.Heel & Right Calcaneus \\
\hline 9 & R.Toe & Between Second and Third Metatarsal Right Foot \\
\hline 10 & L.Thigh & Left Thigh (Anterior) \\
\hline 11 & L.Knee & Left Lateral Condyle \\
\hline 12 & L.Shank & Left Shank (Anterior) \\
\hline 13 & L.Ankle & Left Lateral Malleolus \\
\hline 14 & L.Heel & Left Calcaneus \\
\hline 15 & L.Toe & Between Second and Third Metatarsal Left Foot \\
\hline 16 & R.Knee.Medial & Right Medial Condyle \\
\hline 17 & R.Ankle.Medial & Right Medial Malleolus \\
\hline 18 & L.Knee.Medial & Left Medial Condyle \\
\hline 19 & L.Ankle.Medial & Left Medial Malleolus \\
\hline
\end{tabular}

The process to use Cortex is quickly described next:

- Calibraiton: Tells the system where all the cameras and force plates are in the room and allows the system to determine the error of the marker positoins.

- Marker Set Identification: To record data, a marker set must be decided on. This marker set will define the modeling possible in Cortex.

- Marker Set Placement: Retro-reflective markers are placed on the subject at the anatomical landmarks corresponding to the marker set.

- Data Adquisition: Run the experiment and have Cortex record the data.

- Marker Identification: Use the graphical user interface (GUI) to name each marker in a frame. 
- Marker Processing: "Rectify" (extending marker identification across all frames), "Cubic Join" (filling gaps in marker trajectory), and "Smooth" (filtering) tools are used in the GUI of Cortex.

- Modeling: Use of the SkyScripts to model the marker set. This creates a virtual representation of the body segments in the virtual representation of the lab. This code also defines the joint centers and the axis of rotation of each segement.

Two AMTI Accugait force plates are placed in the capture volume (figure B.6). These force plates are used for gait experiments. Subjects must walk across both force plates which are staggered to facilitate subject gait without targeting (purposely changing gait to hit the force plates). The loads applied on the force plates are recorded and used by Cortex to find solve the inverse dynamics problem (calculate knee joint loads). Each force plate has eight analog output channels that connect to the NI USB 6218 Analog to Digital Converter (ADC). The ADC also has a clock signal that comes from the master camera. The ADC uses the clock signal to time stamp the analog force data from the force plates. The data is then sent to the computer via USB for use in Cortex.

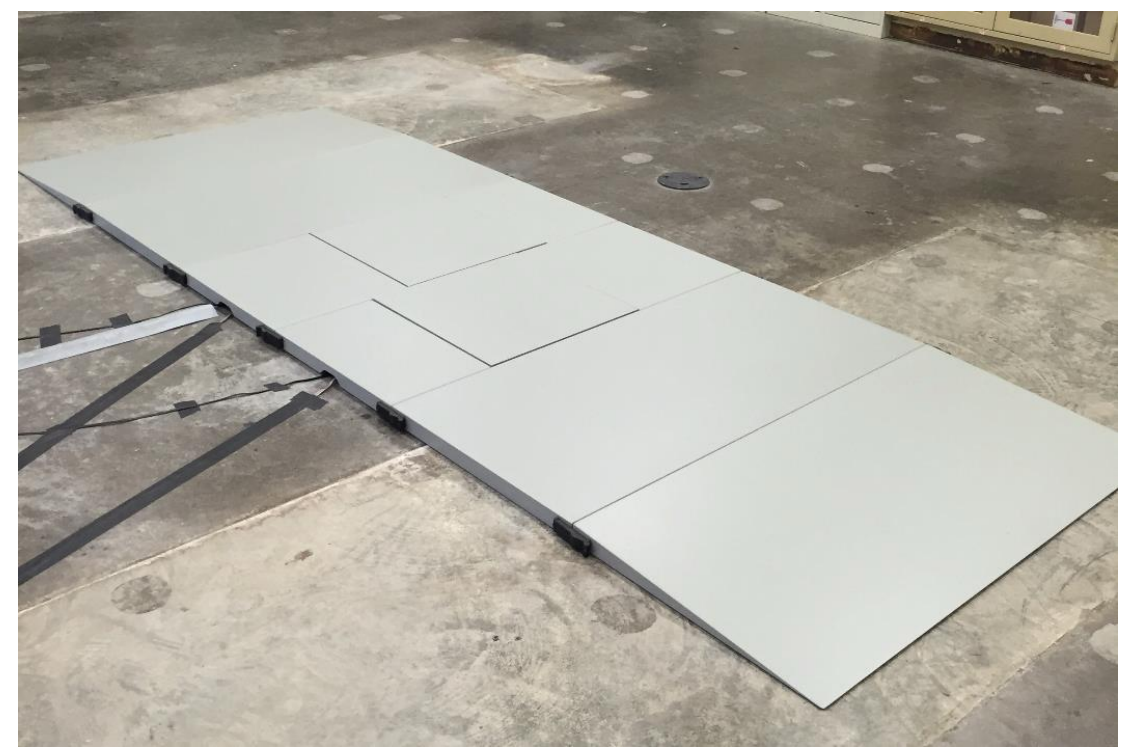

Figure B.6 AMTI Force Plates. 
Once data has been recorded on Cortex, the markers have been identified, and the marker paths have been cleaned up (filled empty frames and filtered), the system can calculate forces and moments at joints (done by a SkyScript code). Cortex outputs these loads for the ankle, knee, and hip joint in gait experiments using the lower body $\mathrm{HH}$ marker set. To find these loads, Cortex uses the location of each marker to calculate the position and orientation, velocity, and acceleration of each body segment (foot, shank, thigh, and pelvis). This kinematic data is combined with kinetic data (from the force plates in the case of gait experiments) and synchronized using the time stamp from the clock signal. Basic dynamics problems are based on the sum of forces (Eq B.1) and sum of moments (Eq B.2) to solve a system of equations for unknown values.

$$
\begin{gathered}
m \vec{a}=\Sigma \vec{F} \\
I \vec{\alpha}+\vec{\omega} \times I \vec{\omega}=\Sigma \vec{M}
\end{gathered}
$$

where $m$ and $a$ are the mass and acceleration of the body segment, $F$ are forces summed, $I, \omega, \vec{\alpha}$ the moment of inertia, angular velocity and angular acceleration of each body segment respectively, and $M$ are moments summed. Using equations 1 and 2 in three dimensions yields a set of six equations used to find loads at a joint (Eq B.3 - B.8) [26].

$$
\begin{array}{cc}
\Sigma F_{X}: R_{X p}-R_{X d}=m a_{X} & \text { Eq B.3 } \\
\Sigma F_{Y}: R_{Y p}-R_{Y d}-m g=m a_{Y} & \text { Eq B.4 } \\
\Sigma F_{Z}: R_{Z p}-R_{Z d}=m a_{Z} & \text { Eq B.5 } \\
\Sigma M_{X}: I_{X} \alpha_{X}+\left(I_{Z}-I_{Z}\right) \omega_{Z} \omega_{Y}=R_{X d} L_{d}+R_{X p} L_{p}+M_{X p}-M_{X d} & \text { Eq B.6 } \\
\Sigma M_{Y}: I_{Y} \alpha_{Y}+\left(I_{X}-I_{Z}\right) \omega_{X} \omega_{Z}=R_{Y d} L_{d}+R_{Y p} L_{p}+M_{Y p}-M_{Y d} & \text { Eq B.7 } \\
\Sigma M_{Z}: I_{Z} \alpha_{Z}+\left(I_{Y}-I_{X}\right) \omega_{Y} \omega_{X}=R_{Z d} L_{d}+R_{Z p} L_{p}+M_{Z p}-M_{Z d} & \text { Eq B.8 }
\end{array}
$$


where $R_{X p}, R_{Y p}, R_{Z p}$ are the proximal joint reaction forces, $R_{X d}, R_{Y d}, R_{Z d}$ are the distal joint reaction forces, $m$ is the segment mass, $a_{X}, a_{Y}, a_{Z}$ are the linear accelerations of the segment center of mass, $g$ is the acceleration due to gravity (Cortex uses $9.8 \mathrm{~m} / \mathrm{s}^{2}$ ), $L_{d}$ and $L_{p}$ are the distal and proximal distances from the center of mass to the distal and proximal joints, respectively, $I_{X}, I_{Y}, I_{Z}$ are the components of the moment of inertia, $\alpha_{X}$, $\alpha_{Y}, \alpha_{Z}$ are the components of angular acceleration, $\omega_{X}, \omega_{Y}, \omega_{Z}$ are the components of angular velocity, $M_{X d}, M_{Y d}, M_{Z d}$ are the distal joint moments, $M_{X p}, M_{Y p}, M_{Z p}$ are the proximal joint moments. Subscripts $X, Y$ and $Z$ refer to the orthogonal directions where $Y$ is the vertical direction. The typical unknowns are $R_{X p}, R_{Y p}, R_{Z p}, M_{X p}, M_{Y p}$, and $M_{Z p}$ (proximal reaction forces and moments). The reaction forces are found first. [26]

Data from the force plates or loads cells, if applied at the feet (like in gait or cycling), is referred to as the Ground Reaction Force (GRF). The kinematic information of the foot is combined with the GRF data to solve loads at the ankle joint (proximal terms for foot equations). These loads are inverted and applied to the distal end of the shank. The calculations are repeated solving for knee loads (proximal end of the shank). The code continuous this process until the top of the model is reached and no more segments are left to calculate. When the lower body $\mathrm{HH}$ marker set is used, the segments (virtual representations of body parts) present are the feet, shanks, thighs, and the pelvis. Other useful information outputted by Cortex is joint angles for the joints in the model. Measurements of joint angles during motion show differences in motion and biomechanics amongst subjects.

Once the solver has calculated all joint forces, moments, and angles for the model, the data is displayed in presentation graphs. Data in presentation graphs is shown in anatomical directions and not in the global coordinate system. The data in presentation 
graphs can be saved in ".data” files. All maker positions in global coordinate system are saved in ".trc" files. Both file extensions can be processed in Excel.

\section{B.3.2 Load Cells}

Based on the pedal assembly concept mentioned above, the load cells needed must be small enough to fit inside a bicycle pedal or have a size approximate to a pedal. The loads cells must record force and moment data in three dimensions and be recognizable by Cortex. Furthermore, the data from the load cells should be amplified and conditioned to remove noise. The load cells chosen were the AMTI AD2.5D250, which are used with the GEN 5 signal conditioners (figure B.7)
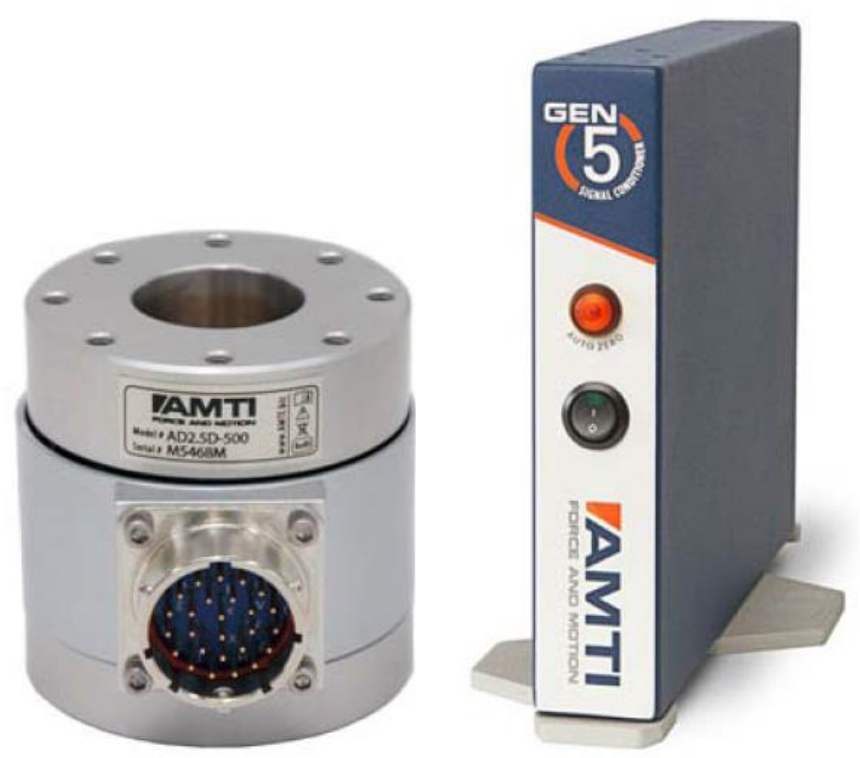

Figure B.7 Load cell and signal conditioner selected. Images from amti.biz.

The AMTI AD2.5D-250 dimensions are 2.5 inches tall by 2.5 inches in diameter. The load cell uses a strain gage bridge as its sensing elements. The crosstalk is less than $2 \%$ on all channels. The maximum physical capacities for each channel are described in table B.3. Each channel is independently configurable. The software AMTI NetForce can be used to adjust load cell excitation, gain, and DC set point for each channel. If a measurement for a specific channel is expected to be much smaller than the physical capacity, the range of measurement should be adjusted to improve resolution by changing the gain and excitation settings to set the electronic capacity. In case the measurement exceeds the electronic capacity, no damage is done to the load cells but the data is not 
reliable and should not be used. If the measurements barely exceed the capacity of the load cell, damage may not occur but data should not be used. Exceeding physical capacity of the load cell risks permanent damage to the load cells. Care must be taken to make sure both the electronic and the physical capacity of the load cells are not exceeded. For better results, a one hour warm-up period should happen before testing.

Table B.3 AMTI AD2.5D-250 load cell maximum physical capacity.

\begin{tabular}{|c|c|c|c|c|c|}
\hline \multirow{2}{*}{ Channel } & \multicolumn{2}{|c|}{ Capacity } & \multirow{2}{*}{ Channel } & \multicolumn{2}{|c|}{ Capacity } \\
\hline & [lbs.] & [N] & & [lbs-in] & {$\left[\mathrm{N}^{*} \mathrm{~m}\right]$} \\
\hline$F_{x}$ & 125 & 556 & $M_{x}$ & 250 & 28 \\
\hline$F_{y}$ & 125 & 556 & $\mathrm{M}_{\mathrm{y}}$ & 250 & 28 \\
\hline$F_{z}$ & 250 & 1112 & $\mathrm{M}_{\mathrm{z}}$ & 125 & 14 \\
\hline
\end{tabular}

The GEN 5 Signal Conditioner uses the calibration matrix provided by AMTI to turn voltage from the load cells into usable force and moment measurements. Multiple types of signal conditioning are implemented including a $1 \mathrm{kHz}$ anti-aliasing filter, oversampling and digital signal processing. The GEN 5 performs numerical processing including the use of factory calibrated constants in place of nominal values for gains and excitations, correcting for cable losses due to finite bridge resistances, and providing crosstalk corrections. These filtering options allow for clean data to be reliably and repeatedly recorded.

\section{B.3.3 Static Bicycle}

The Life Fitness Lifecycle GX (seen in figure B.8) was chosen from the options discussed below. This bike was chosen for its ease to retrofit, ground clearance, resemblance to true bicycle, weight properties, easy of mobility, and repeatability in resistance level selection. Some of the bikes considered had bulky plastic coverings that could get on the way when the crank of the bicycle is modified. This ties in with the ground 
clearance. If the pedal body is extended under the pedal surface, the pedal may hit the ground; therefore, sufficient ground clearance is required. The resemblance of this bike with real bicycles is beneficial as measurements of the loads on the knee during biking is needed. Similarity between this upright static bicycle and a real bicycle will give the rider the same experience as if riding a normal bicycle and produce more accurate results. Next, a bicycle that is heavy and is able to be used with obese individuals is needed. The heavier mass of the bike will make it move less while in use which decreases the noise in the reading of the forces at the pedal and facilitate vibration isolation. Obese subjects may be used in some experiments, therefore strength of the frame is

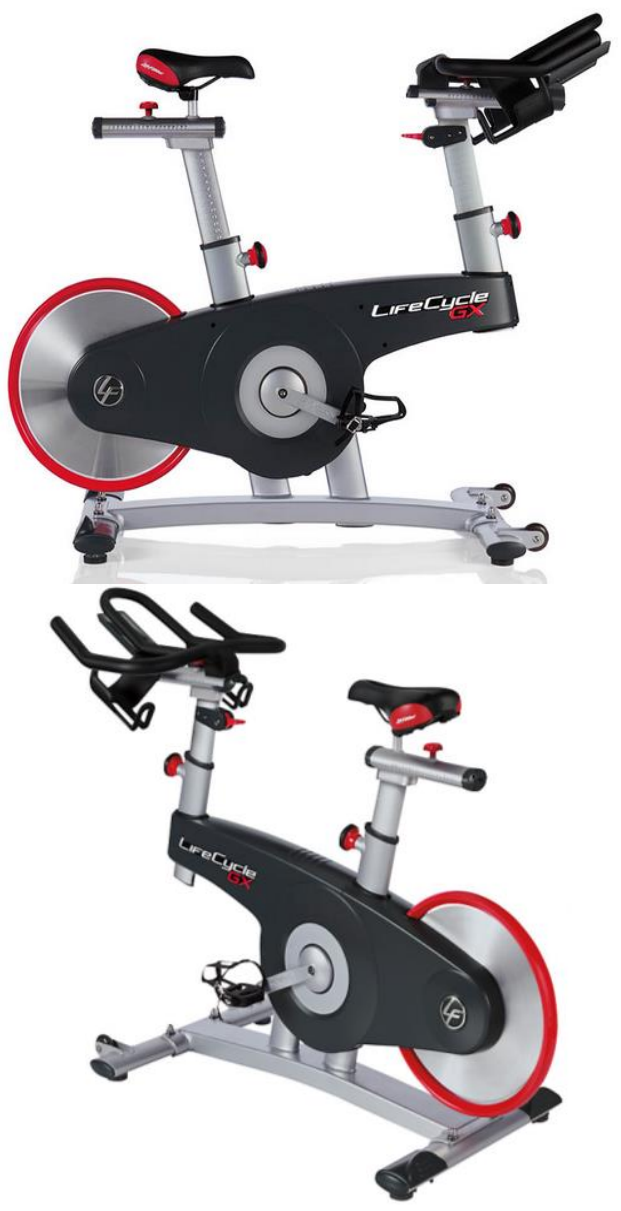

Figure B.8 Life Fitness Lifecycle GX. Upright static bicycle selected. Images from lifefitness.com. required in order to be able to use the bike for obese biomechanics experiments. The lab is used for many types of experiments; whatever experiment is being performed must be carried out in the capture volume. Due to this, the bike must be easily moveable in the lab. Despite its weight, the Lifecycle GX can be effortlessly moved by holding the handle bars and letting the bicycle roll on the two front wheels. Lastly, the Lifecycle GX has 20 resistance levels with a digital readout allowing for quick reselection of a specific resistance level. The resistance system in this bicycle is a dual magnetic brake adjusted by a resistance braking lever. This device uses the spinning flywheel in the back of the bicycle and magnets to create eddy currents that oppose the motion of the flywheel, thus creating a resistance for the rider. By accurately reproducing the same resistance level for 
all subjects, intensity of cycling can be controlled. The Life Fitness Lifecycle GX had the best combination of higher mass and maximum user weight, less housing flaring out that could interfere pedal modifications, better ground clearance, best resemblance to normal bicycles, and ease of mobility and repeatability of resistance level. The following discussion briefly compares this bicycle to the other options considered before selection.

Since a gym quality upright static bicycle is needed, the Recreation Center at Cal Poly served as a first place to find brands of good quality. Three upright static bicycles were found. Although the same models could not be found with vendors, other options by the same manufacturers were explored. From Schwinn, the best option based on requirements mentioned was the IC2 Indoor bike (see figure B.9 A). This bicycle weights $83 \mathrm{lbs}$. and has a maximum rider weight of $250 \mathrm{lbs}$. The resistance levels did not seem repeatable due to the advertised "infinite levels of resistance". The cost at the time of search was $\$ 599$. Next, bicycles by Precor were explored. The UBK 615 Upright bike (see figure B.9 B) weighted $155 \mathrm{lbs}$. but a subject up to $350 \mathrm{lbs}$. could use it. It offers 25 discrete levels of resistance. The price found at the time was $\$ 2345$. In this model, the housing seemed too bulky which could be an issue when retro-fitting the pedals and the price was too high. Last, Life Fitness bicycles were searched. Three models seemed acceptable. The C1 Lifecycle (figure B.9 D) and C3 Lifecycle (figure B.9 E) are very similar with the C3 being heavier (118 lbs.) and allowing subjects up to $400 \mathrm{lbs}$. compared to the $300 \mathrm{lbs}$. maximum user weight for the C1 Lifecycle (105 lbs.). The Lifecycle GX weights 111 lbs. and allows users up to $350 \mathrm{lbs}$. The three models have 20 resistance levels, however, the Lifecycle GX costs $\$ 1799$, with the C1 and C3 Lifecycle at $\$ 1399$ and $\$ 1899$ respectively. Table B.4 summarizes these information. The Lifecycle GX was chosen because of its balance in cost, weight, resistance levels, and user weight capacity. 


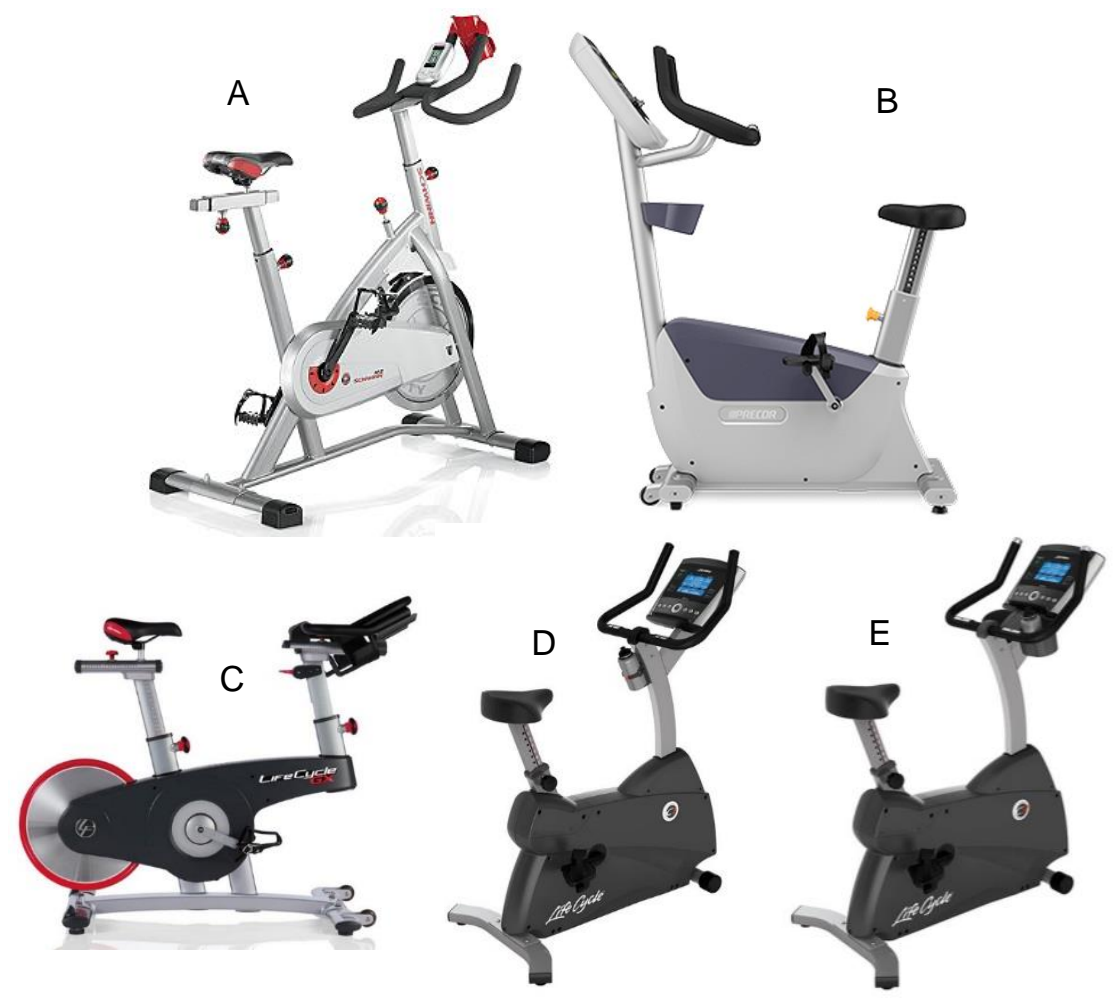

Figure B.9 Bicycles considered for project. A.) Schwinn IC2. B.) Precor UBK 615. C.) Life Fitness Lifecycle GX. D.) Life Fitness C1 Lifecycle. E.) Life Fitness C3 Lifecycle. Images obtained from website of each manufacturer.

Table B.4 Description of static bicycles considered.

\begin{tabular}{|c|c|c|c|c|}
\hline Bicycle & Weight & $\begin{array}{c}\text { User Max } \\
\text { Weight }\end{array}$ & Resistance Levels & Price \\
\hline Schwinn IC2 & $\begin{array}{r}83 \mathrm{lbs} . \\
{[38 \mathrm{~kg}]}\end{array}$ & $\begin{array}{c}250 \mathrm{lbs} . \\
{[113 \mathrm{~kg}]}\end{array}$ & "Infinite levels" & $\$ 599$ \\
\hline Precor UBK 615 & $\begin{array}{r}155 \mathrm{lbs} . \\
{[70 \mathrm{~kg}]}\end{array}$ & $\begin{array}{c}350 \mathrm{lbs} . \\
{[159 \mathrm{~kg}]}\end{array}$ & 25 levels & $\$ 2345$ \\
\hline Life Fitness Lifecycle GX & $\begin{array}{r}111 \mathrm{lbs} . \\
{[50 \mathrm{~kg}]}\end{array}$ & $\begin{array}{c}350 \mathrm{lbs} . \\
{[159 \mathrm{~kg}]}\end{array}$ & 20 levels & $\$ 1799$ \\
\hline Life Fitness C1 Lifecycle & $\begin{array}{r}105 \mathrm{lbs} . \\
{[47 \mathrm{~kg}]}\end{array}$ & $\begin{array}{c}300 \mathrm{lbs} . \\
{[135 \mathrm{~kg}]}\end{array}$ & 20 levels & $\$ 1399$ \\
\hline Life Fitness C3 Lifecycle & $\begin{array}{r}118 \mathrm{lbs} . \\
{[53 \mathrm{~kg}]}\end{array}$ & $\begin{array}{c}400 \mathrm{lbs} . \\
{[180 \mathrm{~kg}]}\end{array}$ & 20 levels & $\$ 1899$ \\
\hline
\end{tabular}




\section{B.3.4 Pedals}

The pedals that came with the Life Fitness Lifecycle GX upright static bicycle have a spindle running through the body of the pedal. In order to locate the load cell as proposed in the pedal concept, the spindle must be removed. Furthermore, the body of the load cell must pass through the body of the pedal without interference. The original pedals have a plastic body therefore removing the spindle will render the pedal too weak to support the expected loads. Tioga produces pedals without spindles with appropriately sized bearings to support these loads. The pedals obtained are the Tioga MT-ZERO (designed for mountain biking) with the Tioga ZERO-Axle bearings (figure B.10). With a normal pedal set up, the spindle supports the loads the rider inflicts on the pedal as well as the bending moment created near the bearing. If the spindle is removed the body of the pedal must support the bending moment. The Tioga pedals come with no spindle from factory and a sized bearing greatly reducing the amount of design, machining, and validation needed as they are expected to support these loads. The bearings come with standard threads which means they will fit the majority of bicycles. If in the future this design needs to be placed on a different bicycle, the bearings will allow for seamless transition.

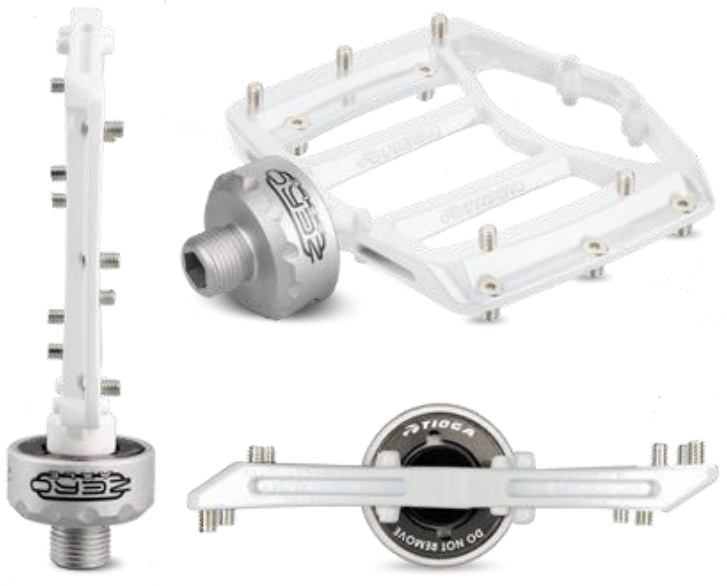

Figure B.10 Tioga MT-ZERO pedals with Tioga ZEROaxle bearings. Image from .tiogausa.com. 
Since the whole pedal body is made from chromoly (steel 4130), the pedal can easily be machined to fit the load cell body. The middle of the pedal body must be removed to allow the load cell to pass through; chromoly will not be damaged by machining. On a final note, the traction pins that come with the pedal can be removed and those holes can be used to attach any element created to house or attach the load cells to the pedals. The low profile of the pedals $(7 \mathrm{~mm}$ thick) allow for the load cell to be placed at the location of the top surface of the original pedal (24 $\mathrm{mm}$ thick) without the feet of the rider touching any other part of the bike which would cause a lower force or moment reading to be measured.

\section{B.4 Load Cell Housing}

A housing is needed to attach the load cells to the pedal, to protect the load cells, and to locate the load cells so that the foot of the rider remains at the same location it would be if the original pedals were in place. The design of these housing is described next.

\section{B.4.1 Requirements}

The pedal concept requires the load cell to pass through the pedal body. A new element must be created to allow for the load cell to stay in position and attached to the pedal. This custom part was designed from scratch to:

- Securely attach the load cells to the pedals: The load cells will be experiencing loads of up to $250 \mathrm{lbs}$. If the load cell is not properly attached to the bike it may fall off causing load cell damage, system failure and all subsequent research to stop until fixed.

- Preserve cycling biomechanics: The new load cell housing must locate the foot of the subject at the same location relative to the spindle axis as the original pedal did. In this way, rider position, feel, and biomechanics of cycling are conserved. 
- Protect load cells: As the crank spins, the load cells will be exposed to being hit by any object that may come in its path. It should also protect the load cells when the bike is not being used or is stored. People in the lab or other objects may hit the pedal. The housing should absorb the hit instead of the load cell body.

- Add rigidity to the pedal body: The middle support bars in the Tioga pedals must be removed to let the load cell pass through. This weakens the pedal body as less material is present to resist bending and shear. The load cell housing can be used to increase the rigidity of the pedal.

- Cable orientation: The load cells must be connected through wires to the GEN 5 signal conditioners. These wires must come outwards to reduce the inertial effects of the wires on the pedals. The load cell housing should orient the load cell so that this requirement is met.

- Do not interfere with measurements: The load cells must measure the true force at the pedals. To do this, the load cell top surface and foot of the rider must not touch anything else other than each other. If the load cell housing touches the load cell anywhere except on the bottom surface (where it should attach), the measured pedal loads cannot be trusted. 


\section{B.4.2 Initial Concept}

The attachment of the load cell to the pedal requires a platform for the load cell to sit on. The load cell can only be attached from the top or bottom surfaces. The top of the load cell is used for measuring the foot forces thus the bottom

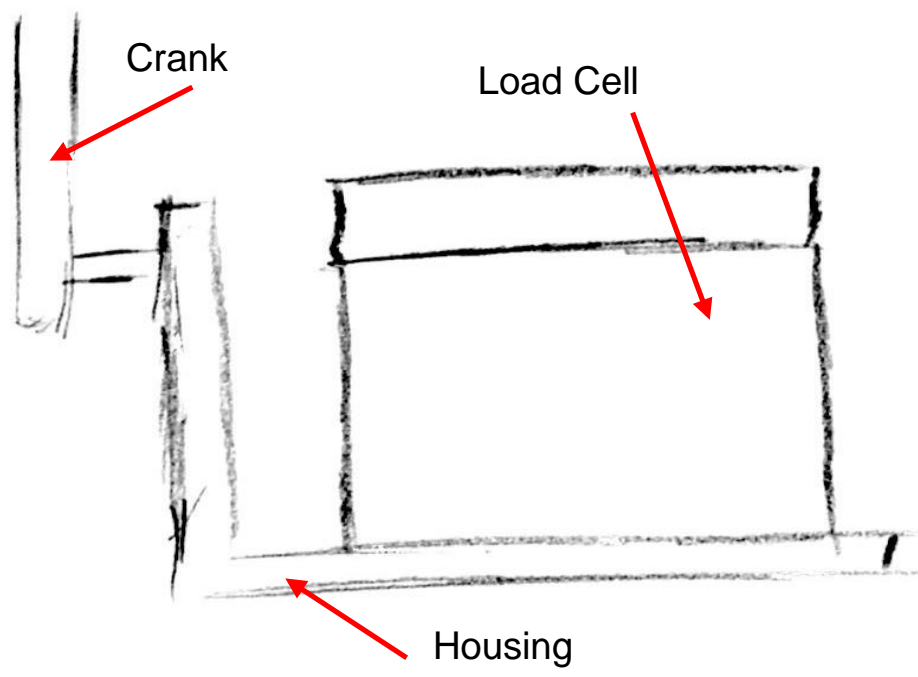
surface must be used for

Figure B.11 Initial idea for load cell housing. attachment. In an effort to reduce mass at the pedal, an L-shape platform was proposed (figure B.11). This would attach to the pedal at the closest part to the bearing. However, the bottom part of the housing where the load cell sits would essentially be a cantilever beam which may deflect too much under load.

To give rigidity to the load cell housing and the weakened pedal body, side walls were added to the side of the housing. The new walls would be able to better resist bending deformation and provide protection to the load cell body. This walls can be used to attach the load cell to the pedal. The final shape is shown in figure B.12. Detail dimensioning follows but first

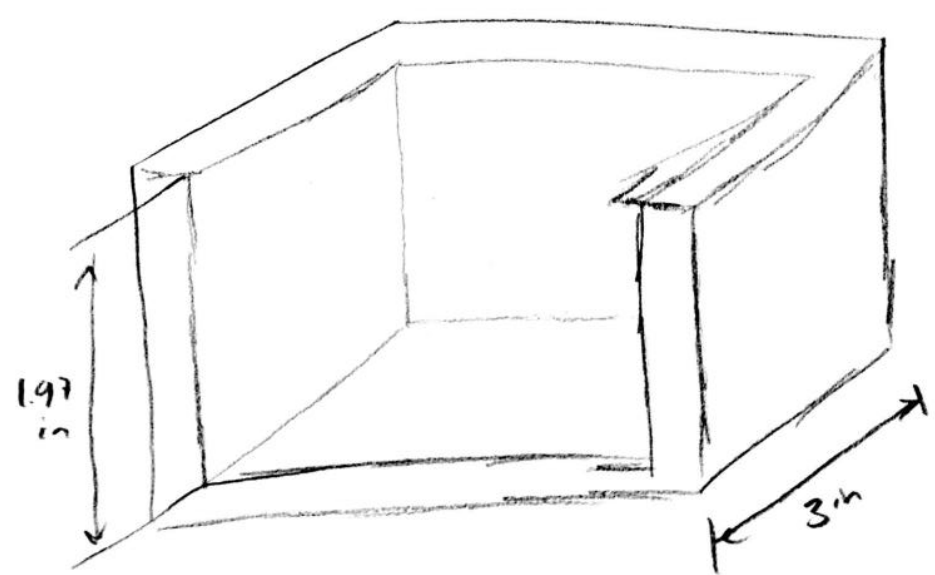
the pedal hole pattern must be known.

Figure B.12 Final shape for load cell housing. No details are defined at this point. 


\subsubsection{Pedal Hole Pattern Dimensioning}

To know where to locate the holes to attach the pedal to the load cell housing, the pedal dimensions must be known. While the general shape of the pedal is not critical, the position of the four holes used for attachment must be located accurately to ensure fit. Attempts to get this information from the manufacturer failed. To get past this issue, the pedal was taken into the Cal Poly shops. With the use of a milling machine and a mechanical

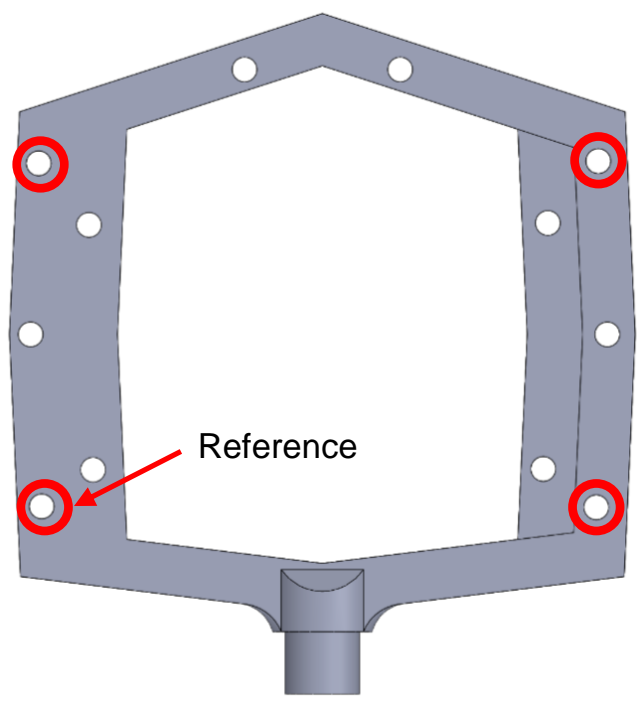

Figure B.13 Model of pedal. Holes to accurately position in red. Bottom left hole is used as reference point for dimensioning.

edge finder with a conical tip, all the holes in the pedal were located with respect to the bottom left hole shown in figure B.13. Only the four holes highlighted where accurately positioned. The other holes were quickly approximated for completeness of the SolidWorks model. The positions of each whole are shown in figure B.14.

$(-0.019,2.172)$

$(3.520,2.186)$

$(0.299,1.782)$

$(3.201,1.794)$

$(-0.070,1.089)$

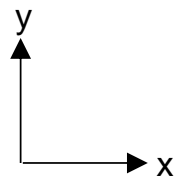

$(3.577,1.089)$

$(0.325,0.232)$

$(3.172,0.237)$

$(0,0)$

(3.507, -0.006)

Figure B.14 Pedal hole pattern dimensions. All dimensions in inches. 


\section{B.4.3 Load Cell Housing Geometry}

Figure B.15 shows the CAD model of the load cell housing. In order to fit and center the load cell into the housing, the cavity was dimensioned to have a width at least 2.5 inches and a depth of 2.75 inches. The round corners are there as it is planned to mill the cavity with a 0.75 inch end mill. To position the foot of the rider as the original pedal would, the cavity is 1.9

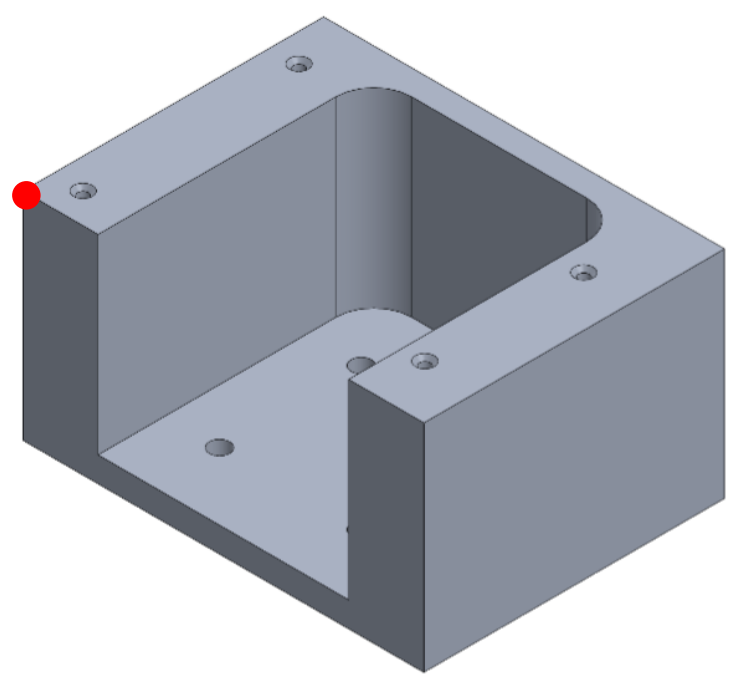

Figure B.15 Load cell housing model. inches deep. This makes the bottom surface of the housing 0.25 inches. The walls end up being 0.75 inches thick. This thickness allows for the four pedal holes to have sufficient material around them. The pedal holes were placed by locating the reference hole 0.300 inches in both directions from the corner signaled with a red circle in figure B.15. The diameter of this holes is done to fit 6-32 UNC screws. The depth was selected to 0.75 inches. Last, the holes to hold and orient the load cells were placed to center the load cell on the cavity and the housing. The clearance holes have a diameter of 0.203 inches (13/64 in). These holes are located from the center of the cavity, radially. This centers the load cell in the cavity, while, the location of the holes aligns the load cell with the housing. It is known that the load cell 8 hole pattern has a 2 inch diameter. In order to drill further away from the housing wall, the holes selected for attaching are the ones at a 45 degree angle. This makes a square that is oriented in the same way as the cavity. Choosing the other four holes creates a diamond shape whose corners run close to all walls and the edge of the cavity. Four $6-32 \times 0.75$ inch countersunk screws are used for attaching each pedal to the load cell housing. Eight 10-32 UNF screws hold each load cell to the pedal platform (4 screws) and the housing (4 screws). 


\section{B.5 Pedal Platform}

Most static bicycles come with straps for the feet of the rider. In order to simulate this type of cycling, straps must be placed on top of the load cell. A pedal platform (figure B.16) was developed to put on top of the load cell surface and add straps to hold the foot of the rider. This platform is easily removable in case other type of seated cycling is required. The pedal platform must only be in contact with the top portion of the load cell and the foot of the rider. Any other contact is not desired as it may introduce loads that are
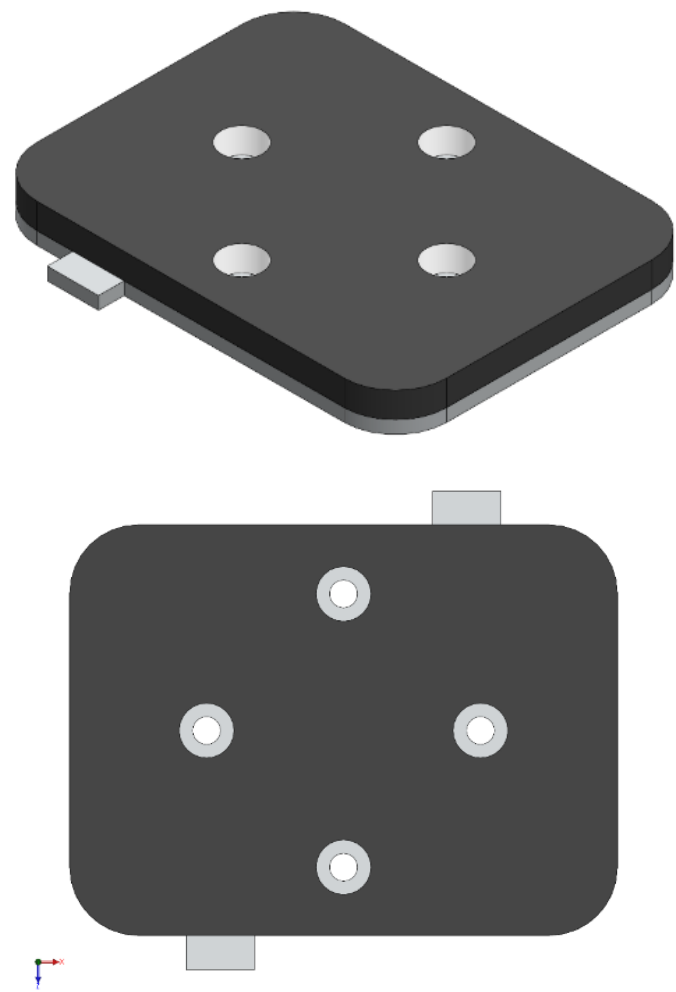

Figure B.16 Pedal platform. not present during seated, upright, strapped cycling.

The material selected for the platform was laminated oil-resistant Buna- $\mathrm{N}$ rubber. This is an aluminum plate with a layer of nitrile rubber (Buna-N). The aluminum side provides structural integrity while the rubber side provides grip for the rider as well as a comfortable surface for the feet. The thickness of the aluminum and rubber parts are 1/8 and 1/4 of an inch, respectively. The tabs on the sides of the platform are used to attach the pedal straps. The platform attaches to the load cell using four screws (10-32 UNF).

The platform is dimensioned to be similar in area to a pedal but not run into the crank or bearing. The tabs on either side are offset to position the pedal straps around the foot of the rider. Regular straps that fit any bike were selected. Each tab has beads of aluminum welded at the edges to hold the straps in place. The rubber was counterbored to hide the screw heads, aiding rider comfort. The corners are rounded for rider safety. 


\section{B.6 System Assembly}

The exploded view of the system assembly is shown in figure B.17. The assembled system is shown in figure B.18. Each pedal has a different assembly as the load cell housing and the pedal platform have slight modifications to account for difference in pedal hole positions (housing) and to improve comfort of pedal strap for the rider. Fasteners are not shown in assembly exploded view. The load cell sits in the housing and is attached with four screws that come up from the bottom (1032). The pedal is then placed on top of the housing. Four screws (6-32) come from the top to hold the pedal and the housing together. The bearing attaches to the pedal using a $4 \mathrm{~mm}$ Ellen wrench as set by the manufacturer. The pedal platform is placed on top of the load cell and secured using four screws (10-32) that come from the top of the platform. The pedal strap completes the assembly. These get pushed through the welded beads to secure the strap in place. To place the assembly on the crank of the static bicycle, an $8 \mathrm{~mm}$ Ellen wrench is used.
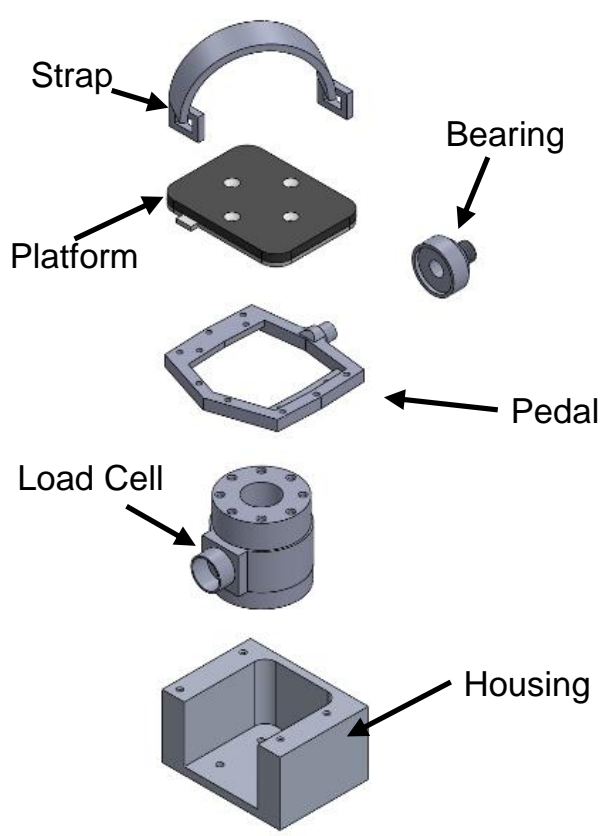

Figure B.17 Pedal assembly exploded view.

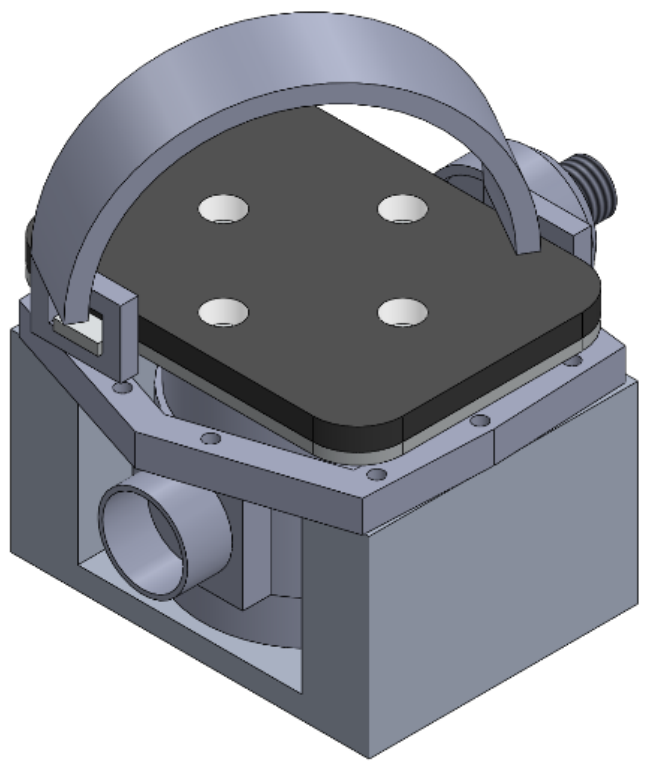

Figure B.18 Pedal assembly collapsed view. 


\section{B.7 Integration with Motion Analysis System}

\section{B.7.1 Gait Set Up}

The HMB Lab set up is shown in figure B.19. There are two Owl cameras on each wall of the lab. The camera left of the HMB computer is designated as the master camera. This camera outputs the clock signal used to synchronize all data. Two AMTI Accugait force plates are placed in the middle of the room (capture volume) and connect to the $\mathrm{NI}$ USB 6218 ADC. The ADC send the force data with a time stamp to the lab computer for Cortex to use.

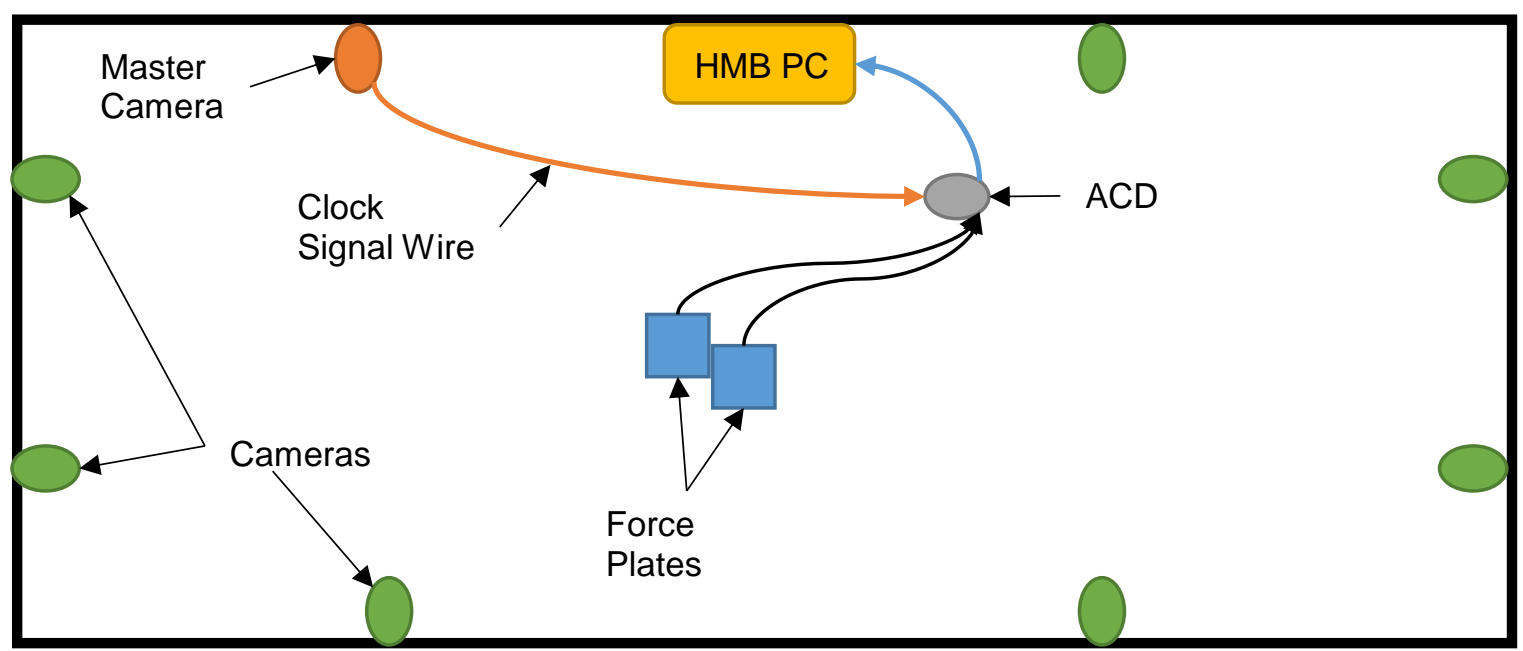

Figure B.19 Layout of HMB Lab.

\section{B.7.2 Cycling Set Up}

Figure B.20 shows the connections of all elements needed for a cycling biomechanics experiment based on the documentation provided by Motion Analysis and AMTI. The static bicycle with the modified pedals is placed in the capture volume (over the force plates). The load cell data wire connects each load cell to its own specific signal conditioner (GEN 5). The same camera as before is set as the master camera. The clock signal from this camera is sent to the signal conditioners and split using a Daisy chain. Both GEN 5 box connects to the computer using an USB connection, sending the time stamped load cell data for Cortex calculations. 


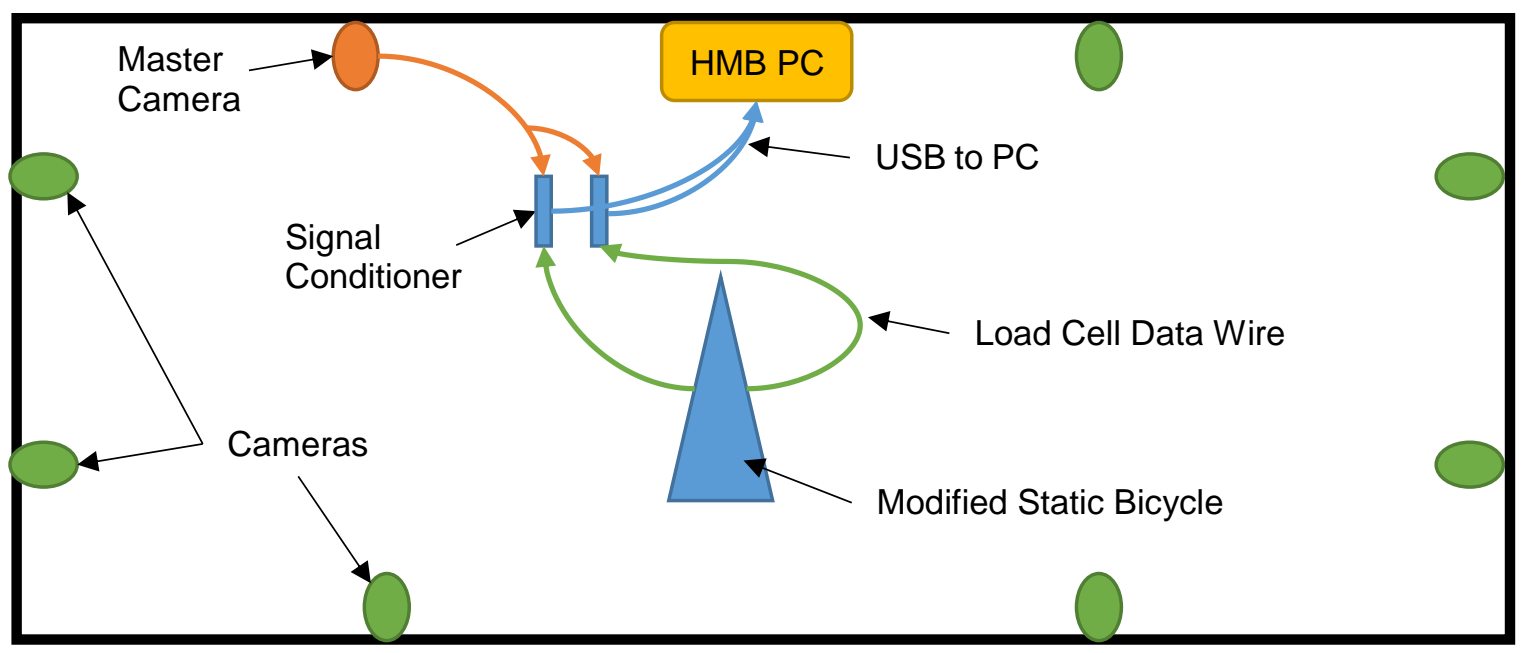

Figure B.20 Set up and connections needed to run a cycling biomechanics experiment.

\section{B.7.3 Final Set Up}

Initial testing demonstrated that running the system as shown in figure B.20 does not allow for Cortex to record data (both force plate or load cell). An operational amplifier had to be added to the system. Figure B.21 shows the final equipment connection for experiments (not actual positions in reference to each other). The clock signal from the master camera is split. The ADC uses it to synchronize force plate data. The operational amplifier sends the clock signal to the GEN 5 boxes to synchronize load cell data. The amplifier increases the clock signal voltage, allowing triggering of all data acquisition devices. A more in depth discussion and reasons for this will be presented in the Troubleshooting section in Appendix C.

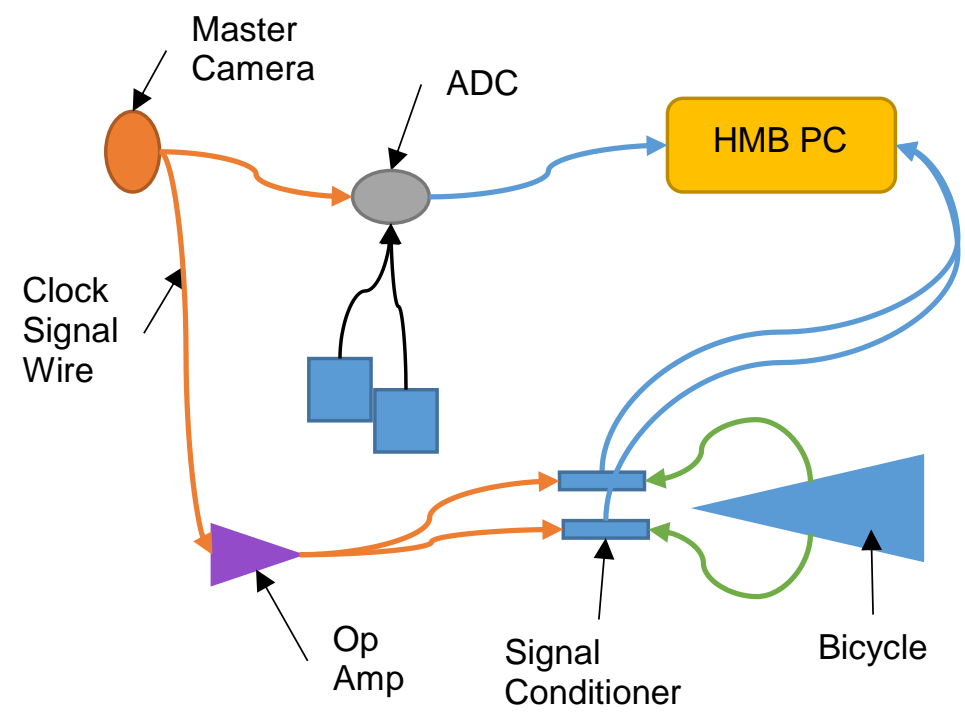

Figure B.21 Equipment connections needed for experiments. Not in actual location as in the lab. 


\section{C: BUILD}

At this point the concept was defined and all parts were selected or designed. The next step was fabricating all parts and assembling the system. The Tioga pedals needed to be modified, while the load cell housing and platforms had to be fully machined. The following discussion describes the processes done. All processes described were done at Cal Poly's student shops: The Hangar and Mustang '60.

\section{C.1 Pedal Modification}

To fit the load cell through the pedal body, the center of the pedal was removed using a pneumatic cutoff wheel. The cuts were deburred with a Dremel. Figure C.1 shows the pedal after machining. Care was taken to not remove material from the outer frame of the pedal as this would decrease the structural strength of the pedal even further. The bearings were removed for this step to avoid chips from going inside the bearings, which may get the bearings stock or cause them to fail prematurely due to increased wear.

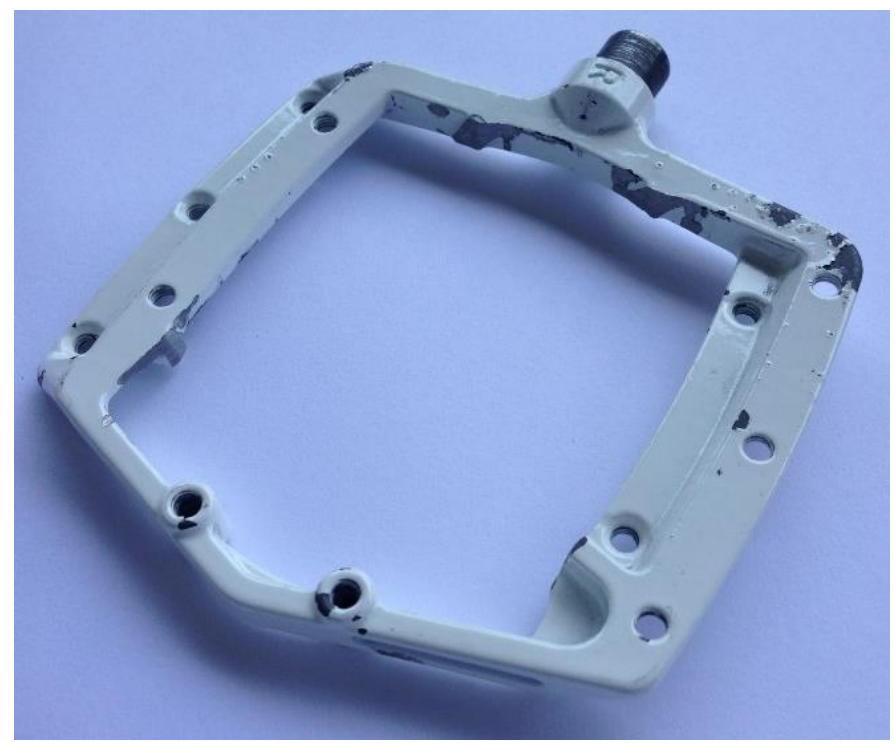

Figure C.1 Pedal after machining. 


\section{C.2 Hole Pattern Fit}

While the vertical mill and mechanical edge finders are accurate tools, they are not designed to located holes. Two tests were done to be sure that the hole positions obtained from the dimensioning are correct. The first test involved laser-cutting the hole pattern in wood as it is most efficient if several tests are needed. The holes had a diameter of 0.150 inches creating a close fit clearance hole. The pedal with 6-32 screws was placed on the holes and the four holes used for attaching the pedal to the housing were checked for fit. Figure C.2 A shows the fit test. The holes circled in red are the holes used for attaching the pedal to the load cell housing. It can be seen that the holes fit the clearance holes. Although the first fit test passed, this does not guarantee fit as these are clearance holes and the real system uses tapped holes. The second test involved drilling and tapping the holes in a piece of aluminum. Once again fit was checked; passing this test would confirm fit for the system. The screws must go into the hole and be able to engage with the threads in the piece of aluminum. Figure C.2 B shows the pedal screwed on the aluminum plate confirming that the hole pattern dimensions are correct. This dimensions can now be confidently used in the actual load cell housing.

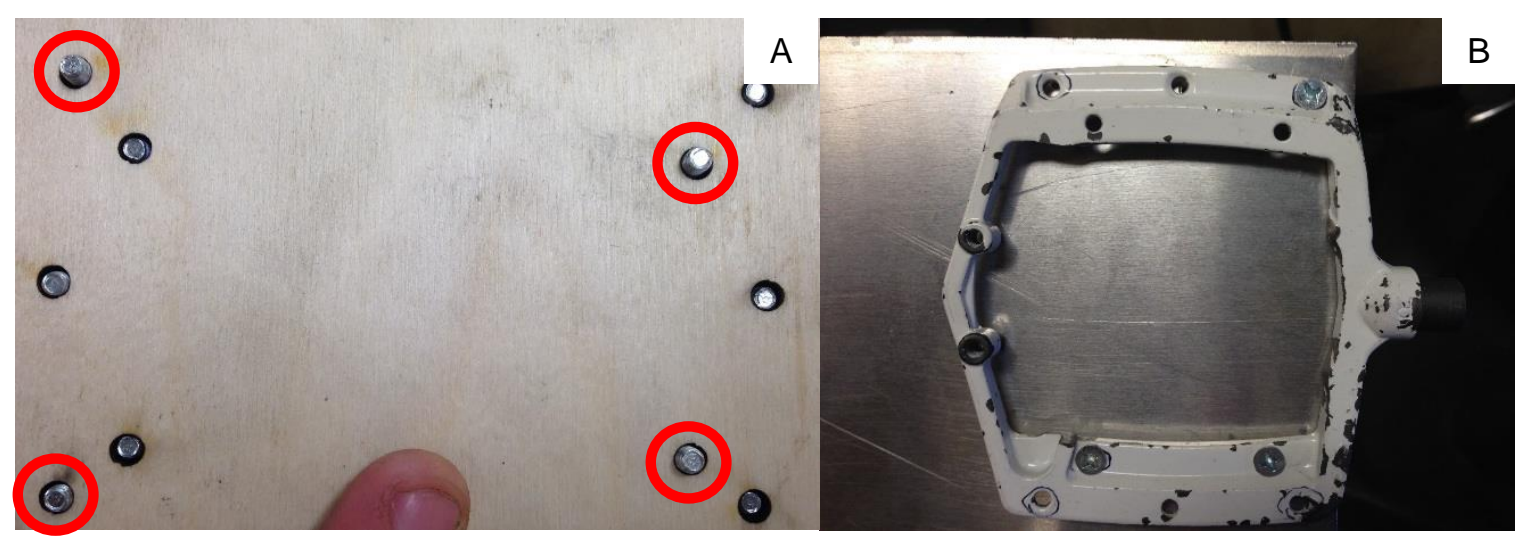

Figure C.2 A.) Wood fit test. Circled holes are used for attaching load cell housing to pedal. B.) Aluminum plate fit test. Pedal is bolted to the plate confirming the dimensions of the holes. 


\section{C.3 Load Cell Housing Machining}

The load cell housing was made from a block of aluminum with dimensions $3 \times 4$ x 6 inches. The raw material was a block of aluminum 6061 (Part 8975K282 McMasterCarr). The block of aluminum was sawed in half with a horizontal band saw. This produced two $3 \times 3 \times 4$ inch blocks. A vertical milling machine was set up with a 0.75 inch end mill to give the load cell housing and the cavity proper dimensions. The vice of the milling machine was checked for alignment so that a movement in the $\mathrm{x}$ or $\mathrm{y}$ direction was purely in the direction expected. The surface that was cut with the band saw was milled off to improve surface quality and to define the height of the load cell. Once the block was 2.150 inches tall, the cavity was machined. A mechanical edge finder was used to locate the edge of the block. The cavity dimensions were measured from the left side of the housing. For all the milling processes a depth of 0.050 inch passes were performed. Figure C.3 shows the aluminum block being machined to form the cavity.

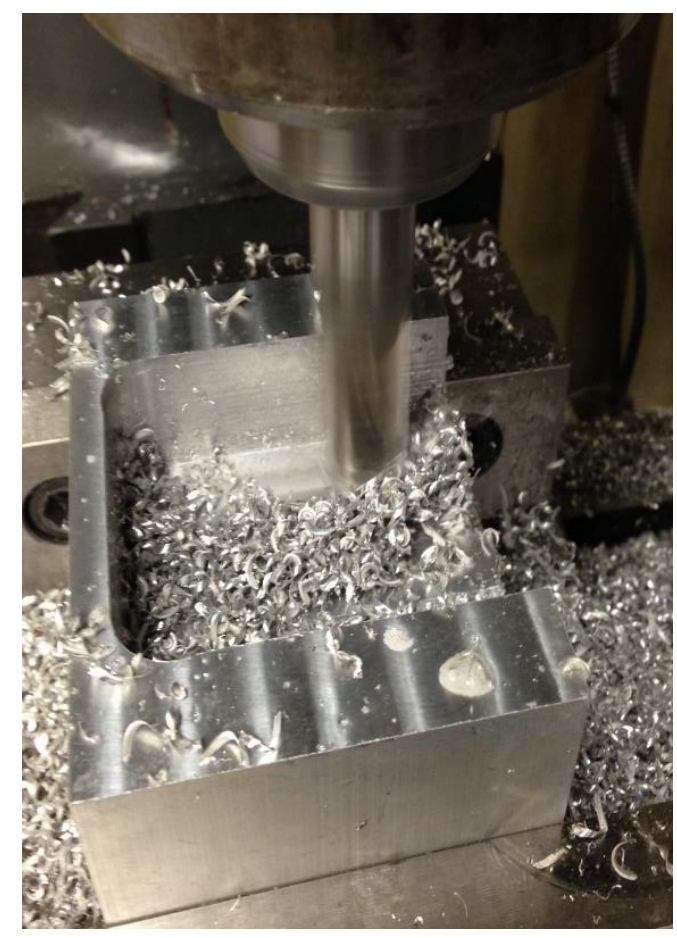

Figure C.3 Pedal housing being machined 
Next, the holes for the 6-32 screws were drilled. These holes were located using using a mechanical edge finder to locate the refernce corner (mentioned in figure B.15). These holes were drilled using a vertical milling machine to accurately position them. A tap guide was used to create an indentation on the block so the drill bit does not move about at the beginning of drilling; this increases the accuracy of the hole locations. A \#36 drill bit was used to make the perforations. Tape was placed at about one inch from the end to quickly show when depth has been reached (figure C.4). The length of these perforations is not critical as it is just providing space for the tap tool. To tap the holes, a 6-32 tapping tool with tapping oil was used. The tap tool was held and pushed vertically by the vertical milling machine. The user only needed to rotate the tap tool to create the threads. The set up is shown in figure C.5. A chamferring tool was used to deburr and create the countersink.

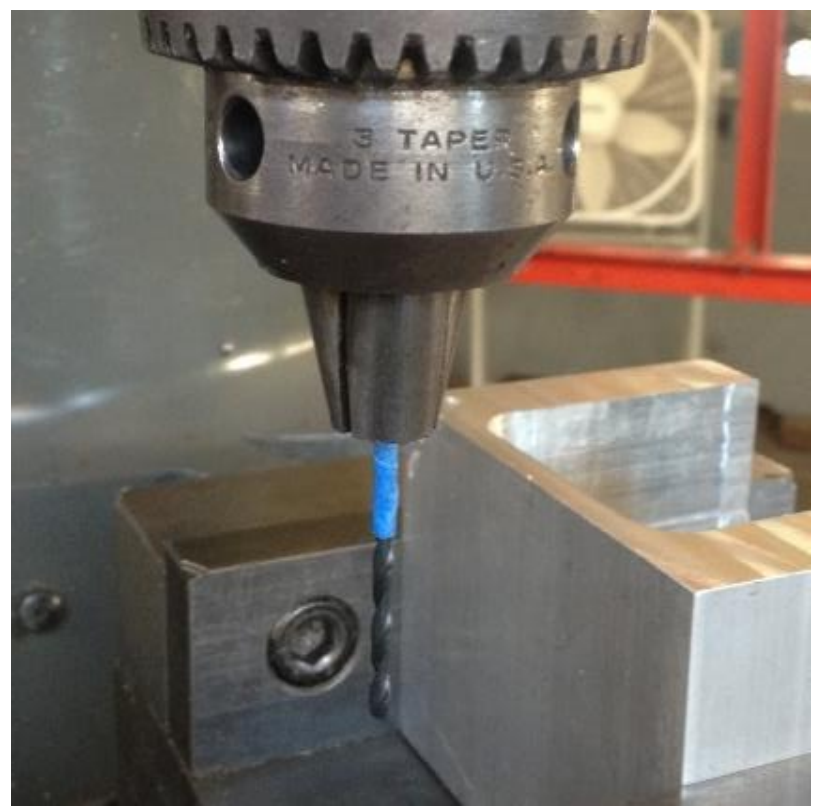

Figure C.4 Drilling set up for pedal attachment holes. Blue tape on drill bit quickly tells depth of perforation.

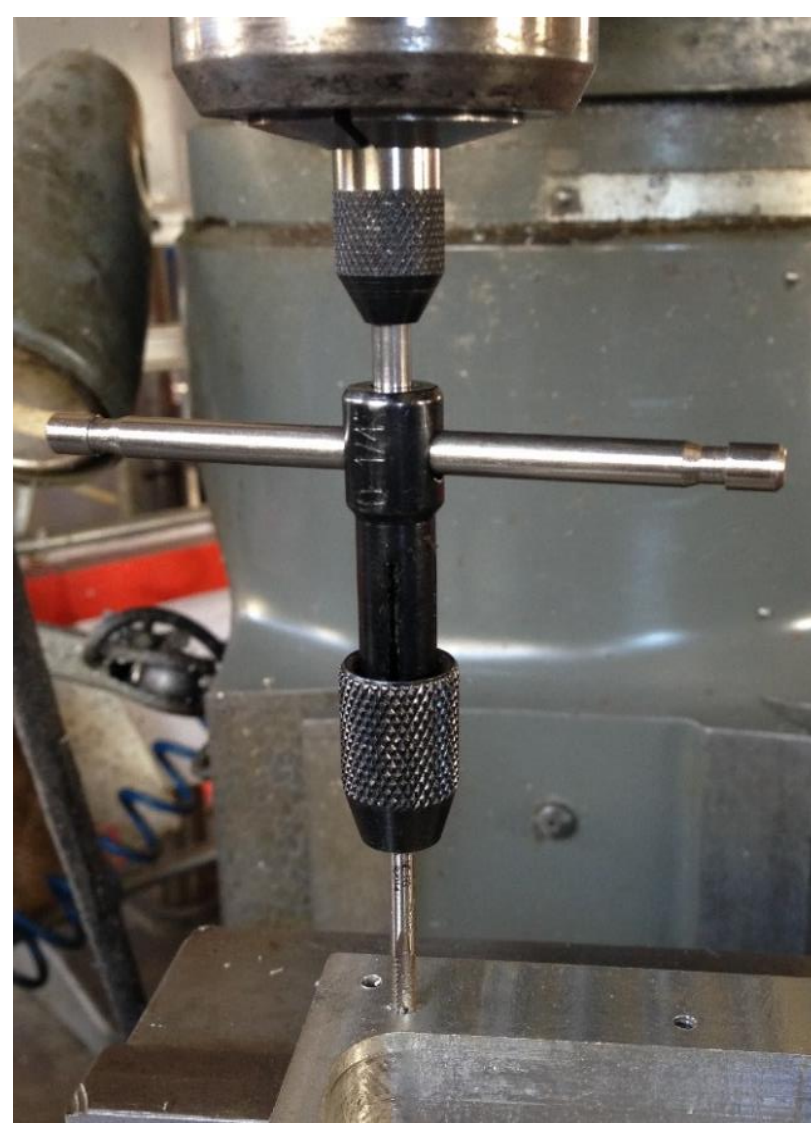

Figure C.5 Thread tapping set up using vertical milling machine. 
The last step is to create the clearance holes for the screws that hold the load cell. The load cell has an eight hole circular pattern with a one inch radius. The screws used to attach the load cell are 10-32 UNF screws. The center of the clearnce hole pattern was located from the reference corner (found in a previous step). The holes are offset in two directions by 0.7071 inches. This creates a square pattern around the center of the cavity. Once made, the holes were deburred using a chamferring tool and a small countersink was made on the outside to aid the insertion of screws. Figure C. 6 shows the finished load cell housing. The total machining time was 5 hours per load cell housing. A majority of the time was spent milling the housing cavity.

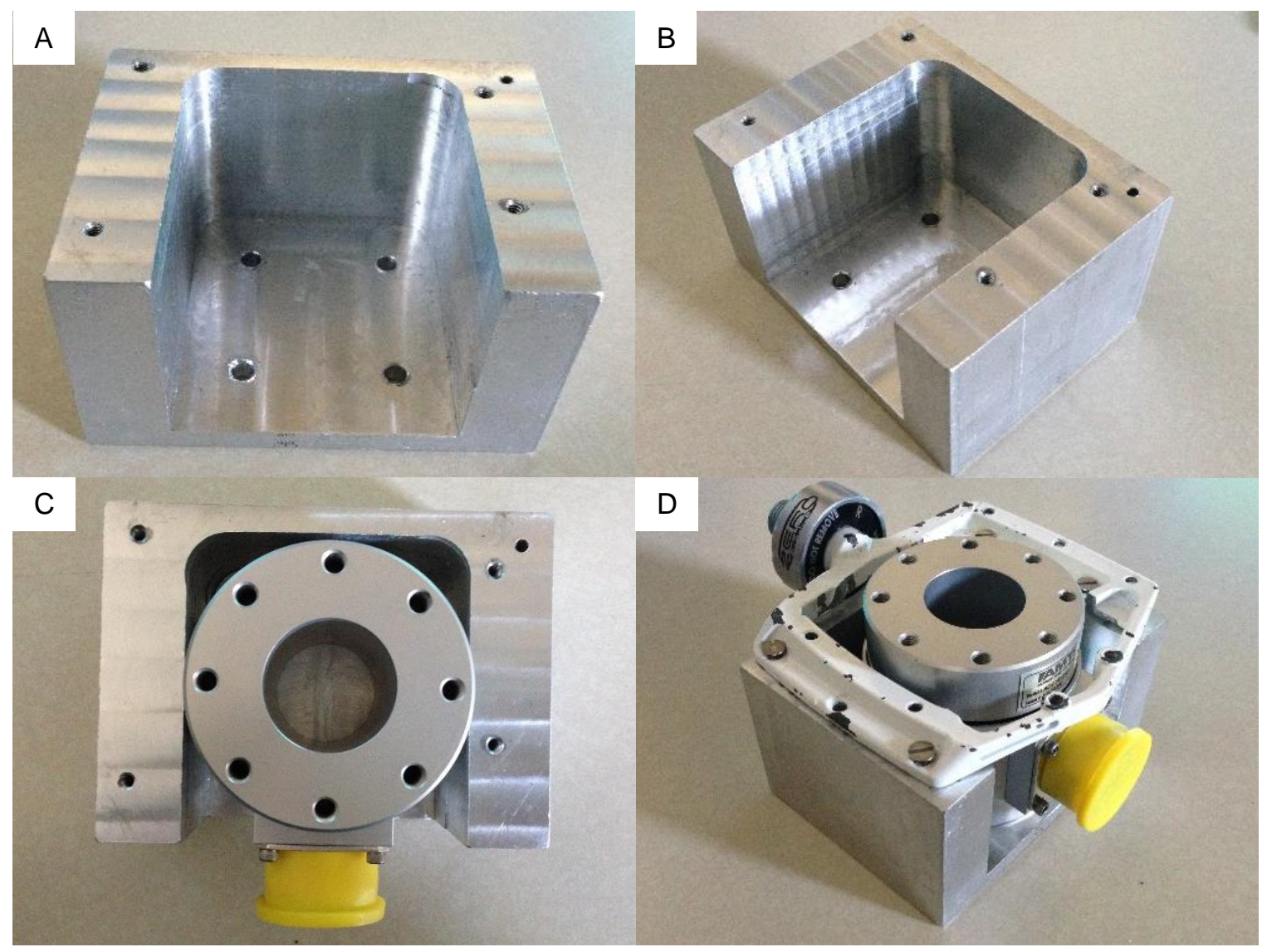

Figure C.6 Finished pedal housing. A.) and B.) show finished pedal housing. C.) shows load cell fit with housing. D.) shows housing, load cell and pedal fit. 


\section{C.4 Pedal Platform Machining}

The platform material bought was a $12 \times 12$ inch plate of aluminum and Buna-N rubber (Part 9525K211 McMaster-Carr). Two 4 × 3.5 inch pieces were cut with a vertical band saw to make each platform. An outline of the desired shape was drawn on the aluminum side of the plate. The foot strap tabs were made by removing 0.25 inch wide strips on both sides of the plate lengthwise, and leaving the material at the locations determined during the design phase (Appendix B.5). The rubber on the tabs was removed, exposing the aluminum plate. This was done to allow the tabs to fit through the pedal straps. A vertical milling machine with a mechanical edge finder was used to locate the center of the hole pattern and to drill the holes used to attach the platform to the load cells. The counterbores were dimentioned to only remove material from the rubber part of the plate. The counterbores hide the head of the screws that attach the platforms to the load cell. Once the holes were finished, the platforms were taken back to the vertical milling machine to round off the corners. Radius of the corner is set to 0.5 inches. The specific radius is not important as it does not provide any fit or feature. The purpose of the rounded corners is to avoid rider injury. The static bicycle has inertia that keeps the pedal rotating if the feet are removed from the pedals. If this occurs, having a square corner of metal flying around becomes a hazard. The rounded corners could prevent cuts to the rider. As a final step, beads of aluminum were TIG (Tugnsten Inert Gas) welded at the edge of the strap tabs. These extra material creates a mechanical lock for the pedal straps. Figure C.7 shows the finished pedal platfrom.

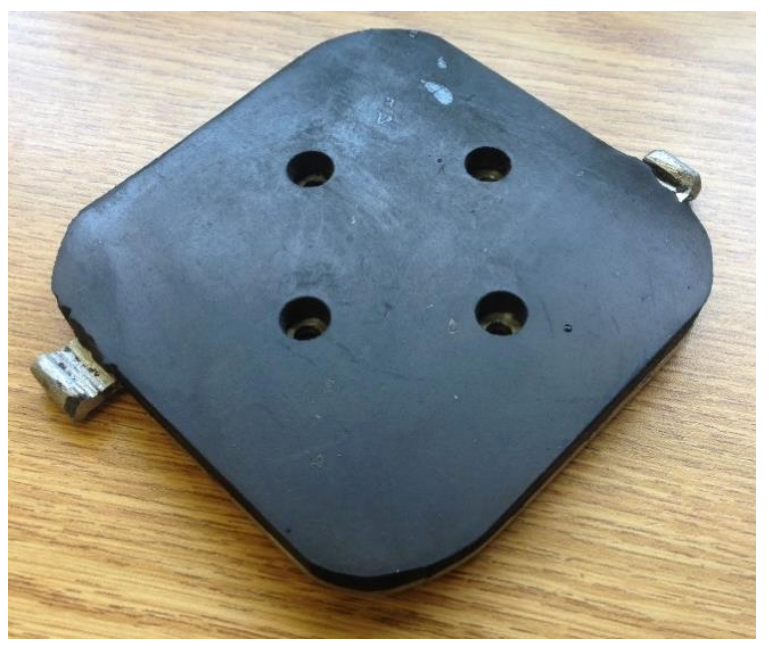

Figure C.7 Pedal platform with foot strap. 


\section{C.5 Final Assembly}

With all the pieces machined, the whole assembly was put together as described in the design part of this chapter (Appendix B.6). The load cell was attached to the load cell housing with four 10-32 screws using a 1/8 inch Ellen wrench. Then, the pedal with the bearing was attached to the housing with four flat head 6-32 screws. Figure C.8 A shows the assembly up to this point. Next, the pedal platform was attached to the top surface of the load cell using four 10-32 screws. The pedal strap was added by forcing the straps over the aluminum beads welded on the tabs. Last, the pedal assembly was mounted on the static bicycle. Figure C.8 B shows the finished right pedal assembly and mounted on the upright static bicycle.

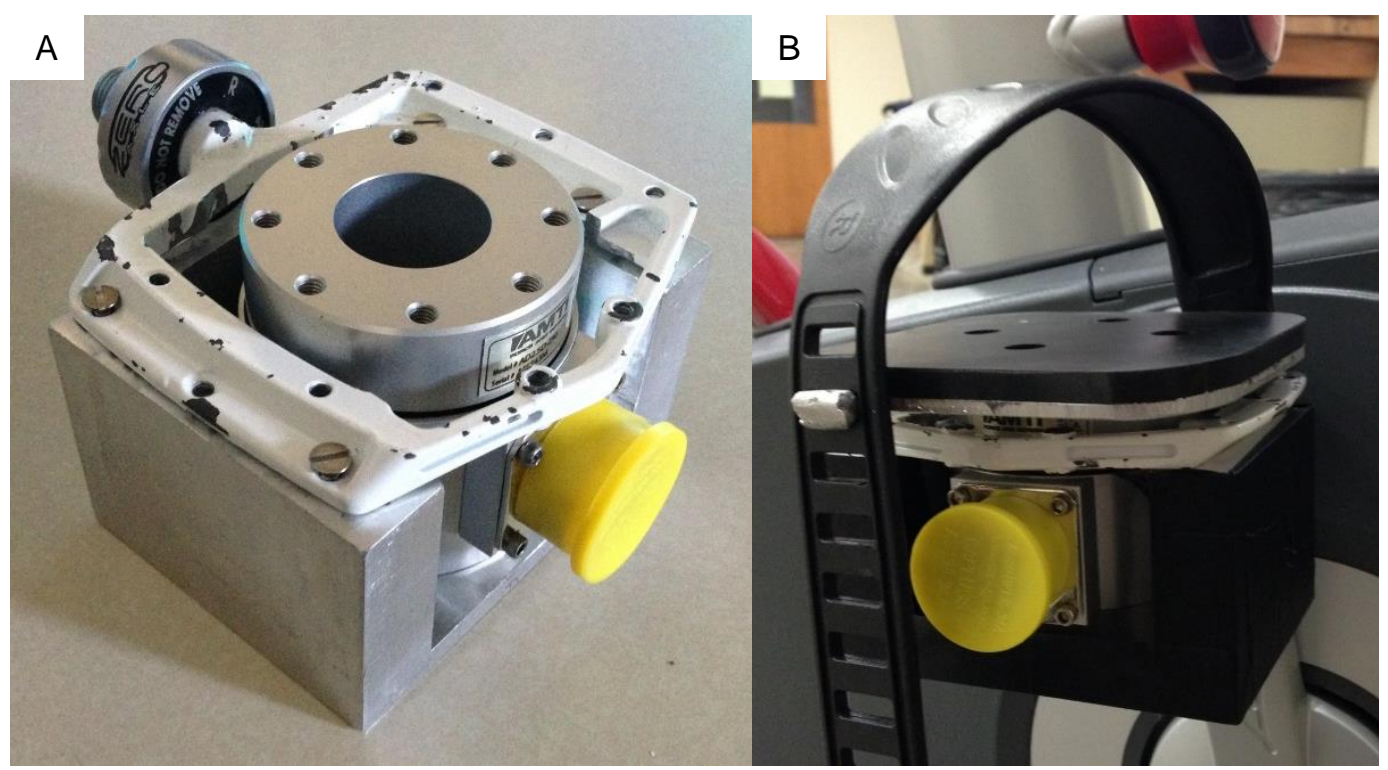

Figure C.8 A.) Housing attached to the pedal and load cell. B.) Finished right pedal assembly. 


\section{C.6 System Integration}

The load cells and amplifiers were connected as mentioned in the Final Set Up section of the Design phase (Appendix B.7.3). To calculate knee loads, Cortex requires the location and orientation of the load cells. To do this, the $\mathrm{HH}$ marker set was modified to add markers for the pedals. Five markers were added to each pedal. Table C.1 lists all the markers used for cycling biomechanics. Markers $1-19$ remain the same as described above (Appendix B.3.1). The new marker positions are shown in figure C.9. The Front, Mid, Back, and Low markers are used to track the position and orientation of the load cell. Cortex is able to create a virtual representation of the load cells based on the pedal marker set and the load cell dimensions given for sizing. The "Track force plate" option is used to follow the virtual load cell segment. This way, the loads recorded can be located in the coordinate system and applied at the feet of the rider to solve the inverse dynamics problem for internal knee joint loads.

Table C.1 Marker list for the cycling marker set based on the Helen Hayes marker set.

\begin{tabular}{|c|l|c|l|}
\hline No & \multicolumn{1}{|c|}{ Name } & No & \multicolumn{1}{|c|}{ Name } \\
\hline 1 & R.ASIS & 16 & R.Knee.Medial \\
\hline 2 & L.ASIS & 17 & R.Ankle.Medial \\
\hline 3 & V.Sacral & 18 & L.Knee.Medial \\
\hline 4 & R.Thigh & 19 & L.Ankle.Medial \\
\hline 5 & R.Knee & 20 & L.Front \\
\hline 6 & R.Shank & 21 & L.Mid \\
\hline 7 & R.Ankle & 22 & L.Back \\
\hline 8 & R.Heel & 23 & L.Low \\
\hline 9 & R.Toe & 24 & L.Spindle \\
\hline 10 & L.Thigh & 25 & R.Front \\
\hline 11 & L.Knee & 26 & R.Mid \\
\hline 12 & L.Shank & 27 & R.Back \\
\hline 13 & L.Ankle & 28 & R.Low \\
\hline 14 & L.Heel & 29 & R.Spindle \\
\hline 15 & L.Toe & & \\
\hline
\end{tabular}




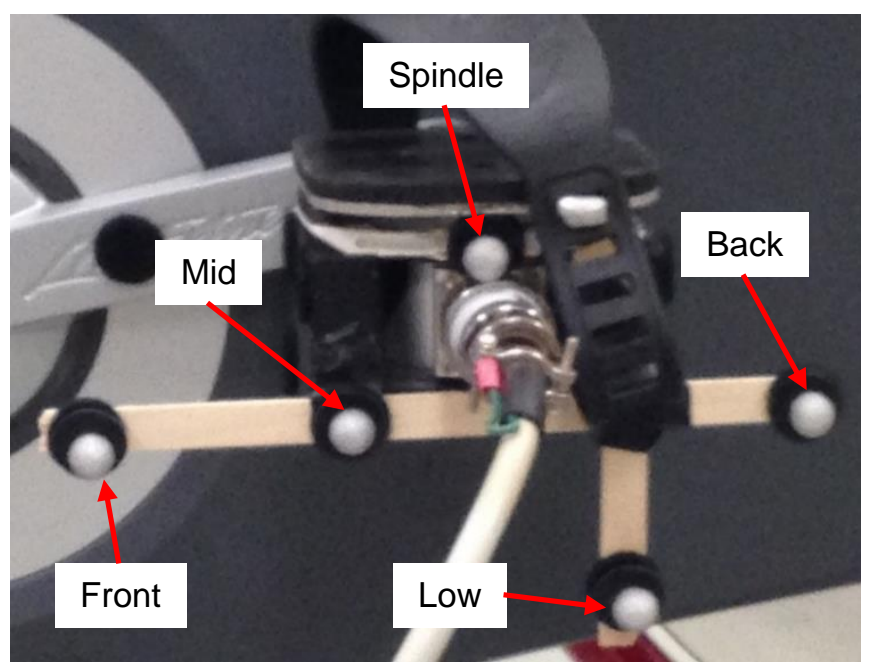

Figure C.9 Pedal marker set.

The Spindle marker is located at the axis of rotation of the pedal (where the spindle would be if the pedal had one). The position of this marker in the global coordinate system is used to calculate the crank angle at which the load cell is at a determinate frame. A detailed discussion on this process is presented in the MATLAB code description later in this chapter (Appendix D.2). The position of the other four markers was selected randomly as they do not represent specific body segments. It was desired that one direction had more markers so it is easier to recognize the pattern when naming markers in Cortex. The markers span farther than the body of the load cell housing to separate them out and avoid marker confusion. Marker confusion occurs when two or more markers are too close together. This causes the camera system to be unable to distinguish the markers from each other and either mixing their paths or fuse them into one marker. Marker confusion makes data difficult to process, and in extreme cases it makes the capture unusable.

At this point the system has all the required elements to run a biomechanics experiment. The system is able to record kinematic and kinetic data, synchronize it, and solve the inverse dynamics problem to estimate internal knee loads (forces and moments) in the coordinate system of the tibia. This data can then be taken for post-processing. 


\section{C.7 Troubleshooting}

As expected for any new system, there were some issues that had to be sorted out. The following discussion describes these issues, the cause, and the steps taken to resolve them. The system was fully functional after these issues were addressed.

\section{C.7.1 Clock Signal}

Cortex documentation showed the clock signal being directed from the master camera into the GEN 5 signal conditioner and split with a Daisy chain (see figure B.20). This set up did not allow for the recording of any data. The amplitude of the clock signal was checked with an oscilloscope (Agilent 54622A) at different locations of the signal path. It was found that when the load cells are not connected and the clock signal is not sent to the GEN 5 conditioners, the clock signal amplitude was $2.4 \mathrm{~V}$. This is enough to trigger the ADC. When the full system is connected, the clock signal amplitude decreased to 860 $\mathrm{mV}$ at all connection places. This voltage is far too low to trigger either the ADC or the load cells, explaining why Cortex could not record any data.

There are two issues at play here. First, the Owl cameras only output a clock signal amplitude of $3 \mathrm{~V}$; insufficient to trigger the load cells but enough for the ADC. Second, the reduction in voltage occurs due to the high impedance from the GEN 5 boxes which brings the whole signal too low for any triggering to occur. To solve these issues an operation amplifier (Daedalon Corporation EG-02) was added to the system. The camera clock signal is split between the ADC and the operational amplifier (op-amp). The gain setting in the op-amp is set to get a signal between $5 \mathrm{~V}$ to $10 \mathrm{~V}$ to trigger the load cells. The opamp serves as a separation between the camera and GEN 5 boxes. This keeps the ADC voltage at $2.4 \mathrm{~V}$, triggering the ADC at a safe level (under $5 \mathrm{~V}$ ). Figure $\mathrm{C} .10$ shows the set up and the voltages found at each segment of clock signal path. 


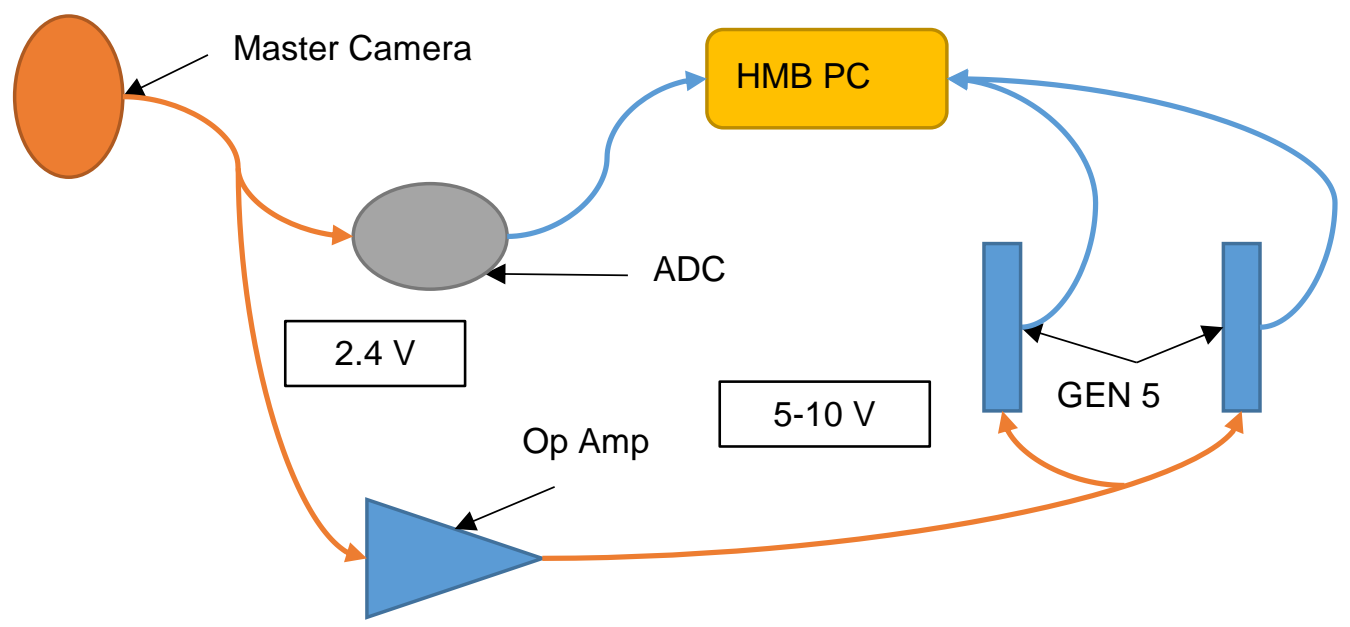

Figure C.10 Op-amp set up to fix triggering issue with clock signal.

\section{C.7.2 Data Quality}

Data smoothness is very important for the accuracy of the calculated knee loads. It is imperative that load cell data has no noise or gaps as these irregularities will propagate throughout the calculations, concluding with inaccurate results. During initial testing strange loads were being obtained from Cortex (figure C.11 A). A smooth curve was expected, however the results where noisy and had sections randomly approaching zero. These loads were repeatable in several trials for all subjects. The cause of these incorrect calculated loads was the load cell data recorded (figure C.11 B). The load cell data seems to follow a smooth curve but randomly loses all data (recorded zero loads). At first, it was thought this was a cable connection issue as the data loss
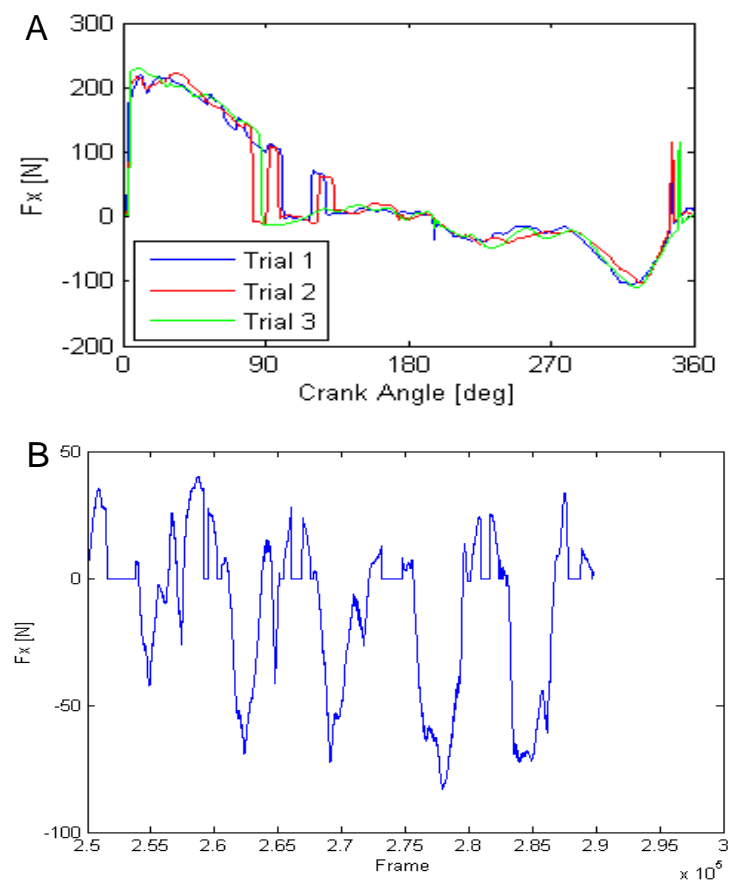

Figure C.11 Bad quality data from initial testing. A.) Bad knee loads is repeated during several trials. B.) Load cell data missing parts. 
seemed to occur at the same crank angle range. The short duration of each data loss segment also suggested a lose wire coming in and out of connection. However, after several tests it was discovered that the reason for the data loss was the Center of Pressure (COP) filter. Cortex uses several filters to smooth data. One load cell data filter cases any load below a threshold value to not be recorded (Threshold filter). The second filter forces all measured loads to zero when the COP of the load recorded by the load cell is placed outside of the virtual body of the load cell. The load cells are able to calculate the COP of the load applied on them based on the force and moment levels recorded. Cortex uses this position to place a force vector in the virtual representation of the lab space. This way, Cortex can locate the load cell measurements with respect to the subject and apply these loads to the appropriate body segment.

Because the load cell dimensions given to Cortex were $2.5 \times 2.5$ inches (actual dimensions of the load cells), the force vector was being placed outside the virtual body of the load cells at times. The pedal platform ( $4 \times 3.5$ inches) is the actual force application surface and it is bigger than the top surface of the load cells. Therefore, a force applied at an edge of the pedal platform would be placed outside this $2.5 \times 2.5$ inch area and would be zeroed out by Cortex.

The solution involved increasing the size of the load cell in Cortex. Several test were done to find the optimal load cell dimensions that would reduce or eliminate the data loss. It was found that making the load cell larger than $6 \times 6$ inches $(15 \times 15 \mathrm{~cm}$ as inputted in Cortex) did not improve the issue any further. For completeness, several settings were tested for the threshold filter, however, eliminating this filter deteriorated data quality greatly. It was concluded that a $5-10 \mathrm{~N}$ threshold distorted the data the least amount. Putting this new values improved the data quality but did not fully fix the issue. However, it allowed enough data to be smooth enough to proceed with the project. 


\section{D: $\quad$ MATLAB CODE}

Cortex outputs data in different formats. Marker positions are exported using a Track Row Column (TRC) or ".trc" files [25]. The calculated knee joint forces are exported using a DATA or ".data" file [25]. Both files can only be used by Cortex or opened in Excel as a text file. Manipulation of this data is needed to get the format and post-processing required. This manipulation is done in MATLAB (MathWorks). The MATLAB code must be able to edit the TRC and DATA files, bring all the data into the MATLAB workspace and perform all calculations needed to output the results in the desired format. The gait and cycling code are slightly different to each other. The following discussion describes the MATLAB code created and the calculations performed.

\section{D.1 Inputting Data into MATLAB}

Cortex outputs marker position data in TRC files. The calculated knee loads are outputted as a DATA file. Figure D.1 shows how both files are seen when opened in Excel. To get this data in MATLAB in a usable form, the first few rows must be removed.

A.) DATA file format

\begin{tabular}{|c|c|c|c|c|c|c|c|c|c|}
\hline \multicolumn{10}{|l|}{ [Data] } \\
\hline & \multicolumn{2}{|c|}{ HipJointAngle } & & & & & \multicolumn{2}{|c|}{ KneeJointAngle } & \\
\hline & R Hip FE & \multicolumn{2}{|c|}{ R Hip Abd. R Hip IRER } & RL Hip FE & \multicolumn{2}{|c|}{ L Hip Abd/L Hip IRER } & R Knee FE & R Knee Va & R Knee IRI \\
\hline Index & Series & Series & Series & Series & Series & Series & Series & Series & Series \\
\hline 1 & 48.36266 & -20.3232 & -7.17376 & 46.11104 & -13.4785 & -23.1642 & 79.03487 & -11.7015 & 2.036858 \\
\hline 2 & 48.35286 & -20.2519 & -7.11434 & 46.11814 & -13.5453 & -23.2139 & 78.9521 & -11.7322 & 2.015509 \\
\hline 3 & 48.45199 & -20.1889 & -7.02745 & 46.12458 & -13.6064 & -23.2588 & 79.01057 & -11.7232 & 2.007462 \\
\hline 4 & 48.48953 & -20.1353 & -6.96659 & 46.12993 & -13.6586 & -23.2953 & 79.00032 & -11.7329 & 1.995728 \\
\hline \multicolumn{10}{|c|}{ B.) TRC file format } \\
\hline PathFileT & \multicolumn{2}{|c|}{$4(X / Y / Z)$} & \multicolumn{7}{|c|}{ G:\Experimental Data \2015\11-23\2015Nov23-2\2015Nov23-2_Cycling1_F } \\
\hline DataRate & \multicolumn{2}{|c|}{ CameraRa NumFram } & NumMark & k Units & OrigDataR & OrigDataS & OrigNumF & Frames & \\
\hline 150 & 150 & 9120 & 29 & $\mathrm{~mm}$ & 150 & 1 & 9120 & & \\
\hline \multirow[t]{2}{*}{ Frame\# } & Time & R.ASIS & & & L.ASIS & & & V.Sacral & \\
\hline & & $\mathrm{X} 1$ & Y1 & $\mathrm{Z1}$ & $\mathrm{x} 2$ & Y2 & $\mathrm{Z2}$ & $\mathrm{x} 3$ & Y3 \\
\hline 1 & 0 & -134.359 & 592.2861 & 1273.181 & 100.9375 & 604.0112 & 1291.64 & -18.2688 & 790.3498 \\
\hline 2 & 0.007 & -134.367 & 592.2859 & 1273.192 & 100.9567 & 603.9762 & 1291.666 & -18.0093 & 790.5668 \\
\hline 3 & 0.013 & -134.373 & 592.2858 & 1273.2 & 100.9746 & 603.9374 & 1291.693 & -17.7675 & 790.768 \\
\hline 4 & 0.02 & -134.376 & 592.286 & 1273.203 & 100.9898 & 603.8909 & 1291.72 & -17.5561 & 790.9416 \\
\hline
\end{tabular}

Figure D. 1 Cortex output files format. A.) DATA format. B.) TRC file format. 
Existing code from the HMB Lab was modified to open both files, find the end of each line and delete all characters in the lines that are not needed. This file saved all the data as a structure in MATLAB. The code saved the column names as variables names for each data set. As MATLAB uses periods to denote structures, the column with periods in their names must be modified. The code replaced all periods with underscores. For example, "R.ASIS" is modified to "R_ASIS". This data would be saved in "RAW_TRC.R_ASIS" where "RAW_TRC" is the name of the structure and "R_ASIS" is the variable name containing three columns of data for the $\mathrm{X}, \mathrm{Y}$, and $\mathrm{Z}$ positions. Likewise, MATLAB does not use spaces in variables names so these must be changed as well. In DATA files, the spaces in column names get replaced with underscores. Again, this data is saved as structure, therefore, "R Hip FE" is saved as "RAW_DATA.R_Hip_FE".

\section{D.2 Cycling Code}

A set of MATLAB functions were created to modify cycling data and output it with respect to crank angle. The user must input the name of the DATA and TRC files, the mass of the subject, and the frames to get data from. The mass of the subject is needed as Cortex normalizes force and moment data dividing by the mass of the subject. It is expected that one recording is done for cycling data with several crank cycles. Only three cycles are used to obtain the data. The frame range given by the user will have data for at least three full cycles that will be used for processing. Other data needed for the code are names of published data for comparison, names for output files, and naming of dominant leg of the subject. 
Data is inputted into MATLAB using the scripts mentioned previously. The code uses the frame range given to copy the data into a new matrix. This matrix has the format shown in Table D.1. This format is expect for all subsequent code. The names signify the direction of load when positive. Power is generated when the values are positive and absorbed if the values are negative. Note that the study did not used the power values but the code is able to perform all calculations on the knee power data from Cortex. The crank angle requires calculations with $\mathrm{TRC}$ data.

Table D.1 MATLAB cycling code expected data format.

\begin{tabular}{|c|c|l|}
\hline Column & Variable & \multicolumn{1}{|c|}{ Name } \\
\hline 1 & $\theta$ & Crank Angle \\
\hline 2 & $\mathrm{Fx}$ & Anterior Force \\
\hline 3 & $\mathrm{Fy}$ & Lateral Force \\
\hline 4 & $\mathrm{Fz}$ & Compression Force \\
\hline 5 & $\mathrm{Mx}$ & Valgus Moment \\
\hline 6 & $\mathrm{My}$ & Extension Moment \\
\hline 7 & $\mathrm{Mz}$ & $\begin{array}{l}\text { External Rotation } \\
\text { Moment }\end{array}$ \\
\hline 8 & $\mathrm{VV}$ & Valgus Angle \\
\hline 9 & $\mathrm{FE}$ & Flexion Angle \\
\hline 10 & $\mathrm{IE}$ & Internal Rotation Angle \\
\hline 11 & $\mathrm{PS}$ & Sagittal Plane Power \\
\hline 12 & PF & Frontal Plane Power \\
\hline & & $\begin{array}{l}\text { Transverse Plane } \\
\text { Power }\end{array}$ \\
\hline 13 & PT \\
\hline
\end{tabular}


The crank angle is calculated using the position of the spindle marker. The maximum and minimum values of the spindle position in the vertical and horizontal planes are found. The center of the circular spindle trajectory is if calculated by taking an average of the maximum and minimum values in the horizontal and vertical plane. The position of the center of the spindle corresponds to the position of the center of the crank in the global coordinate system. The crank position is subtracted from all spindle marker position data, essentially moving the spindle path around the origin of the coordinate system. At this point, an inverse tangent function turns the two dimensional data into angles, given crank angle with reference to the horizontal positive axis. Published data is referenced to the vertical and increases in clockwise direction, opposite of the tangent results (see figure D.2). To match this format, the crank angle vector is multiplied by negative one (inverting about horizontal axis). To ensure a range from 0 to 360 degrees, any negative numbers were made positive by adding 360. To "rotate" the vector, 90 was added to the all data points. This made the all angles match the proposed format. Any number above 360 had this value subtracted to ensure the $0-360$ range.

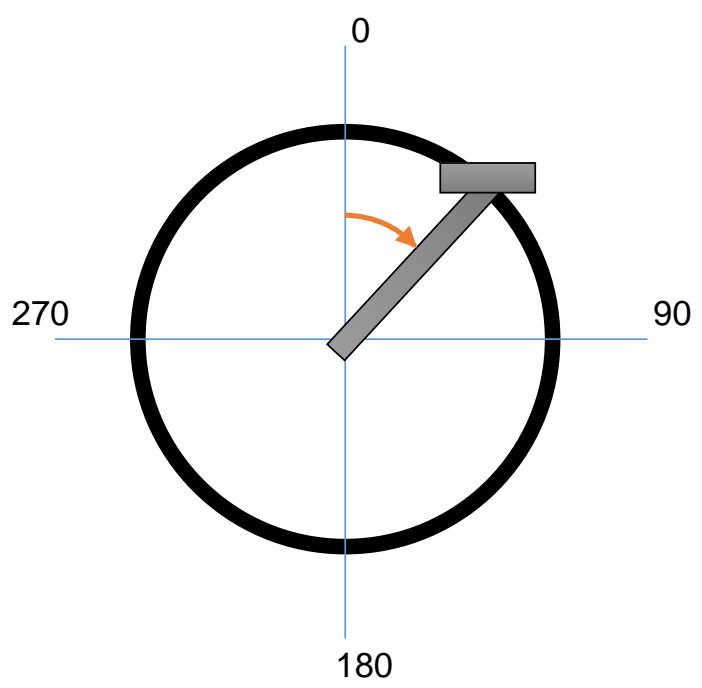

Figure D.2 Crank angle format.

With the crank angle calculated and the data matrix set up, the data must be interpolated for averaging. Averaging at the same crank angle is required for all subjects and for all trials. In cycling, each crank cycle (from 0 to 360 degrees) is a trial and three trials are needed for averaging. Since the cameras may record the pedal at similar, yet different crank angles interpolation needs to be done. A function was created to recognize 
the start ( 0 degrees) and end (360 degrees) of a cycle in the data matrix, take this cycle data onto a different variable for interpolation. A data point before and after this range is needed to interpolate the first and last points. To allow for interpolation at 0 and 360 degrees, the data point before is converted to a negative angle while the data point after is converted to be higher than 360 . Interpolation is done every 0.5 degrees, creating a new vector of the same format as mentioned in table 2.6. Once three matrices are created (one for every trial or crank cycle used), averaging can be done, producing a single set of data. This data set is outputted as a comma separated values file (CSV). Since multiple subjects are used in studies, there will be a set of averaged data for every subject. Lastly, the code makes several plots. The data is plotted for each leg for comparisons between dominant and non-dominant legs. Finally, the code plots all three trials versus crank angle to allow repeatability tests on the data. Figure D.3 shows samples of the code output. The top row shows forces, the middle row shows moments, and the bottom row shows joint angles. 
A
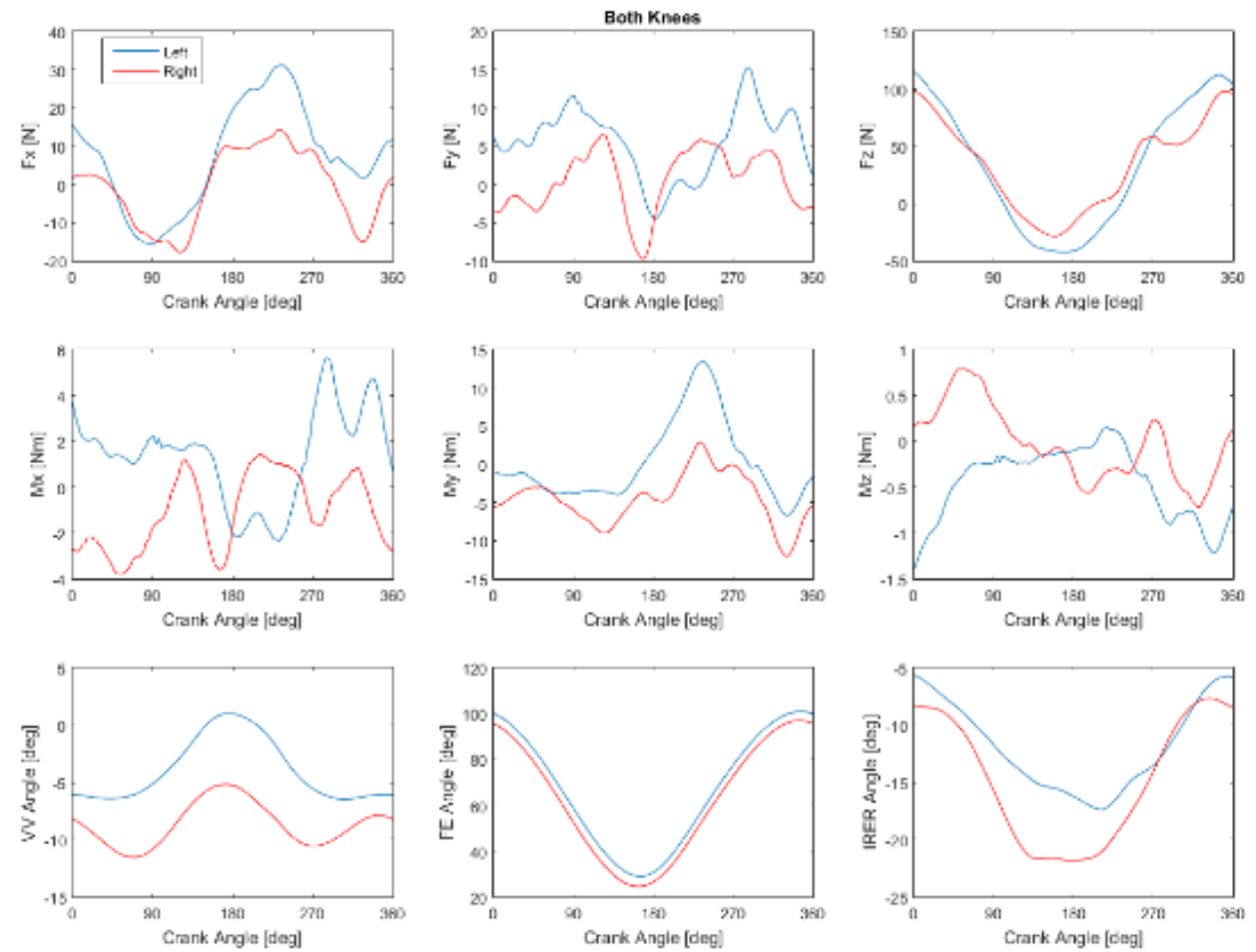

B
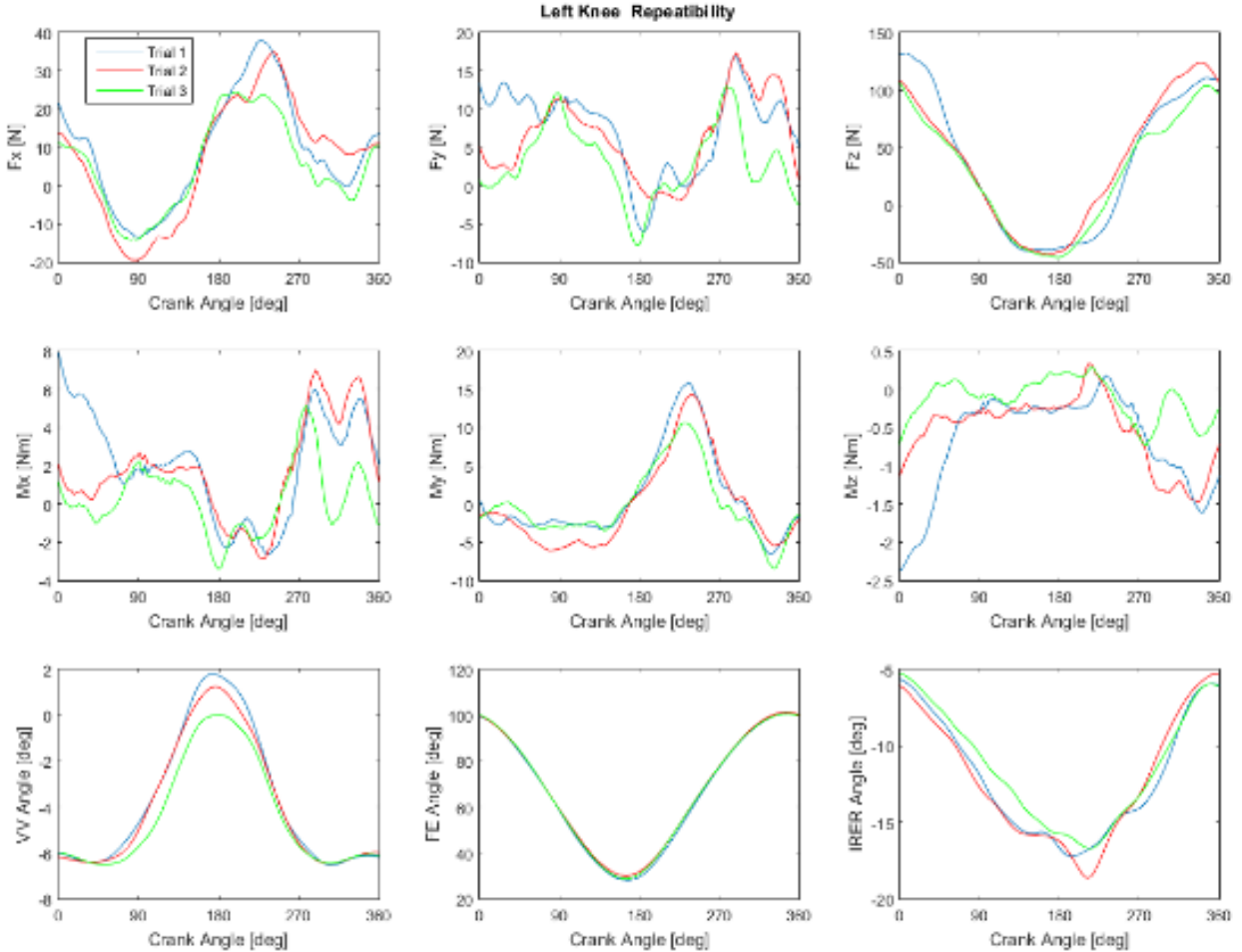

Figure D.3 Cycling MATLAB code plots. A.) Data for both legs plotted against crank angle. B.) Data for three trials plotted to check for repeatability. 


\section{D.3 Gait Code}

The purpose of the system created is to obtain knee loads during cycling. Some experiments involve comparing these loads with gait data. Cortex is used for gait and cycling experiments therefore, data must be processed for both types of experiments. Gait experiments are usually reported as percent stride. Cortex data shows knee loads with respect to time. This MATLAB code must process the gait data similar to the cycling code. One of those differences include outputting data with respect to percent stride instead of crank angle. In cycling, a single motion capture has all the crank cycles needed for processing. In gait, a single capture has a single trial, thus three trials must be recorded to get three sets of data to average.

The cycling MATLAB code was modified to process the gait data. Data input into MATLAB is done the same way as described at the beginning of this section. User input requires mass of subject, leg to use data from, file names of TRC and DATA files, and frames to take data from. These frames are the range from the first hill strike to the next heel strike of the leg to output data for. Percent stride is calculated based on the frames selected. The first frame represents $0 \%$ stride; the last frame is $100 \%$ stride. The data is organized in a new matrix with the format described in table D.2. The load names refer to the direction of the load when its value is positive. Due to different walking speeds and walking styles for different subjects, the cameras may record data at different percent stride values. Interpolation is needed to average data for three trials. The code interpolates the gait data the same way it was done for the cycling code. The interpolated data is outputted as CSV file. The walking speed of the subject is then calculated and reported. The code plots the data for the knee that has a full gait trial captured and processed. Another piece of code opens the CSV files with gait data, averages three trials and creates a new CSV file with the average data for the subject. 
Table D.2 MATLAB gait code expected data format.

\begin{tabular}{|c|c|l|}
\hline Column & Variable & \multicolumn{1}{|c|}{ Name } \\
\hline 1 & $\mathrm{~S}$ & Percent Stride \\
\hline 2 & $\mathrm{Fx}$ & Anterior Force \\
\hline 3 & $\mathrm{Fy}$ & Lateral Force \\
\hline 4 & $\mathrm{Fz}$ & Compression Force \\
\hline 5 & $\mathrm{Mx}$ & Valgus Moment \\
\hline 6 & $\mathrm{My}$ & Extension Moment \\
\hline 7 & $\mathrm{Mz}$ & $\begin{array}{l}\text { External Rotation } \\
\text { Moment }\end{array}$ \\
\hline 8 & $\mathrm{VV}$ & Valgus Angle \\
\hline 9 & $\mathrm{FE}$ & Flexion Angle \\
\hline 10 & $\mathrm{IE}$ & Internal Rotation Angle \\
\hline 11 & $\mathrm{PS}$ & Sagittal Plane Power \\
\hline 12 & $\mathrm{PF}$ & Frontal Plane Power \\
\hline 13 & $\mathrm{PT}$ & $\begin{array}{l}\text { Transverse Plane } \\
\text { Power }\end{array}$ \\
\hline
\end{tabular}

\section{D.4 Population Code}

A population refers to a group of subjects with certain common factors. Once the cycling and gait code has been run, the CSV files outputted by the codes is opened by another MATLAB script. This new code averages data sets for all subjects within a population. The script also finds the maximum and minimum values for every load of each subject and outputs this information in a CSV file. The cycling and gait data printed in the CSV file follow the format described in table D.1 and table D.2.6, respectively. Finally, this code plots the data of both populations for comparison and against published values. The values yielded by the MATLAB code can be used for further processing and statistical analysis. 


\section{E: VALIDATION}

A set of requirements were created at the beginning of the project. These requirements must be met by the system developed. The following section describes the steps taken to validate this list of requirements.

\section{E.1 Rider Kinematics}

To obtain reliable data, the rider kinematics must be kept as close as possible to the kinematics of normal cycling. The pedals of the bike were replaced with a load cell that was positioned using a custom made housing. One test was done to check that this requirement has been met. The test involved asking six volunteers to ride the bicycle and get a feel for it. The volunteers were asked to report if they felt any differences pedaling the modified bicycle compared to any other bicycle they have used before. After pedaling the bike for as long as the volunteers needed, no differences were reported. This confirmed that the modified static bicycle is not intrusive and is expected to keep rider kinematics. The pedal was designed to place the feet of riders at the same location as the original pedal did, and volunteers reported no differences.

\section{E.2 Pedal Mass Effect}

There were concerns that the added mass at the pedals could have negative effects on the riding experience. To make sure this was not the case a test and an analysis were done on steady state cycling. The volunteers from the test mentioned above reported no differences felt while riding the modified static bicycle. This was considered a pass of this qualitative test as it would mean that the volunteers could not feel any difference between normal pedals and the modified pedals with load cells. 
An analysis was made in ADAMS (MSC Software, Newport Beach, CA). This analysis compared torque required to rotate the pedals as the pedal mass changed. The system was modeled by five parts (two pedals, two cranks, and one bottom bracket) and five joints. The joints between the pedal and the crank were "revolute joints" that allow free rotation about the spindle axis. Two "fixed joints" were placed between the cranks and the bottom bracket fixing all degrees of freedom. Another "revolute joint" was added between the bottom bracket and the ground, fixing the model in place but allowing rotation of the bracket about its axis (see figure E.1).

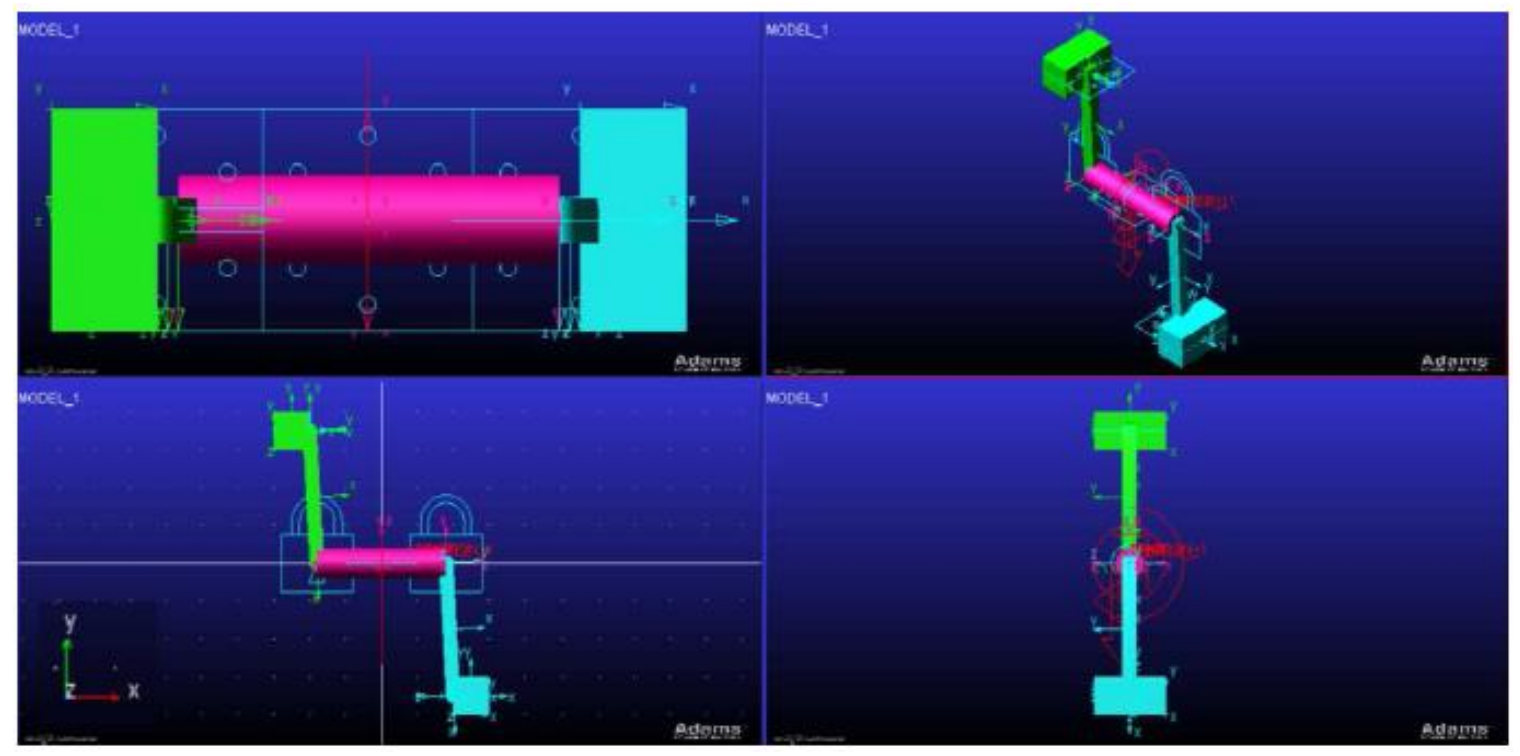

Figure E.1 ADAMS model of the crank and pedal system.

The input was a torque applied at the joint between the bracket and the ground. Static and dynamic friction were applied at the same joint as torque to represent different resistance levels set on the bike. It was assumed that the rider effort is directly proportional to the torque inputted to the system. While the masses and dimensions are not the same of the bike in question (this study was done before the build phase), the behavior should hold true for the system fabricated. 
A PI (proportional-integral) controller was implemented to achieve 50 RPM; allowing all simulations to reach the same angular velocity. This control in angular velocity allows the simulation to provide comparable torque requests to run the model. The system may request more torque than possible for a cyclist to create. This limitation will only affect the transient response of the model and thus is ignored as only steady state is considered. Three simulations were done with different friction settings and pedal masses. The first simulation used $2 \mathrm{~kg}$ pedals. The second simulation used $3 \mathrm{~kg}$ pedals. The third simulation had $3 \mathrm{~kg}$ pedals and half of the value applied to the first two simulations. The model was exported to Simulink where the model behavior was analyzed.

The results are summarized in figure E.2. All simulations reached the same angular velocity (figure E.2 A). Figure E.2 B shows the torque required for the model to reach and maintain the $50 \mathrm{RPM}$. At $39 \mathrm{Nm}$, the $3 \mathrm{~kg}$ pedal needed more torque to be applied at the crank to maintain the same angular velocity than the $2 \mathrm{~kg}$ pedal $(27 \mathrm{Nm})$. However, the $3 \mathrm{~kg}$ pedal with half the friction required the least torque $(20 \mathrm{Nm})$. It was concluded that the increased pedal mass required more torque to maintain steady state, effectively acting as a higher resistance setting on the bicycle.
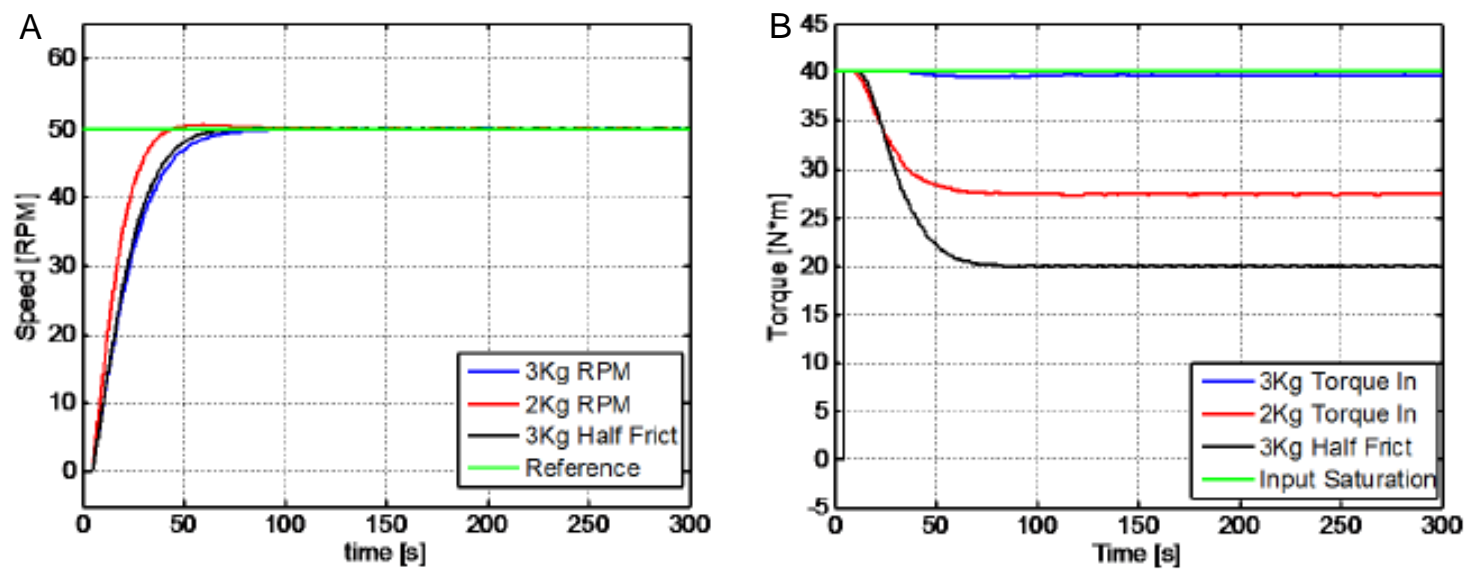

Figure E.2 Results of pedal mass effect in ADAMS. A.) Angular velocity reached by the model. B.) Torque requested by the controller. 


\section{E.3 Measure Loads in Three Dimensions}

To check this requirement, data was recorded and plotted to look for three force components and three moment components. Figure E.3 shows this data. The first row shows force, while the second row plots moments. The first, second, and third column show data for the $\mathrm{X}, \mathrm{Y}$ and $\mathrm{Z}$ directions. Inspection of figure $\mathrm{E} .3$ shows that this requirement is met. Three force and moment components can be recorded with the system.
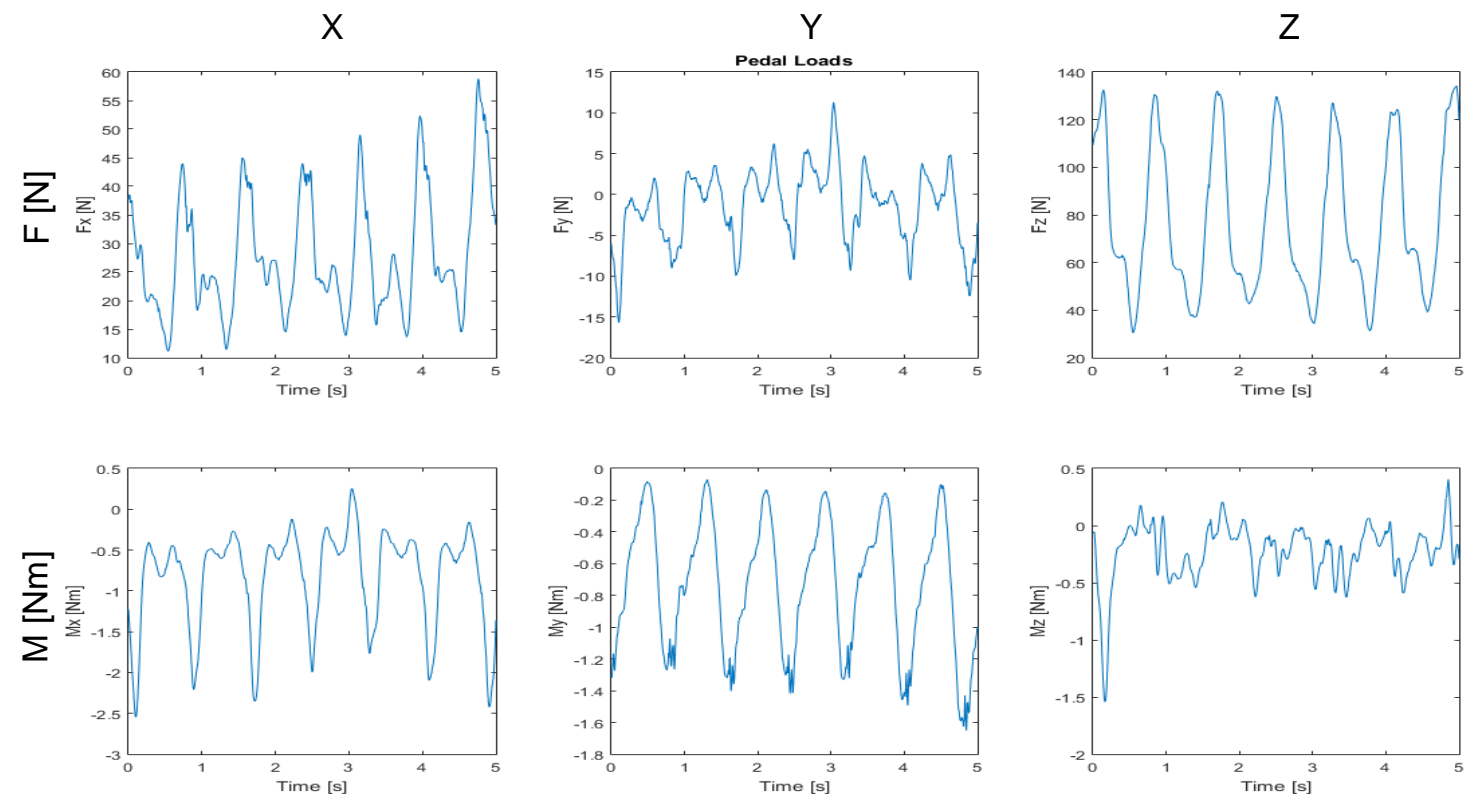

Figure E.3 Pedal data recorded in three dimensions.

\section{E.4 Vibration Isolation}

The GEN 5 signal conditioner boxes should filter the noise out of the load cell data. Therefore, it was assumed that inspection of the data would be sufficient to confirm noise removal from load cell measurements. To check vibration isolation by inspection, data from figure E.3 was checked. As the data is smooth (no spikes, discontinuities or high frequency oscillations) and repeatable for each cycle, it was assumed the signal conditioners removed any noise and vibration artifacts present in the load cell data. 


\section{E.5 Integration with Motion Analysis System}

To test this requirement, a cycling biomechanics test was done. With the system connected as described in Appendix C.6 (System Integration), a cycling experiment was performed. The rider was instrumented with the $\mathrm{HH}$ marker set and asked to pedal at 70 RPM. Kinematic and kinetic data were recorded by Cortex and processed to calculate internal knee loads. In this successful cycling experiment, Cortex recognized the load cells, recorded and synchronized camera and load cell data, and solved for knee loads in three dimensions. The system integration was confirmed.

\section{E.6 Data Output Format}

To report data in standard form, the knee loads and angles must be plotted against crank angle. A MATLAB code was written for this purpose. A full description of this code is found in Appendix D. Data from the experiment mentioned above was processed by the MATLAB code, and knee loads and angles were plotted against crank angle. Inspection of figure E.4 confirms this requirement has been met. Figure E.4 A shows knee forces (first row), moments (second row), and angles (third row) in three dimensions. Figure E.4 $B$ is a close up on the vertical force component to clearly show data plotted against crank angle.

A
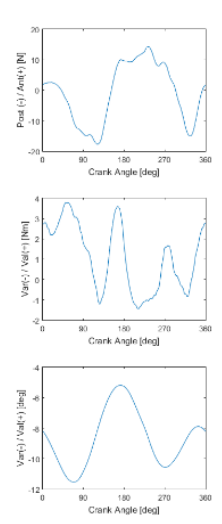
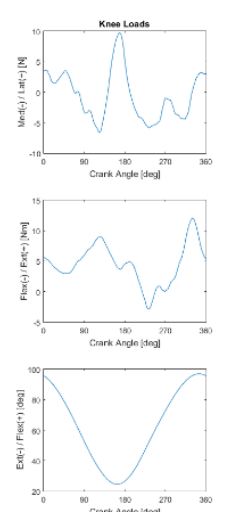
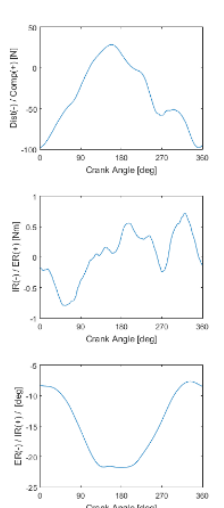

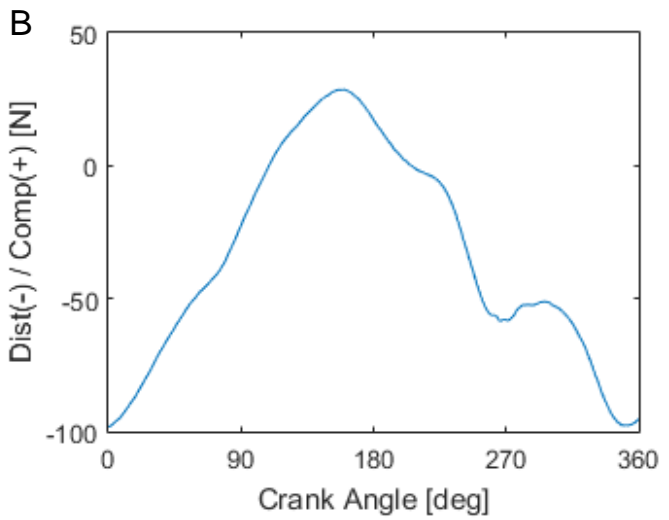

Figure E.4 Cycling data versus crank angle. A.) MATLAB output for all knee forces, moments, and angles. B.) Close up on vertical knee load. 


\section{E.7 Stress Analysis}

The parts fabricated are critical elements of the pedal assembly. The pedals are expected to support the expected loads because they are designed for mountain biking, where cycling while standing up is common. Seated cycling is the only expected used of the modified bicycle. Furthermore, a load of more than $250 \mathrm{lbs}$. should be avoided as it will damage the load cells. No stress analysis is needed for the pedal for these reasons. The load cell housing is a custom made part and validation is required. Stress analysis was performed in the form of a Finite Element Analysis (FEA).

FEA analysis was performed using Abaqus (Simulia, Johnston, RI). This analysis was performed to prove that the custom basket supports the maximum expected loading case by calculating a factor of safety. It is expected that the minimum safety factor is well above 2.65. This number resulted from hand calculations using simple beam theory. The expected worst case scenario is $250 \mathrm{lbs}$. on the longitudinal direction of the load cell, and $125 \mathrm{lbs}$. on the orthogonal directions, as well as moment magnitudes of $250 \mathrm{lbs}$-in on the orthogonal directions and $125 \mathrm{lbs}$-in in the longitudinal direction of the load cell.

\section{E.7.1 Model Development}

The analysis done on this study was a 3D static, linear analysis. To model the system, the sketch tool in Abaqus was used to create a $3 \mathrm{D}$ model of the load cell housing (see figure E.5). It is worth noting that a SolidWorks model was used initially to bring the part to Abaqus but it was difficult to mesh and did not allow model modifications. Material and sections

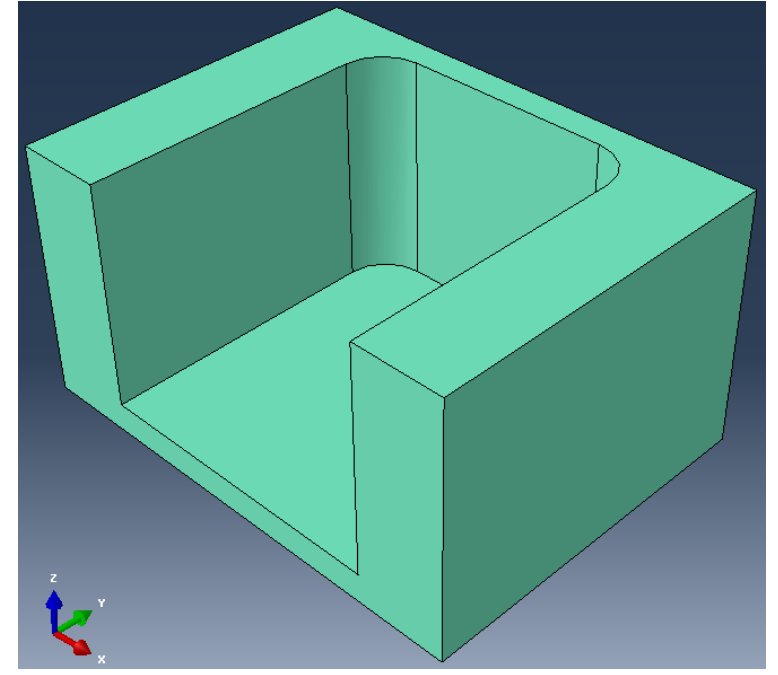

Figure E.5 Load cell housing model in Abaqus. 
definitions were created and assigned to the model. The holes for the screws had to be ignored in this analysis. It was initially planned to stay from modeling the threads of the screws but keep the holes in the model. This complicated the meshing of the model by forcing partitions of the model and complicating load conditions application. Ignoring the holes meant that there was no feature on the model to apply the loads. This was solved by creating datums on the locations of the holes. The bottom plate of the basket was partitioned using these datums to force the creation of nodes at the locations of the bottom plate clearance holes. The loads where applied at these nodes. Figure E.6 shows this partitions done on the bottom plate of the load cell housing.

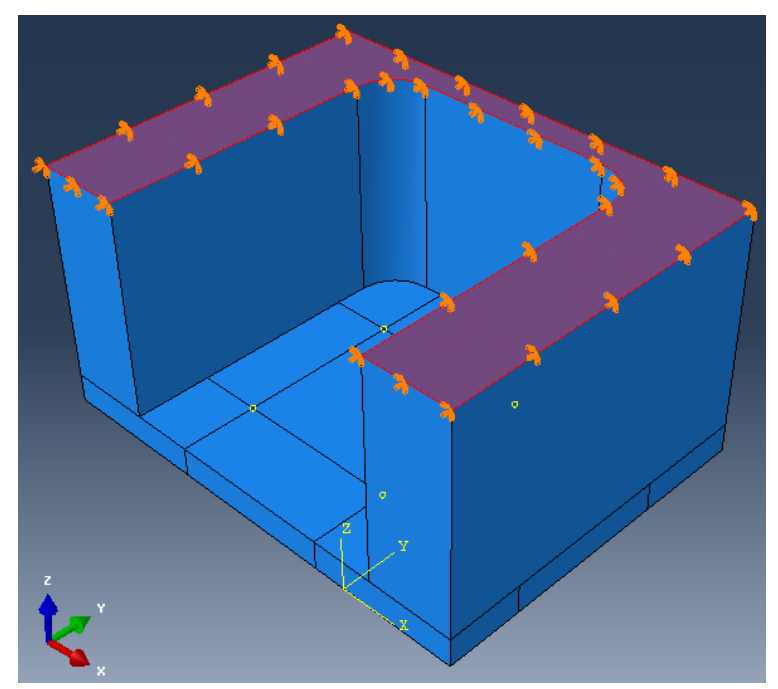

Figure E.6 Partition of load cell housing and boundary conditions applied on top surface.

\section{E.7.1.1Material}

Since the basket was made out of aluminum 6061, this was the material definition created. Under the Mechanical menu, the elastic material behavior was defined by setting Young's modulus to $10.0 \times 10^{6} \mathrm{psi}$ and Poisson's ratio to 0.3 [27].

\section{E.7.1.2Load Conditions}

The basket was modeled with the forces acting at the location of the holes. Initially, the loading was done on a case by case basis. The force on the $x, y$ and $z$ direction were applied individually on the positive and negative directions. Then the moments on the $x$, $y$, and $z$ directions were applied individually on the positive and negative directions. Multiple forces were created to cause the moments on the desired directions. All forces used were concentrated forces applied at a node at the location of the clearance holes. 
Table E.1 lists the magnitude of the forces applied at each node on each loading case. The case of pure forces required one force to be created in Abaqus and applied at four nodes. The moments on the $\mathrm{x}$ and $\mathrm{y}$ directions required two forces to be created and applied at two nodes each. Only forces on the $z$ direction were used for these conditions. The moment on the $z$ direction required four forces to be applied. These forces had an $x$ and a y component (see Appendix E.8 for load directions).

Table E.1 Magnitude of forces created for FEA analysis.

\begin{tabular}{|c|c|c|c|c|c|c|}
\hline Loading Case & $\mathbf{F}_{\mathbf{x}}$ & $\mathbf{F}_{\mathbf{y}}$ & $\mathbf{F}_{\mathbf{z}}$ & $\mathbf{M}_{\mathbf{x}}$ & $\mathbf{M}_{\mathbf{y}}$ & $\mathbf{M}_{\mathbf{z}}$ \\
\hline Magnitude Force [lbs.] & 31.25 & 31.25 & 62.5 & 88.39 & 88.39 & 31.25 \\
\hline
\end{tabular}

The results of the individual loading cases were analyzed to find the cases were the worst loading was found between positive and negative directions. Surprisingly, there was no difference on stress levels comparing a cases on the negative and positive directions (this may be due to the lack of holes on the model). Therefore, combined loading was determined by looking at the deformation direction of the front bottom part of the basket (critical part of the model). Loads that had adding strains were used for the combined loading. The opposite of this loading was analyzed as well to search for differences in loading. These loads were named "combined loading 1" and "combined loading 2", respectively.

\section{E. 7.1.3Boundary Conditions}

It was assumed that the pedal attachment (4 screws) could be modeled by fixing the top face of the basket with an ENCASTRE boundary condition. This boundary condition fixes all degrees of freedom of the surface it is applied on. Figure E.6 depicts this boundary condition. 


\section{E.7.2 Mesh Development}

The element type used in this analysis was an 8-node linear brick with reduced integration and hourglass control (C3D8R). The seed size was 0.075 . The determination of seed size is discussed in Appendix 7.4. There are 30190 elements C3D8R making up the model analyzed. This yields 107913 degrees of freedom. The

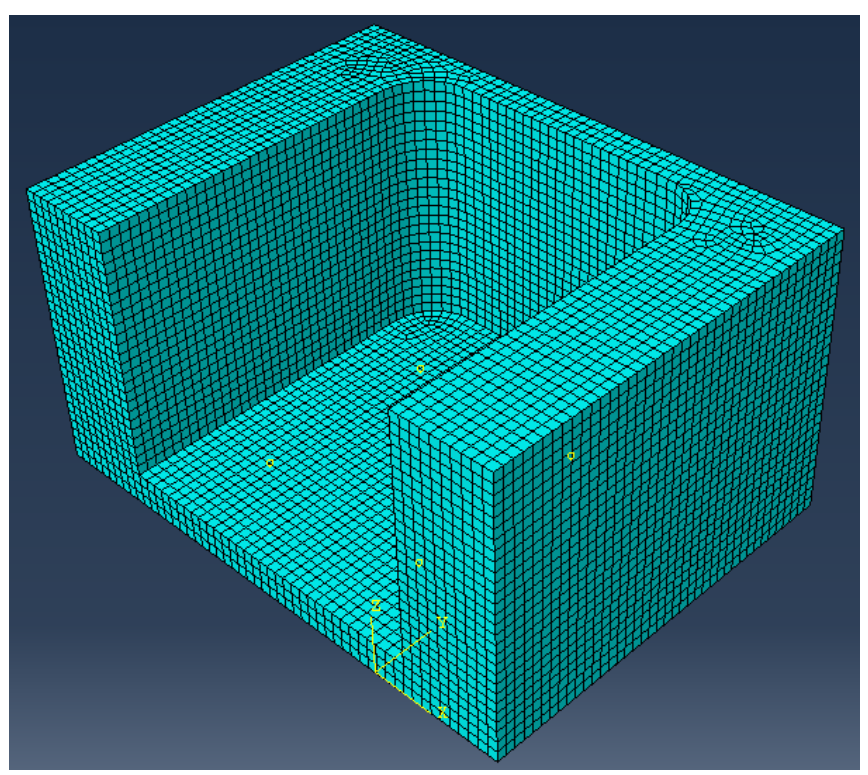

Figure E.7 Load cell housing meshed in Abaqus. quality of the elements is deemed acceptable. There were no error or warning messages on the on the Monitor option. Thus the elements meet the minimum and maximum angle and aspect ratio criteria. Figure E.7 shows the meshed model. The lack of problems is attributed to the simplicity of the model (ignoring of clearance hole features).

\section{E.7.3 Analysis}

A static general analysis was done on this analysis. This analysis assumed the forces were applied as static loads. While most of the real loading is dynamic and cyclical, static assumption is reasonable as the movement of the pedal will be based on a cycling cadence of 70 RPM and the maximum force values occur for a very short period of time. Furthermore, as the subjects mount the bike, they may put their weight on the pedal causing a static load condition. Transient start response of the cycling cadence is considerably slower than the steady state speed and thus can also be considered static. 


\section{E.7.4 Mesh Convergence}

The mesh convergence was determined by comparing the stress level on the node shown in figure E.8 with the number of degrees of freedom (DOF) in the model. The number of degrees of freedom was altered by changing the seed size. Decreasing the seed size increased the number of DOF following a power function trend.

Initially, the mesh was created very

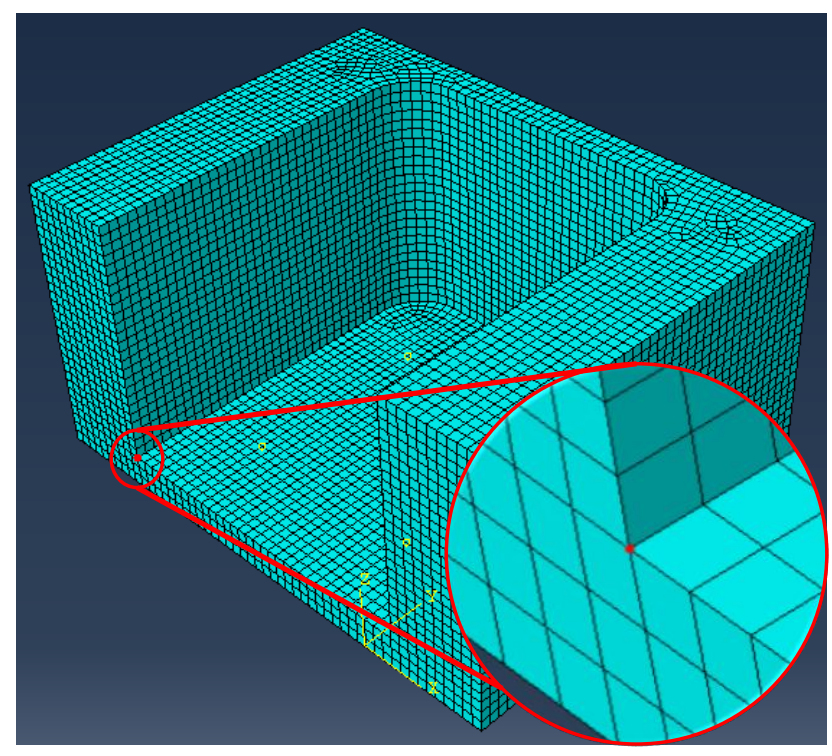

Figure E.8 Node used for mesh convergence. coarse but seed size was decreased to find the convergence of the model on a solution. The initial seed size was 0.750 while 0.050 was the smallest seed size executed. Figure E.9 displays the stress convergence with respect to the number of degrees of freedom. Although a seed size of 0.050 seems to converge better to a solution, time constrains had to be taken into account. Looking at table E.2, it can be noted that the smallest seed size takes too long to compute (over an hour of computational time required). Given the assumptions used in creating the boundary conditions, which diverge from the real attaching of the system, and the fact that there is a $20 \%$ difference in stress between the two smallest seeds but it takes over eight times the computational resources, it was decided to use the 0.075 seed size. Although the solution could be better by using a smaller mesh, the time resource could be better spent in other tasks as the boundary conditions already deviate from exact results. 
Table E.2 Variables taken into consideration when selecting seed size. Green shows selected seed size. Difference calculated with results from previous seed size.

\begin{tabular}{|c|c|c|c|c|c|c|}
\hline $\begin{array}{c}\text { Seed } \\
\text { Size }\end{array}$ & DOF & Stress & $\begin{array}{c}\text { Stress } \\
\text { Diff }\end{array}$ & \multicolumn{2}{|c|}{ Total CPU Time } & $\begin{array}{c}\text { Time } \\
\text { Diff }\end{array}$ \\
\hline & & {$[\mathrm{psi}]$} & {$[\%]$} & {$[\mathrm{s}]$} & {$[\mathrm{min}]$} & {$[\%]$} \\
\hline 0.75 & 2496 & 628.745 & $\mathrm{NA}$ & 0.7 & 0 & $\mathrm{NA}$ \\
\hline 0.5 & 3978 & 852.638 & 36 & 0.8 & 0 & 14 \\
\hline 0.25 & 16674 & 923.825 & 8 & 3 & 0.1 & 275 \\
\hline 0.125 & 100194 & 1174.41 & 27 & 39.4 & 0.7 & 1213 \\
\hline 0.1 & 207411 & 1328.71 & 13 & 127 & 2.1 & 222 \\
\hline 0.075 & 414405 & 1423.42 & 7 & 444.2 & 7.4 & 250 \\
\hline 0.05 & 1427280 & 1690.37 & 19 & 3838.3 & 64 & 764 \\
\hline
\end{tabular}

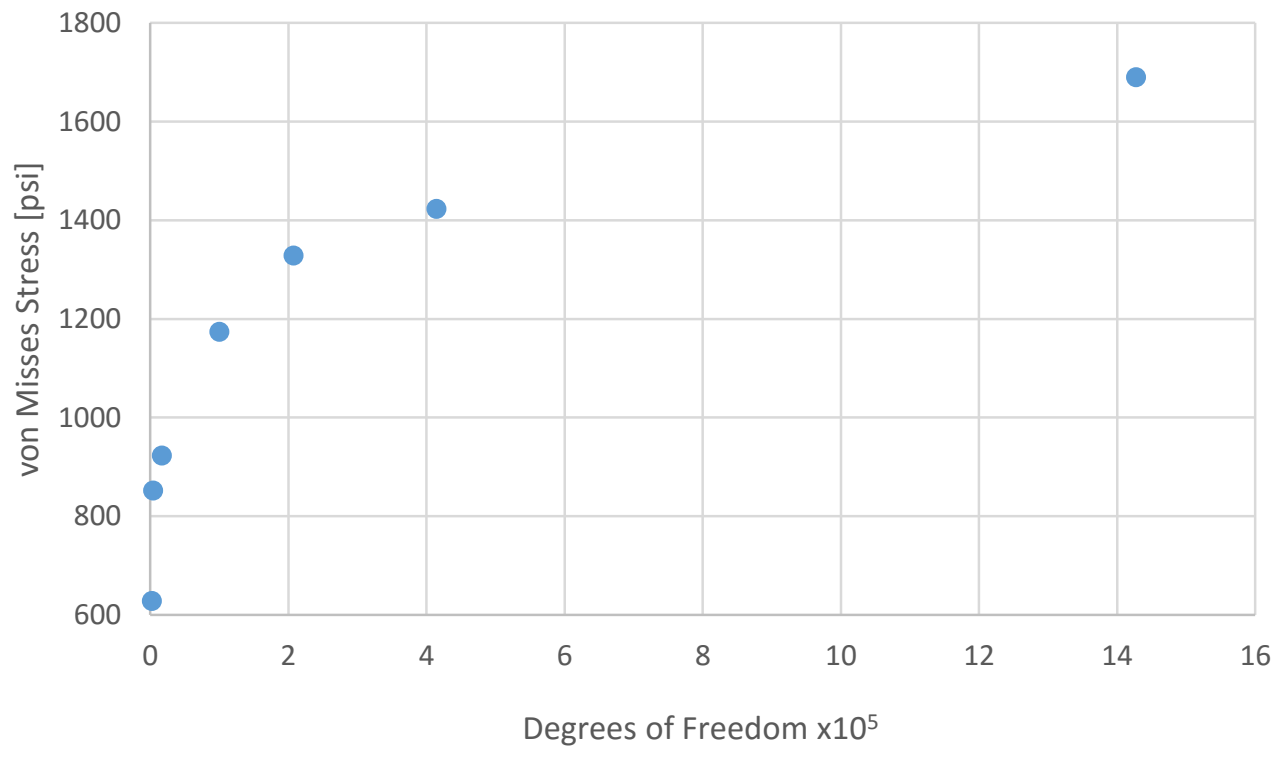

Figure E.9 Stress versus DOF. Note the last data point seems to converge best but there is a great increase in DOF. 


\section{E.7.5 Results}

The stresses were measured at two nodes in the model. One reading point was the node with the highest stress reading (labeled Node 1 on the model). The same node was used for measurements on all cases. On individual loading cases, the four loading nodes had the same stress level. For combined loading, the combination was done so strains added at node 1 . The second reading point was away from node 1 . This is done because concentrated forces are used in the loading. This creates an artificial high stress on the model that is not present on the real system. Figure E.10 shows the nodes used. Table E.3 lists the stress levels read for each loading case at the specified nodes along with the factor of safety based on a yield strength of $35 \mathrm{ksi}$ [28] and the percent difference from the expected 2.65 minimum factor of safety. Note that the combined loading 1 and 2 have the same stress levels. This symmetry may be due to the lack of holes on the model. The pedal screw hole positions are not symmetrical which would terminate the symmetry on the geometry of the model. Figure E.11 shows the result of the simulation for combined loading.

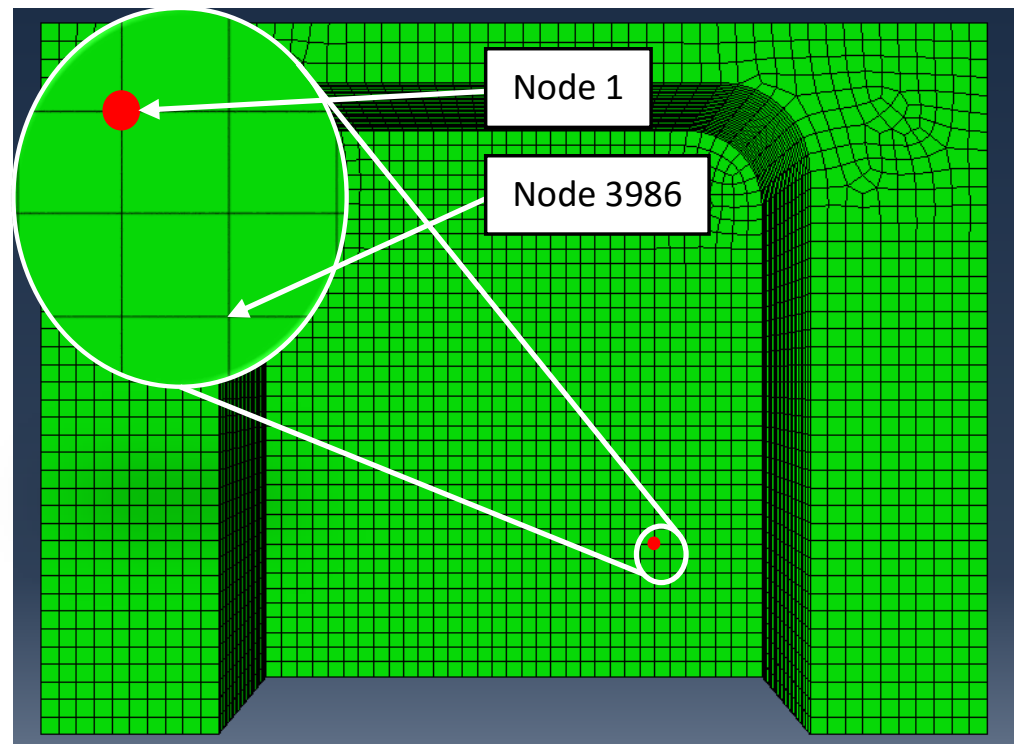

Figure E.10 Nodes used to determine stresses. 
Table E.3 Stress, safety factor, and percent difference resulting from analysis.

\begin{tabular}{|c|c|c|c|c|c|c|}
\hline & \multicolumn{3}{|c|}{ Away from Load Node } & \multicolumn{3}{c|}{ At Load Node } \\
\hline Loading & Stress & $\begin{array}{c}\text { Factor of } \\
\text { Safety }\end{array}$ & Diff & Stress & $\begin{array}{c}\text { Factor of } \\
\text { Safety }\end{array}$ & Diff \\
\hline & {$[\mathrm{psi}]$} & & {$[\%]$} & {$[\mathrm{psi}]$} & & {$[\%]$} \\
\hline $\mathrm{F}_{\mathrm{x}}$ & 1085.06 & 32.3 & 1117 & 3406.91 & 10.3 & 288 \\
\hline $\mathrm{F}_{\mathrm{y}}$ & 719.08 & 48.7 & 1737 & 3390.04 & 10.3 & 290 \\
\hline $\mathrm{F}_{z}$ & 2305.83 & 15.2 & 473 & 6490.92 & 5.4 & 103 \\
\hline $\mathrm{M}_{\mathrm{x}}$ & 3333.38 & 10.5 & 296 & 9145.8 & 3.8 & 44 \\
\hline $\mathrm{M}_{\mathrm{y}}$ & 3306.76 & 10.6 & 299 & 9077.48 & 3.9 & 45 \\
\hline $\mathrm{M}_{z}$ & 668.531 & 52.4 & 1876 & 3307.61 & 10.6 & 299 \\
\hline Combined 1 & 8840.15 & 4.0 & 49 & 24779.4 & 1.4 & -47 \\
\hline Combined 2 & 8840.15 & 4.0 & 49 & 24779.4 & 1.4 & -47 \\
\hline
\end{tabular}

S. Misses [psi]
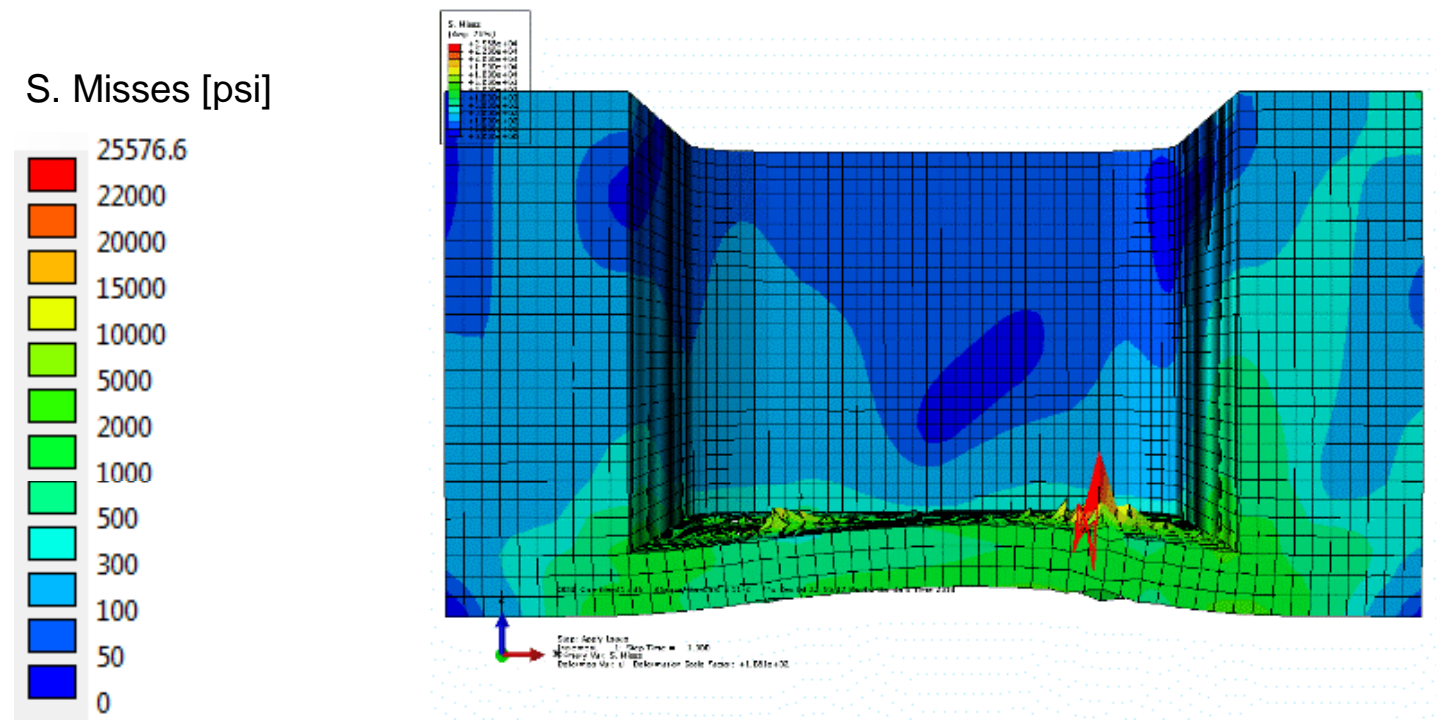

Figure E.11 Combined loading results from FEA analysis.

\section{E.7.6 Discussion}

Table E.3 shows that the factor of safety for individual loading cases are well above the expected factor of safety. At the node some distance away from the loading node the factor of safety ranges from 10.6 to 52.4 . At the loading node, the safety factor ranges from 3.8 to 10.6. The combined case, as expected has a much lower estimated factor of 
safety of 4 . This is $49 \%$ above what was expected from the rough hand calculations. This large discrepancies between results could be due to several reasons. First, the hand calculation analysis done was very rough, including only certain loads and ignoring the 3D nature of the system. The effect of the vertical walls is ignored. Thus the hand calculations, in essence, tests for a much weaker system than the one built. Next, the boundary conditions distributed the strains on the top surface decreasing the stresses on these surface. Also, lack of the holes modeled on the part does not account for stress concentrations created around these holes. Finally, the use of concentrated loads creates an artificial stress increase near the application point which is not true to the real system. Despite all these limitations on the model, the results give an estimate that suggests the baskets will handle the expected maximum loading with ease.

\section{E.7.7 Conclusions}

Custom aluminum baskets were designed to place load cells on the pedals of an upright static bicycle for biomechanics experiments. This analysis was aimed to show that the design can withstand the maximum expect loading required to take measurements on the linear regions of the load cells. The part was modeled in Abaqus using a 3D deformable body and using a static general analysis, concentrated loads simulated the loading conditions expected. Stress values were calculated and factors of safety were found. The results yielded a safety factor of 4.0, suggesting the baskets will support the expected loads. The large margin in factor of safety points to low deflections on the basket, allowing for better and more reliable measurements of the forces at the pedals during steady state cycling. 


\section{E.8 Additional Figures}

\section{E.8.1 Hand Calculations}

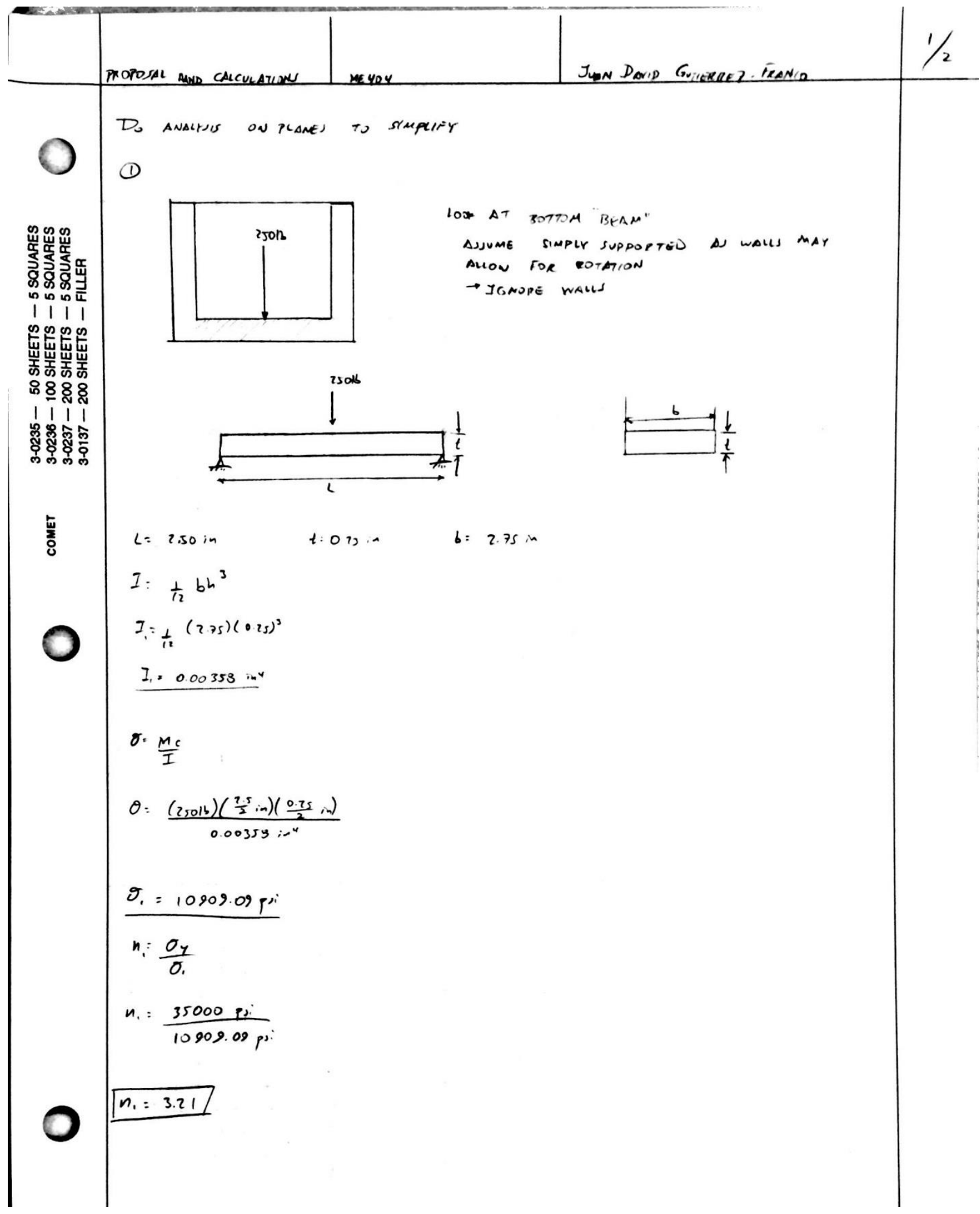




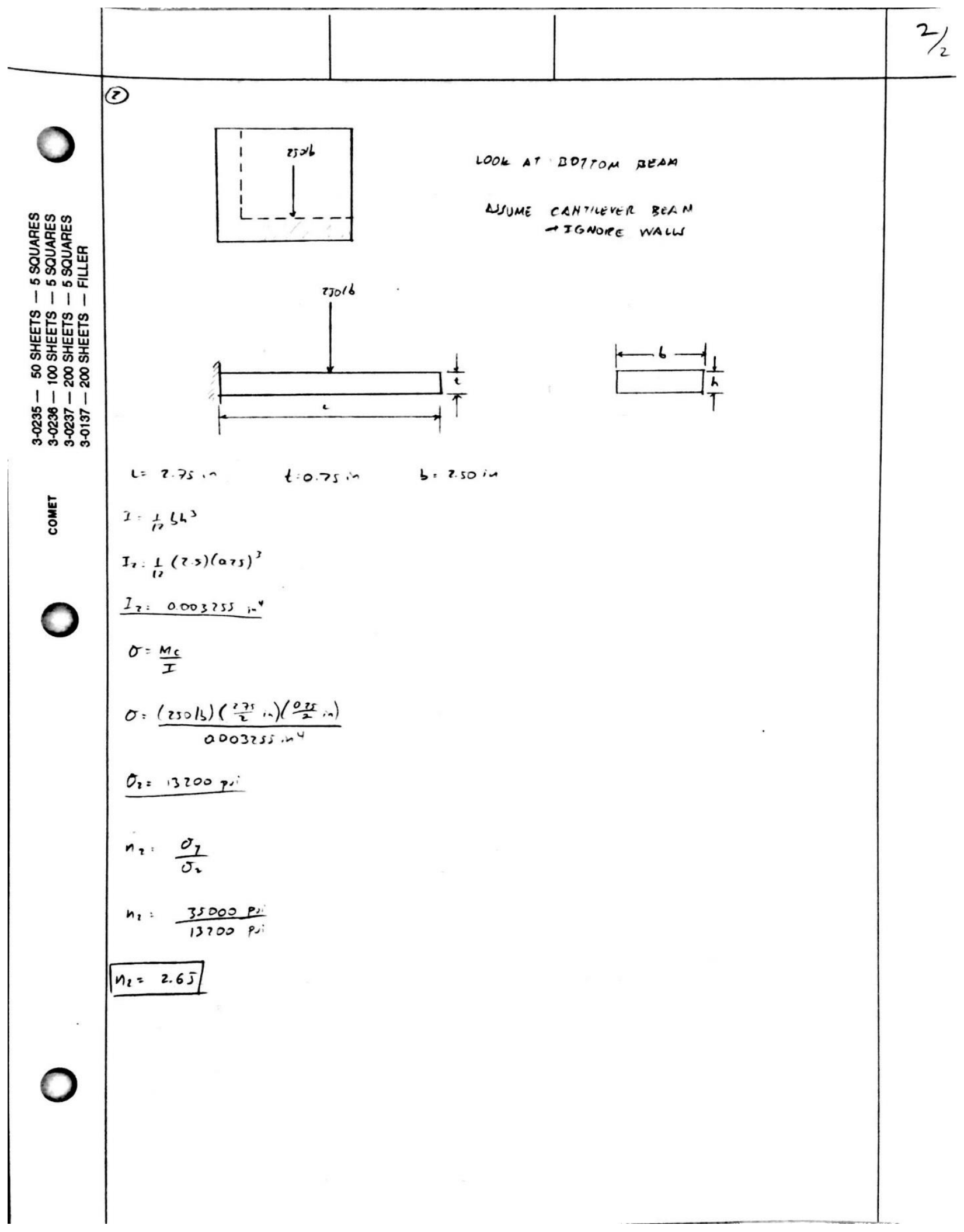




\section{E.8.2 Load Directions}

Fx
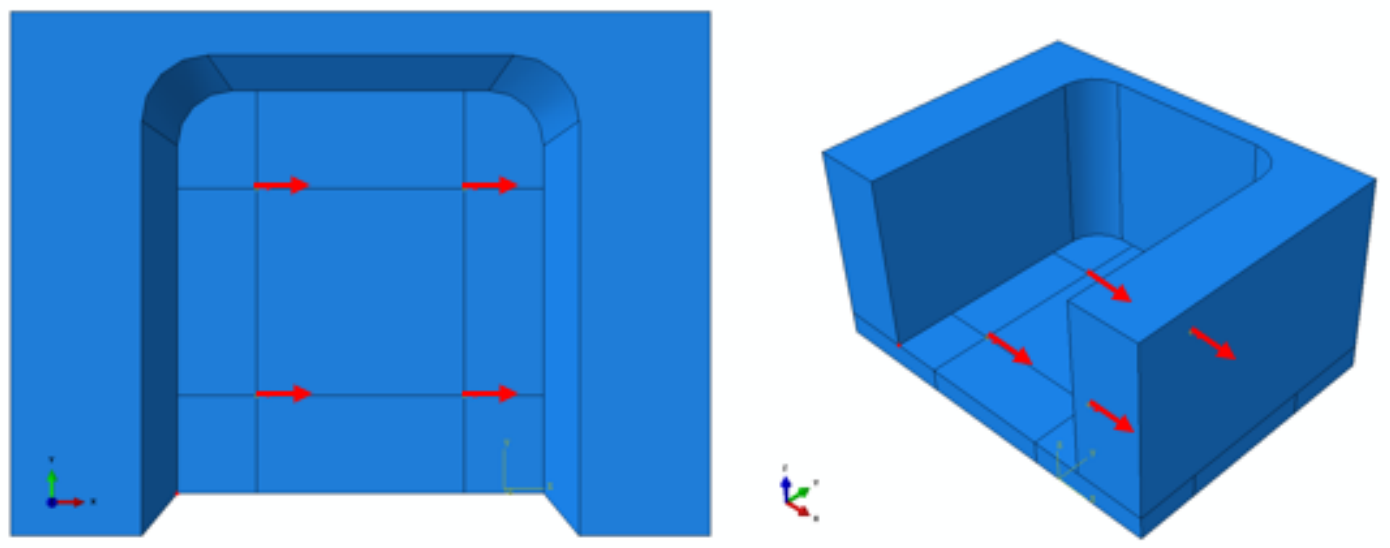

Fy
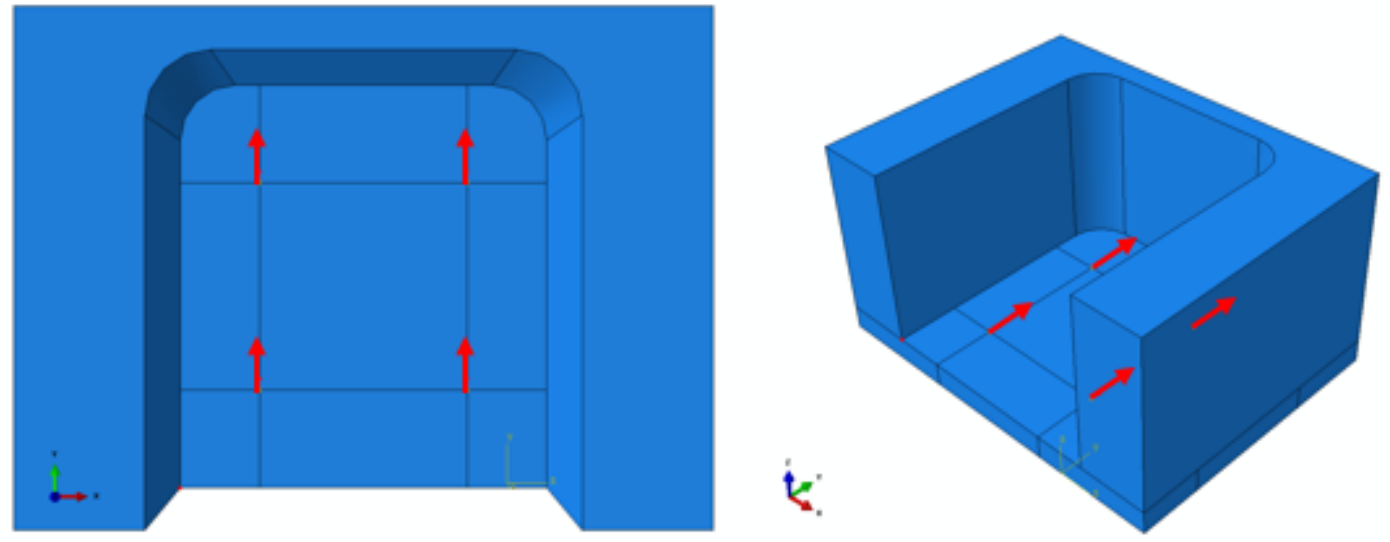

$\mathrm{Fz}$

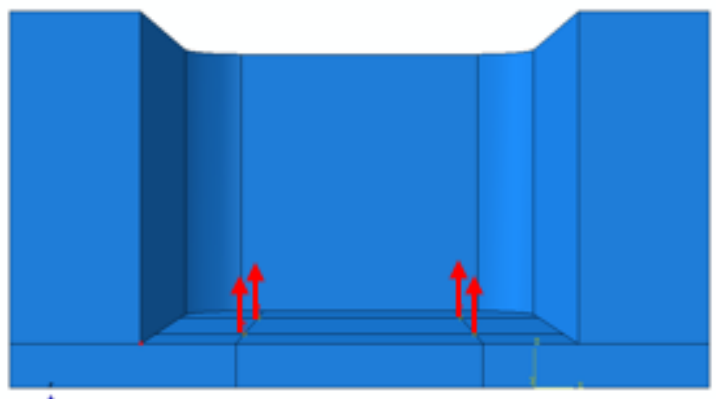

L.

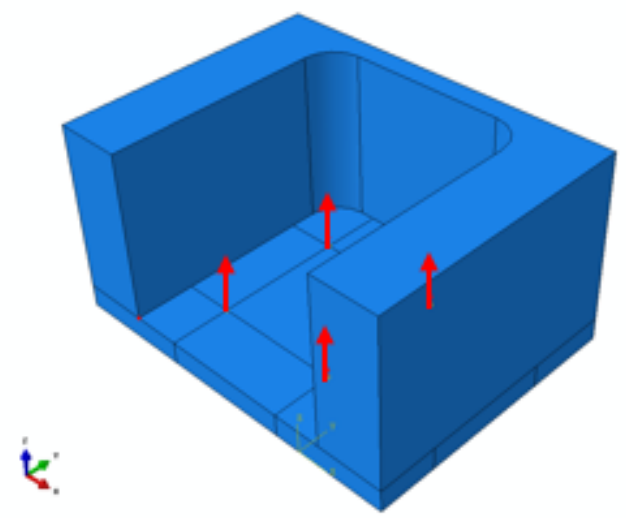




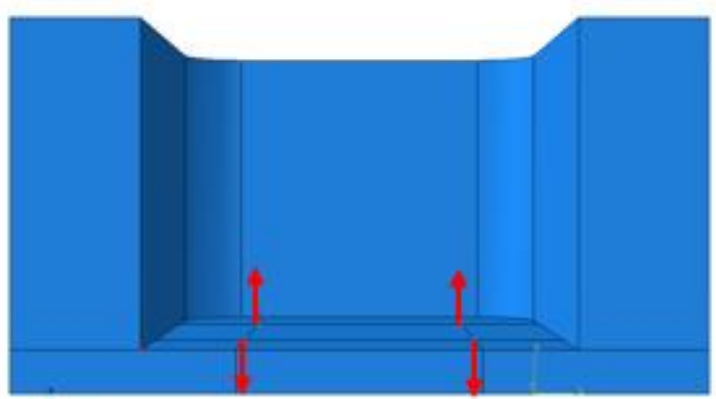

L.

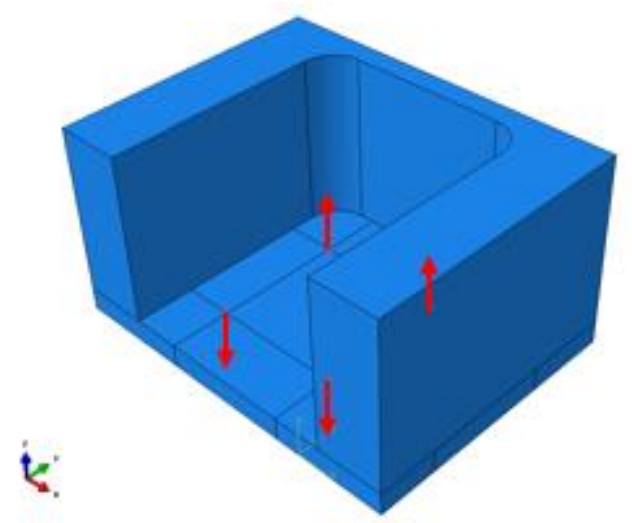

My

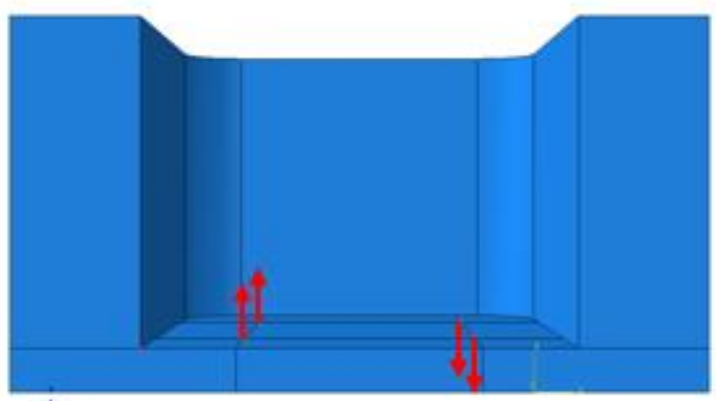

L.

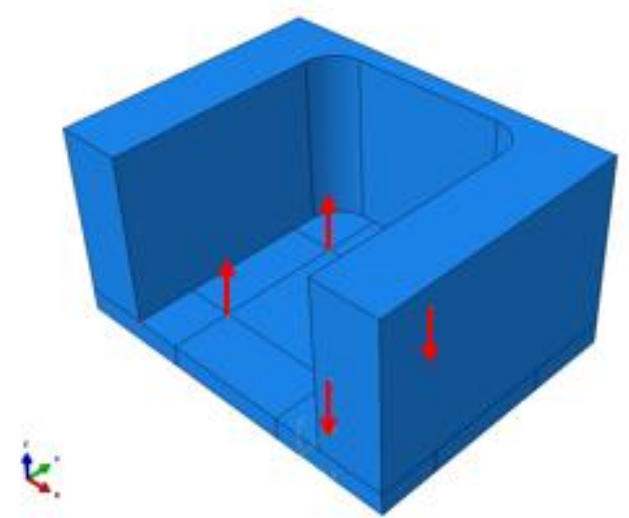

$\mathrm{Mz}$
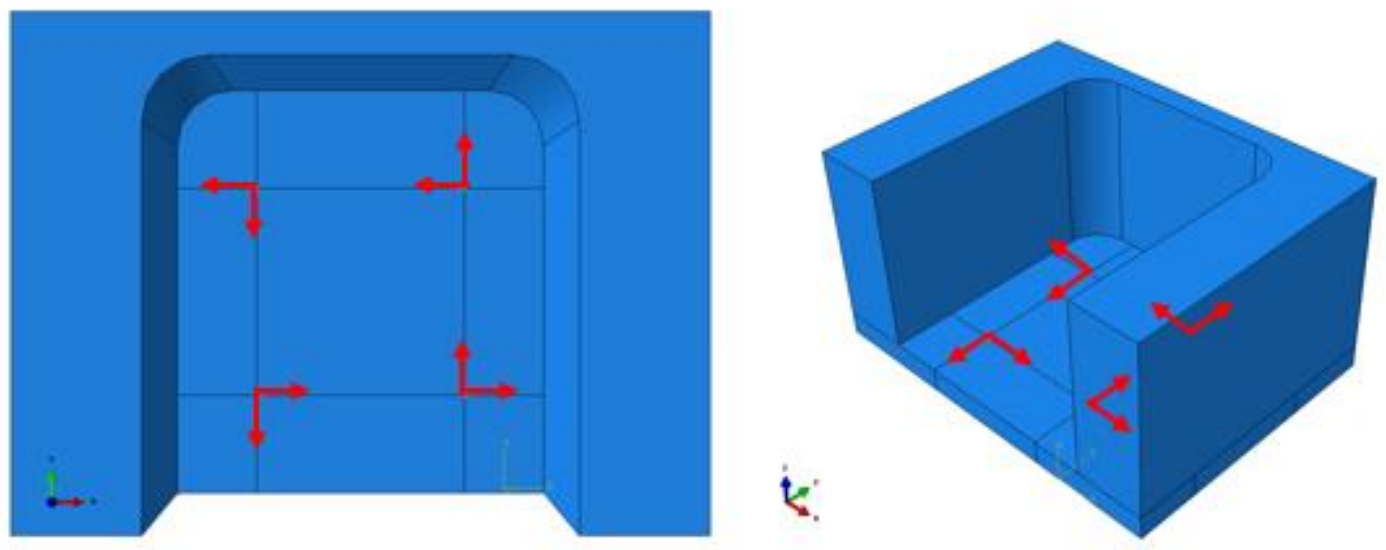


\section{E.8.3 Degrees of Freedom vs. Seed Size}

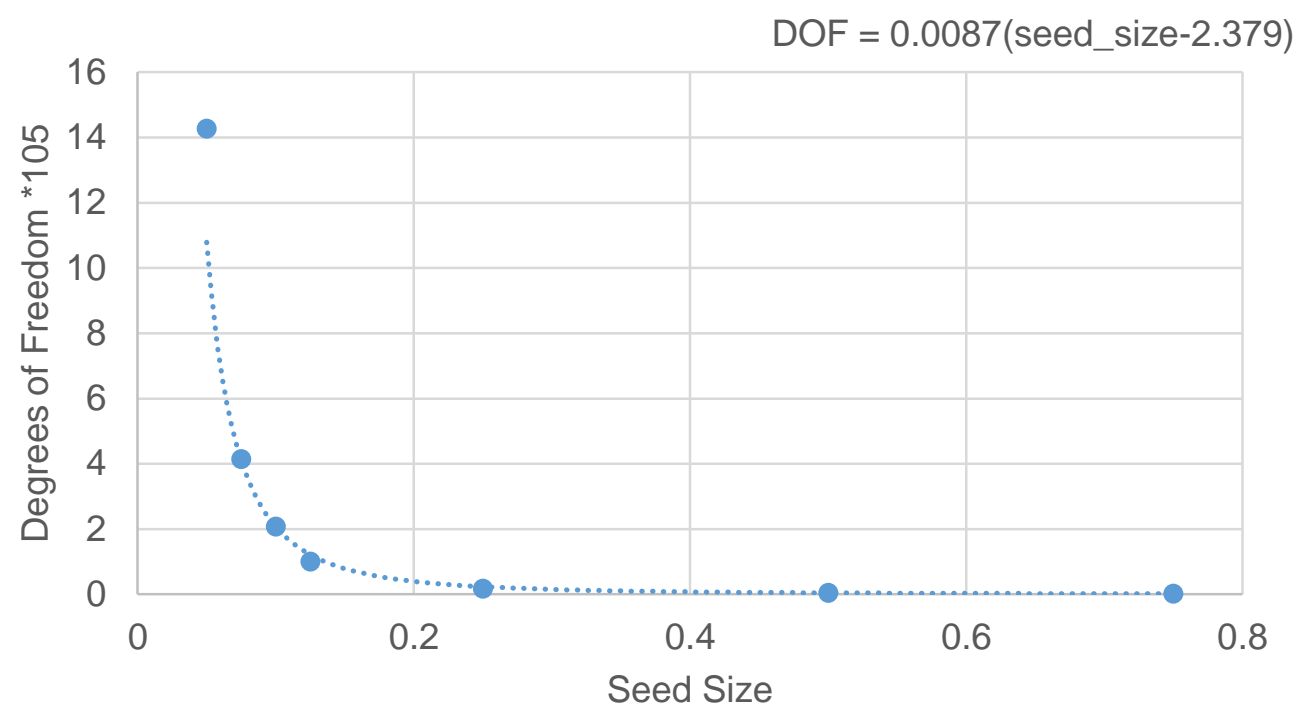




\section{E.8.4 Individual Loading Results}

\begin{tabular}{|c|c|c|}
\hline Fx & Front View & Isometric View \\
\hline+ & & \\
\hline & & \\
\hline & & \\
\hline
\end{tabular}

\begin{tabular}{|l|l|l|}
\hline Fy & Front View & Isometric View \\
\hline+ & & \\
\hline & & \\
\hline
\end{tabular}




\begin{tabular}{|l|c|c|c|}
\hline Fz & Front View & Isometric View \\
\hline+ & & & \\
\hline & &
\end{tabular}

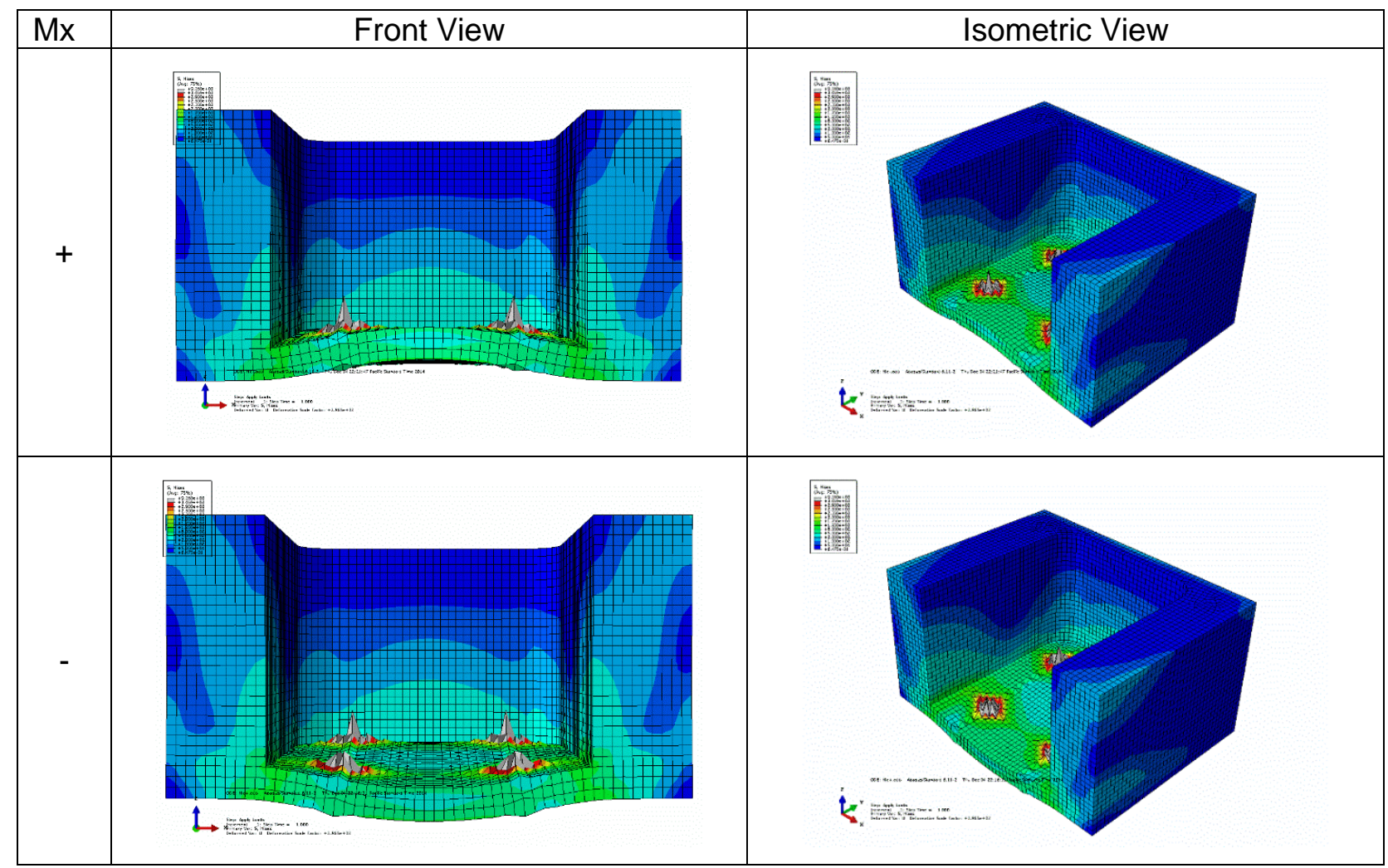




\begin{tabular}{|l|c|c|c|}
\hline My & Front View & Isometric View \\
\hline+ & & & \\
\hline
\end{tabular}

\begin{tabular}{|l|c|c|c|}
\hline $\mathrm{Mz}$ & Front View & Isometric View \\
\hline+ & & & \\
\hline
\end{tabular}

